\title{
Extremal and probabilistic problems in order types
}

\author{
Marcelo Tadeu de Sá Oliveira Sales \\ DisSERTATION SUBMITTED \\ TO THE \\ Institute of Mathematics and Statistics \\ OF THE \\ University of SÃo PAUlo \\ FOR \\ THE DEGREE \\ OF \\ MASTER OF ScIEnCES
}

Program: Mathematics

Advisor: Prof. Dr. Yoshiharu Kohayakawa

During the development of this work the author was financially supported by $\mathrm{CNPq}$

São Paulo, March 2018 


\section{Extremal and probabilistic problems in order types}

This version of the dissertation contains the corrections and alterations suggested by the members of the examining comittee during the defense of the original version of the work, realized in June 15th of 2018. A copy of the original version is available at Instituto de Matemática e Estatística da Universidade de São Paulo

Examining Comittee:

- Prof. Dr. Yoshiharu Kohayakawa (Advisor) - IME-USP

- Prof. Dr. Arnaldo Mandel - IME-USP

- Prof. Dr. Carlos Gustavo Tamm de Araújo Moreira - IMPA 


\section{Abstract}

SALES, M. T. Extremal and probabilistic problems in order types. Dissertation (Masters) - Institute of Mathematics and Statistics, University of São Paulo, São Paulo, 2018.

A configuration is a finite set of points in the plane. Two configurations have the same order type if there exists a bijection between them that preserves the orientation of every ordered triple. A configuration $A$ contains a copy of a configuration $B$ if some subset of $A$ has the same order type of $B$ and we denote this by $B \subset A$. For a configuration $B$ and a integer $N$, the extremal number

$$
\operatorname{ex}(N, B)=\max \left\{|A|: B \not \subset A \subset[N]^{2}\right\}
$$

is the maximum size of a subset of $[N]^{2}$ without a copy of $B$. We give an upper bound for general and convex cases.

A random $N$-set is a set obtained by randomly choosing $N$ points uniformly and independently in the unit square. A configuration is $n$-universal if contains all order types in general position of size $n$. We obtain the threshold for the $n$-universal property up to a $\log \log$ factor, that is, we obtain integers $N_{0}$ and $N_{1}$ with $\log \log N_{1}=O\left(\log \log N_{0}\right)$ such that if $N \gg N_{1}\left(N \ll N_{0}\right)$, then a random $N$-set is $n$-universal with probability tending to 1 (tending to 0 ). We also determine a bound for the probability of obtaining a random set without a copy of a fixed configuration.

Keywords: combinatorial geometry, probabilistic method, order types, combinatorics. 


\section{Resumo}

SALES, M. T. Problemas extremais e probabilísticos em o-tipos. Dissertação (Mestrado) - Instituto de Matemática e Estatística, Universidade de São Paulo, São Paulo, 2018.

Uma configuração é um conjunto finito de pontos no plano. Duas configurações possuem o mesmo o-tipo se existe uma bijeção entre elas que preserva a orientação de toda tripla orientada. Uma configuração $A$ contém uma cópia da configuração $B$ se algum subconjunto de $A$ possui o mesmo o-tipo que $B$ e denotamos este fato por $B \subset A$. Para uma configuração $B$ e um inteiro $N$, o número extremal

$$
\operatorname{ex}(N, B)=\max \left\{|A|: B \not \subset A \subset[N]^{2}\right\}
$$

é o maior tamanho de um subconjunto de $[N]^{2}$ sem uma cópia de $B$. Neste trabalho, determinamos cotas superiores para o caso geral e para o caso convexo.

Um $N$-conjunto aleatório é um conjunto obtido escolhendo $N$ pontos uniformemente e independentemente ao acaso do quadrado unitário. Uma configuração é $n$-universal se contém todos os o-tipos de tamanho $n$. Determinamos o limiar da propriedade de um $N$ conjunto aleatório ser $n$-universal a menos de erros da ordem de log log, isto é, determinamos inteiros $N_{0}$ e $N_{1} \operatorname{com} \log \log N_{0}=O\left(\log \log N_{1}\right)$ tais que se $N \gg N_{1}\left(N \ll N_{0}\right)$, então o $N$ conjunto aleatório é $n$-universal com probabilidade tendendo a 1 (tendendo a 0 ). Também obtivemos cotas para a probabilidade de um conjunto aleatório não possuir determinado o-tipo.

Palavras-chave: geometria combinatória, métodos probabilisticos, o-tipos, combinatória. 


\section{Contents}

1 Introduction 1

2 Preliminary Results 4

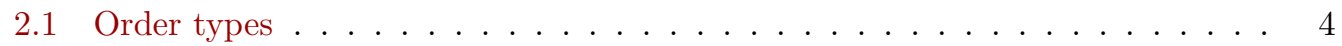

2.1.1 Probabilistic approximation . . . . . . . . . . . 7

2.1 .2 Geometric lemma . . . . . . . . . . . . . . . . . . . 10

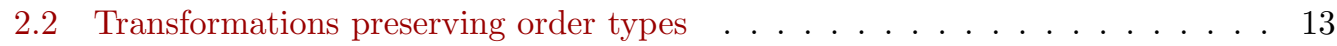

2.2.1 Affine transformation . . . . . . . . . . . . . . . . 13

2.2 .2 Projective transformation . . . . . . . . . . . . . . . . . 14

2.3 Chazelle's encoding . . . . . . . . . . . . . . . . . . . . . . . 19

2.3 .1 The lower bound . . . . . . . . . . . . . . . . 20

2.3.2 The upper bound . . . . . . . . . . . . . . . . 25

3 Extremal Results $\quad 28$

3.1 Density problem in the plane . . . . . . . . . . . . . . . . . . . . . . . 29

3.1 .1 The upper bound . . . . . . . . . . . . . . . . . 30

3.1 .2 The lower bound . . . . . . . . . . . . . . . . 32

3.1.3 A family of configuration with polynomial bounds . . . . . . . . . 33

3.2 Extremal number for the grid . . . . . . . . . . . . . . . . . 37

3.2 .1 Averaging lemma . . . . . . . . . . . . . . . . . 38

3.2 .2 Blow-up embedding . . . . . . . . . . . . . . . . . 40

3.2.3 Extremal number estimates . . . . . . . . . . . . . . . . 42

3.2 .4 Convex case . . . . . . . . . . . . . . . . . . . . . 43

4 Probabilistic Results $\quad 47$

4.1 Threshold for the $n$-universal property . . . . . . . . . . . . . . . 47

4.1.1 The probability of a given order type . . . . . . . . . . . . 48

4.1.2 Relationship between $\nu(B)$ and $\operatorname{gr}(B) \ldots \ldots \ldots$. . . . . . 51

4.1 .3 Proof of Theorem $1.3 \ldots \ldots \ldots$. . . . . . . . . . . . 52

4.2 Probability of not containing an order type . . . . . . . . . . . . 54

4.2 .1 Discretization of the probability . . . . . . . . . . . . 55

4.2 .2 Hypergraph containers . . . . . . . . . . . . . . . . 56

4.2.3 Counting theorem and proof of Theorem $1.4 \ldots 60$ 
5 Final Remarks $\quad 64$

$\begin{array}{lr}\text { A Asymptotic Estimates } & 66\end{array}$ 


\section{Chapter 1}

\section{Introduction}

Combinatorial geometry is the branch of combinatorics concerned with the study of combinatorial properties of geometric objects. Most questions in this area are intuitive easy-to-understand problems about arrangements of simple euclidean objects like points, lines and circles. For instance, what is the maximum number of incidences between $n$ points and $n$ lines? What is the minimum number of unity balls needed to cover a box of a given volume?

These questions have been studied for more than a century, mainly because of the development of combinatorics. However, only in the last decades, with the increasing development of computer technology, the area had gained great attention. Today combinatorial geometry is one of the most active and largest areas in combinatorics.

One of the most frequent objects of study in this field are finite configurations of points in the plane. By configuration we mean a set of points in the real plane. Usually, in the combinatorial context, what differs one configuration from another is not their euclidean metric properties, but instead its arrangement of points and lines in the plane. For instance, it does not matter if the diagonals of a convex quadrilateral are perpendicular or not, however it matters if the four points that we are looking at are convex or not. This leads us to the natural feeling that configurations of points should be classified by their arrangements of lines. Such a classification exists and is called the order type of a configuration.

Two configurations are said to be of the same order type if there is a bijection between them which preserves the orientation of each ordered triple. This is clearly an equivalence relation and therefore order types are equivalence classes of configurations in the plane. One can also notice that this definition is basically the same as realizable oriented matroids, which there exists a vast literature on it (see [4], [5], [27]). We also refer the reader to the recent monograph of Eppstein [8].

We shall study combinatorial aspects of order types. Past research was done in this direction. For instance, Károlyi, Solymosi and Toth [22, 23] studied order types in the context of generalizing the Erdős-Szekeres theorem [9] and Nešetřil and Valtr [25, 26] studied Ramsey-type problems. In this thesis we will focus on extremal and probabilistic problems concerning order types. 
A configuration $A$ contains a copy of order type $B$ if there exists a subset of $A$ with same order type of $B$. When there is no danger of confusion, we write $B \subset A$ to mean that order type $A$ contains a copy of order type $B$.

Given an order type $B$ of $n$ points in general position, we let

$$
\operatorname{gr}(B)=\min \left\{N \in \mathbb{N}: B \subset[N]^{2}\right\}
$$

that is, the minimum grid size $N$ such that there exists a copy with order type $B$ in $[N]^{2}$. Inspired by the graph-theoretic concept of extremal number, let $\operatorname{ex}(N, B)$ be the maximum integer $m$ such that there exists a subconfiguration of $[N]^{2}$, of size $m$, without a copy of $B$. We establish a subquadratic upper bound on $\operatorname{ex}(N, B)$ that depends only on the paramter $\operatorname{gr}(B)$.

Theorem 1.1. Let $B$ be a configuration of $n$ points in general position. Then

$$
\operatorname{ex}(N, B) \leq 4 N^{2-\eta}
$$

where $\eta=1 / 3 n \log (3 \operatorname{gr}(B))$.

In order to prove Theorem 1.1 we will study another problem of independent interest. Given a configuration $B$ of $n$ points and $\alpha>0$ a real number, let $f(B, \alpha)$ be the minimum integer $m$ such that there exists a configuration $A$ of $m$ points in the plane such that every $\alpha$ proportion of $A$ contains a copy of $B$, that is, every $X \subset A$ with $|X| \geq \alpha|A|$ contains a copy of $B$. Let $f(n, \alpha)=\max \{f(B, \alpha): B$ is in general position $\}$ be the maximum of $f(B, \alpha)$ over all order types of size $n$ in general position. We give upper and lower bounds for $f(n, \alpha)$.

Theorem 1.2. Let $\alpha \leq 1 / 2$ and $n \geq 3$. Then

$$
\frac{n^{2}}{20 \alpha \log n} \leq f(n, \alpha) \leq n^{2 n \log (1 / \alpha)} .
$$

On the probabilistic side, we will study problems related to the following random process. Given an integer $N>0$, we construct a set $U \subset[0,1]^{2}$ by randomly choosing $N$ points in the unit square uniformly and independently. We will often call $U$ a random $N$-set. This random process is well known and was already used in other combinatorial problems (see for instance [7]). The problem of computing the probability that $U$ has a certain order type is not well understood. Valtr [34] computed the probability of a random set being convex, but we do not know much more about the other cases. One of our goals is to provide bounds for general order types.

Given an integer $n>0$, a configuration $A$ is $n$-universal if $A$ contains a copy of every order type of size $n$ in general position. Our first probabilistic result gives the threshold for the $n$-universal property up to a $\log \log$ factor. 
Theorem 1.3. There exist positive real numbers $c_{1}$ and $c_{2}$ such that the following holds. Let $N>0$ be an integer and $U$ a random $N$-set. Then,

$$
\lim _{n \rightarrow \infty} \mathbb{P}(U \text { is n-universal })= \begin{cases}1, & \text { if } N \geq 2^{2^{c_{1} n}} \\ 0, & \text { if } N \leq 2^{2^{c_{2} n}} .\end{cases}
$$

The second result is a superexponential upper bound for the probability of obtaining a random set without a copy of a fixed order type.

Theorem 1.4. For every configuration $B$, there exists a number $c:=c(B)$ such that for every sufficiently large $N$ the following holds. If $U$ is an $N$-random set, then

$$
\mathbb{P}(B \not \subset U) \leq\left(\frac{1}{N}\right)^{c N} .
$$

This thesis is organized in the following way. In Chapter 2 we discuss preliminary results on order types that will be helpful for our work. Section 2.1 contains results on the space of realizations of order types. Some of these results are original and fundamental to the rest of the work. Section 2.2 introduces some transformations in the real plane that preserve order types and Section 2.3 solves Chazelle's encoding problem, that is, the problem of finding the minimum grid that contains all order types of a given size.

Chapter 3 is devoted to solving the two extremal problems presented above. Section 3.1 is on the study of the density problem in the plane (Theorem 1.2) and Section 3.2 is about the study of the density problem in the grid (Theorem 1.1). In Chapter 4 we study the probabilistic model presented in this introduction. Bounds for the probability of a general order type and Theorem 1.3 are given in Section 4.1. The proof of Theorem 1.4 is presented in Section 4.2. Throughout the work, we do not try to optimize the constants. 


\section{Chapter 2}

\section{Preliminary Results}

In this chapter we will introduce some important concepts for our work. Denote by $[n]$ the set of integers $\{1,2, \ldots, n\}$. Given two functions $f, g: \mathbb{R} \rightarrow \mathbb{R}_{+}$, we say that $f(x)=$ $O(g(x))$ if and only if there exist some constants $C>0$ and $x_{0}$ such that $f(x) \leq C g(x)$ for every $x \geq x_{0}$. Also we say that $f(x)=o(g(x))$ if and only if for every $\varepsilon>0$ there exists a $x_{0}$ such that $f(x) \leq \varepsilon g(x)$ for every $x \geq x_{0}$. When there exist constants $c_{1}$ and $c_{2}$ and $x_{0}$ such that $c_{1} g(x) \leq f(x) \leq c_{2} g(x)$ we say that $f(x)=\Theta(g(x))$. Now we proceed to introduce basic properties about order types.

\section{$2.1 \quad$ Order types}

Given three points $x=\left(x_{1}, x_{2}\right), y=\left(y_{1}, y_{2}\right), z=\left(z_{1}, z_{2}\right) \in \mathbb{R}^{2}$ let

$$
[x y z]=\frac{1}{2}\left|\begin{array}{lll}
x_{1} & x_{2} & 1 \\
y_{1} & y_{2} & 1 \\
z_{1} & z_{2} & 1
\end{array}\right| .
$$

be the signed area of the triangle $x y z$. Also let

$$
\operatorname{sgn} x= \begin{cases}-, & \text { if } x<0 \\ 0, & \text { if } x=0 \\ +, & \text { if } x>0\end{cases}
$$

be the sign function of the real numbers.

Define $\chi:\left(\mathbb{R}^{2}\right)^{3} \rightarrow\{-, 0,+\}$ as a function that sends an ordered triple of points in the cartesian plane to the sign of their corresponding signed area. In other words, for an oriented triple $(x, y, z) \in\left(\mathbb{R}^{2}\right)^{3}$ we have

$$
\chi(x, y, z)=\operatorname{sgn}[x y z]
$$

Definition 2.1. A configuration of $n$ points in the plane is an $n$-subset $A \subset \mathbb{R}^{2}$. We say 
that two configurations $A$ and $B$ have the same order type, and denote by $A \cong B$ ( $A$ is isomorphic to $B$ ), if there exist a bijection $\iota: A \rightarrow B$ such that

$$
\chi(x, y, z)=\chi(\iota(x), \iota(y), \iota(z)), \quad \forall x, y, z \in A,
$$

i.e., if ८ preserves the orientation of every ordered triple in $A^{3}$.

Definition 2.1 says that having the same order type is an equivalence relation and therefore one can partition the set of configurations into equivalence classes. We call the equivalence class of a configuration $A$ as the order type of $A$. Notice that Definition 2.1 allows configurations not in general position, i.e., configurations with three or more collinear points. So, there exists order types not in general position.

A more geometric approach for defining order types can be given as follows: Consider the directed straight line $\overrightarrow{x y}$ passing through the points $x$ and $y$, in this order. This straight line divides the plane into two open half-planes, $H^{+}$and $\mathrm{H}^{-}$, the half-planes on the left and right side of the straight line, respectively. A simple application of linear algebra gives us

$$
\chi(x, y, z)= \begin{cases}+, & \text { if } z \in H^{+} \\ 0, & \text { if } z \in \overrightarrow{x y} \\ -, & \text { if } z \in H^{-}\end{cases}
$$

Given a configuration $A$ of $n$ points, one can uniquely characterizes its order type by counting the number of points on the left or right side of every straight line determined by $A$. More precisely, let $M:=M[A]=\left(m_{i j}\right)$ be the matrix of entries in $A \times A$ such that $m_{i j}$ is the number of points on the left side of the directed straight line $\overrightarrow{i j}$ for $i \neq j$ and $m_{i j}=-1$ for $i=j$. If two configurations $A$ and $B$ are isomorphic, then there exists bijection $\iota: A \rightarrow B$ such that $M[A]=M[\iota(A)]$. In other words, if two configurations have the same order type, then there exists a bijection which preserves the number of points on each side of a line. The converse is also true.

Proposition 2.2 ([14], Theorem 1.8). Two configurations $A$ and $B$ have the same order type if and only if there exists a bijection $\iota: A \rightarrow B$ such that $M[A]=M[\iota(A)]$.

This approach has the advantage of connecting the definition of order types with the notion of classifying configurations by its arrangements of points and lines, which from a practical point of view, makes it easier to identify different order types. For instance, consider the configurations in Figure 2.1. A convex quadrilateral and a triangle with an interior point. Although these two configurations have the same number of points, they do not have the same order type. One can see that by noticing that the convex quadrilateral has only two lines that divides the configuration in one point to the left and one to the right (dashed lines) and a triangle with an interior point contains three such lines. Then by Proposition 2.2 they have different order types. We can also see, by the same proposition, that all convex $n$-gon have the same order type and we call this equivalence class as the convex order type. 

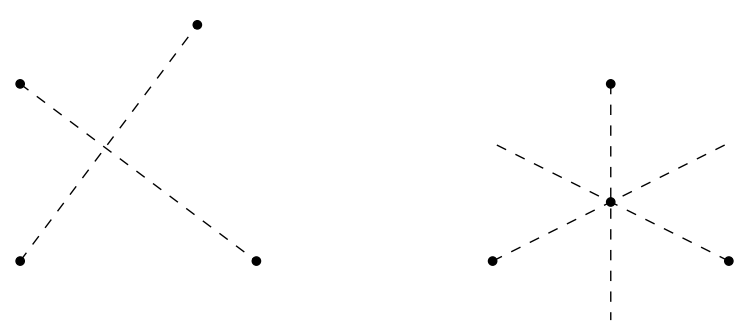

Figure 2.1: Two configurations of size 4 .

A very natural questions is to ask the number of distinct order types of size $n$. A first try would be to use the natural estimation given by Definition 2.1. Since an order type is uniquely determined by the orientation of ordered triples and there are only 3 choices for every ordered triple, we obtain there are at most $3^{\left(\begin{array}{c}n \\ 3\end{array}\right)}$ distinct order types. A suprising result is that this estimation is actually very far from the true. Goodman and Pollack [15] showed that the number of distinct order types is almost exponential on $n$.

Theorem 2.3 ([15]). There are $2^{4 n \log n+O(n)}$ distinct order types of size $n$.

In general, we will represent order types by their elements and consider any relation between order types by its representatives. Thus for configurations $A$ and $B$, we say that $A$ contains a copy of $B$ if $A$ contains an actual subset $X$ of the same order type of $B$. When there is no danger of confusion, we denote this relation by $B \subset A$. For example, a configuration $B$ contains a copy of a convex 5 -gon if there exists a subset of $B$ that it is isomorphic to a convex 5 -gon.

For a configuration $B$ of size $n$, let

$$
\Gamma[B]=\left\{A \in\left(\mathbb{R}^{2}\right)^{n}: A \cong B\right\}
$$

be the set of ordered $n$-tuples in $\mathbb{R}^{2}$ isomorphic to $B$, i.e., the set of realizations of the order type of $B$ in $\left(\mathbb{R}^{2}\right)^{n}$. Here we abuse the notation and identify an ordered $n$-tuple with its underlying configuration. That is, an ordered $n$-tuple $A=\left(a_{1}, \ldots, a_{n}\right)$ is isomorphic to $B$ if the underlying set $\left\{a_{1}, \ldots, a_{n}\right\}$ is isomorphic to $B$.

Given an ordered $n$-tuple $A=\left(a_{1}, \ldots, a_{n}\right)$ in $\left(\mathbb{R}^{2}\right)^{n}$ we can define the vector $\chi_{A}:\left(\begin{array}{c}{[n]} \\ 3\end{array}\right) \rightarrow$ $\{-, 0,+\}$ as

$$
\chi_{A}(i, j, k)=\chi\left(a_{i}, a_{j}, a_{k}\right), \quad \forall 1 \leq i<j<k \leq n .
$$

This definition allows us to characterize an order type by its possible labelings. In fact, for a configuration $B$, let $B_{1}, \ldots, B_{n}$ ! be all the possible ordering of its points. Then the order type of a configuration $B$ can be characterized by the vectors $\chi_{B_{i}}$, for $1 \leq i \leq n$ !. That is, if a ordered $n$-tuple $A$ is such that $\chi_{A}=\chi_{B_{i}}$ for some $i$, then $A \cong B$.

Although all definitions so far include configurations not in general position, in the text we will work almost only with order types in general position. The main observation of this section is that the set of realizations of an order type in general position is open. 
Proposition 2.4. Let $B$ be a configuration of $n$ points in general position. Then $\Gamma[B]$ is open in $\mathbb{R}^{2 n}$.

Proof. Let $\chi_{1}, \ldots, \chi_{m}$ be the distinct vectors for all possible orderings of $B$, with $m \leq n$ ! (Although we have $n$ ! possible orderings, some of them may generate the same vector). For an ordered configuration $A=\left(a_{1}, \ldots, a_{n}\right) \in\left(\mathbb{R}^{2}\right)^{n}$ we have that $A \cong B$ if and only if there exists $t \in[m]$ such that $\chi_{A}=\chi_{i}$. It follows that

$$
\Gamma[B]=\bigcup_{t=0}^{m}\left\{A \in\left(\mathbb{R}^{2}\right)^{n}: \chi_{A}=\chi_{t}\right\} .
$$

Thus if we prove that $\left\{A \in\left(\mathbb{R}^{2}\right)^{n}: \chi_{A}=\chi_{t}\right\}$ is open for every $1 \leq t \leq m$, then we are done.

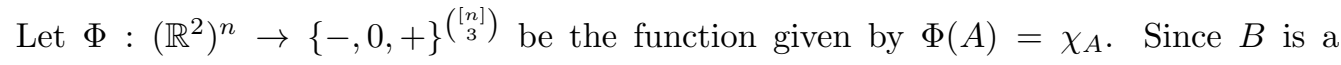
configuration in general position, it follows that $\chi_{t}(i, j, k)=+$ or $\chi_{t}(i, j, k)=-$ for dis-

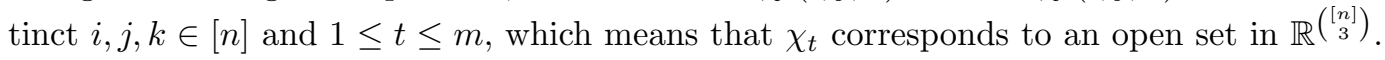
Thus $\left\{A \in\left(\mathbb{R}^{2}\right)^{n}: \chi_{A}=\chi_{t}\right\}=\Phi^{-1}\left(\chi_{t}\right)$ is the preimage of an open set. Since $\chi$ is, by definition, a polynomial with 6 variables, we obtain that $\chi$ is continuous and therefore $\Phi$ is also continuous. Finally, using that preimage of continuous function in a open set is open, we obtain that $\left\{A \in\left(\mathbb{R}^{2}\right)^{n}: \chi_{A}=\chi_{t}\right\}$ is open.

Next we explore two related results: The first one shows that we can approximate the probability of obtaining an order type in a continuous set by the probability of obtaining it in a discrete set. The second one uses geometric properties to compute how much we can perturbate a configuration $B$ without changing its order type.

\subsubsection{Probabilistic approximation}

In this subsection we study how to approximate the probability of an order type in a unit square by its probability in a grid. Consider the process described in the introduction, i.e., a $n$-random set $U$ is a set obtained by choosing randomly and independently $n$ points in $[0,1]^{2}$. The same process could be discretized by replacing the unit square by the $[m]^{2}$ : For every integer $m$ let $V_{m}$ be the $n$-random set obtained by choosing uniformly and independently $n$ points in the grid $[m]^{2}$. The next lemma shows that we can compute probabilities in $U$ by $V_{m}$.

Lemma 2.5. Let $U$ be an $n$-random set in $[0,1]^{2}$ and for every integer $m$, let $V_{m}$ be an $n$-random set in $[m]^{2}$. Then for any configuration $B$ of $n$ points, the following equality holds

$$
\mathbb{P}(U \cong B)=\lim _{m \rightarrow \infty} \mathbb{P}\left(V_{m} \cong B\right)
$$

A remark is that $\mathbb{P}(U \cong B)$ always exists. In fact, $\mathbb{P}(U \cong B)=\int_{[0,1]^{2 n}} \mathbb{1}_{\mathcal{B}} \mathrm{d} \mu=\mu(\mathcal{B})$, where $\mathcal{B}=\left([0,1]^{2}\right)^{n} \cap \Gamma[B]$ is all the possible ordered $n$-tuples isomorphic to $B$ in the unit square and $\mu$ is the Lebesgue measure defined in $\mathbb{R}^{2 n}$. There are two possible cases: If $B$ is in general position, then by Proposition 2.4 the set $\mathcal{B}$ is an intersection of a open and a 
closed set, therefore is Lebesgue measurable. If $B$ is not in general position, one can show (Corollary 2.7) that $\mathcal{B}$ is a subset of a set of measure 0 and is also Lebesgue measurable. This proves the existence of the probability. Our intention is to show more, that $\mathcal{B}$ is measurable in terms of Riemann integrals.

We will follow the approach in [31]. Given a bounded set $C$ let $R$ be a rectangle such that $C \subset R$. The next theorem is a well-known result in multidimensional real analysis.

Theorem 2.6 ([31], Theorem 3.9). The function $\mathbb{1}_{C}: R \rightarrow \mathbb{R}$ is Riemann integrable if and only if the boundary of $C$ has measure 0 .

Theorem 2.6 gives us a characterization of integrable indicator functions by the measure of the boundary of its set. Fortunately, we know how to calculate the measure of $\partial \mathcal{B}$.

Corollary 2.7. Let $B$ be a configuration with $n$ points, not necessarily in general position, and let $\mathcal{B}=\left([0,1]^{2}\right)^{n} \cap \Gamma[B]$. Then the indicator function $\mathbb{1}_{\mathcal{B}}:\left([0,1]^{2}\right)^{n} \rightarrow \mathbb{R}$ is Riemann integrable.

Proof. Let $\mathcal{C}$ be the set of all configuration of size $n$ not in general position inside $[0,1]^{2}$. We claim that $\mathcal{C}$ has measure 0 . By the previous observation, we know that $\mu(\mathcal{C})=$ $\mathbb{P}(U \in \mathcal{C})$ for an $n$-random set $U$. Given a triple $\{x, y, z\} \in\left(\begin{array}{l}U \\ 3\end{array}\right)$, let $E_{x, y, z}$ be the event that $x, y, z$ are collinear. Since a straight line has measure 0 in the euclidean plane, we obtain that $\mathbb{P}\left(E_{x, y, z}\right)=0$ for every $\{x, y, z\} \in\left(\begin{array}{c}U \\ 3\end{array}\right)$. Therefore, an union bound argument shows that

$$
\mu(\mathcal{C})=\mathbb{P}(U \in \mathbb{C}) \leq \sum_{\{x, y, z\} \in\left(\begin{array}{c}
U \\
3
\end{array}\right)} \mathbb{P}\left(E_{x, y, z}\right)=0
$$

If $B$ is not in general position, then $\mathcal{B} \subset \mathcal{C}$. Therefore, $\mu(\mathcal{B}) \leq \mu(\mathcal{C})=0$ and $\mathbb{1}_{\mathcal{B}}$ is Riemann integrable by Theorem 2.6, since $\mu(\partial \mathcal{B}) \leq \mu(\mathcal{B})=0$. Now if $B$ is in general position, note that $\partial \mathcal{B}$ is contained in the union of the borders of $\Gamma[B]$ and $\left([0,1]^{2}\right)^{n}$. It is easy to show that $\mu\left(\partial\left([0,1]^{2}\right)^{n}\right)=0$. In the proof of Proposition 2.4 we proved that $\Gamma[B]$ is a union of preimages of open sets of a continuous polynomials $\Phi$. Those preimages are of the form $\mathcal{O}=\left\{A \in\left(\mathbb{R}^{2}\right)^{n}: \chi_{A}=\chi_{C}\right\}=\Phi^{-1}\left(\chi_{C}\right)$ for some ordered $n$-tuple $C$ isomorphic to $B$. Since preimage of a closed set of a continuous function is closed, we obtain that $\partial \mathcal{O}$ is the preimage of the border of the open set defined by the image of $\chi_{C}$. However the border of the image of $\chi_{C}$ corresponds to the image of configurations $D$ such that for some $i, j, k$, we have $\chi_{D}(i, j, k)=0$. That is, the preimage of the border corresponds to a union of order types not in general position, which implies that $\partial \mathcal{O} \subset \mathcal{C}$. Since $\Gamma[B]$ is a union of such $\mathcal{O}$ 's, it follows that $\mu(\partial \Gamma[B])=0$ and $\mu(\mathcal{B})=0$.

A partition of $[0,1]^{d}$ is a collection $P=\left(P_{1}, \ldots, P_{d}\right)$, where each $P_{i}$ is a partition of intervals of $[0,1]$. Let $P_{i}$ partitions $[0,1]$ into $0=a_{0, i}<a_{1, i}<\cdots<a_{t_{i}, i}=1$. Thus $P$ partitions $[0,1]^{d}$ into $t_{1} t_{2} \ldots t_{d}$ rectangles $P_{I}$ of the form $\left[a_{i_{1}-1,1}, a_{i_{1}, 1}\right] \times \cdots \times\left[a_{i_{d}-1, d}, a_{i_{d}, d}\right]$. Define the oscillation of a function $f:[0,1]^{d} \rightarrow \mathbb{R}$ in a rectangle $B$ as

$$
\omega(f, B)=\sup _{x \in B} f(x)-\inf _{x \in B} f(x) .
$$


One characterization given in [31] of $f$ being Riemann integrable is that for every $\varepsilon>0$ there exists a partition $P$ such that

$$
\sum_{I \in\left[t_{1}\right] \times \cdots \times\left[t_{d}\right]} \omega\left(f, P_{I}\right) \mu\left(P_{I}\right)<\varepsilon
$$

Given a rectangle $B$, let

$$
\operatorname{diam}(B)=\sup _{x, y \in B}\|x-y\|
$$

be the diameter of $B$. The next theorem is a technical result that shows that the last characterization holds for every partition with sufficiently small rectangles.

Theorem 2.8. Let $f:[0,1]^{d} \rightarrow \mathbb{R}$ be a Riemann integrable function and $\varepsilon>0$. Then there exists $\delta>0$ such that any partition $P$ of $[0,1]^{d}$ with $\operatorname{diam}\left(P_{I}\right)<\delta$, for every $I \in$ $\left[t_{1}\right] \times \cdots \times\left[t_{d}\right]$, satisfies

$$
\sum_{I \in\left[t_{1}\right] \times \cdots \times\left[t_{d}\right]} \omega\left(f, P_{I}\right) \mu\left(P_{I}\right)<\varepsilon .
$$

Proof. Since $f$ is Riemann integrable, there exists a partition $Q$ such that

$$
\sum_{J \in\left[r_{1}\right] \times \cdots \times\left[r_{d}\right]} \omega\left(f, Q_{J}\right) \mu\left(Q_{J}\right)<\frac{\varepsilon}{2} .
$$

Consider any partition $P$ of $[0,1]^{d}$ such that $\operatorname{diam}\left(P_{I}\right)<\delta$. Fixed a rectangle $P_{I}$ of $P$ there are two possibilities: Either there exists a rectangle $Q_{J}$ of $Q$ such that $P_{I} \subset Q_{J}$, or $P_{I}$ intersects two or more rectangles of $Q$. In the first case, by the definition of oscillation, we obtain $\omega\left(f, P_{I}\right) \leq \omega\left(f, Q_{J}\right)$. In the second case, we use that $\omega\left(f, P_{I}\right) \leq 2 M$, where $M=$ $\sup _{x \in[0,1]^{d}}|f(x)|$. Let $\mathcal{P}_{1}$ be the collection of rectangles satisfying the first case, and let $\mathcal{P}_{2}$ be the collection of rectangles satisfying the second case. Thus,

$$
\begin{aligned}
\sum_{I \in\left[t_{1}\right] \times \cdots \times\left[t_{d}\right]} \omega\left(f, P_{I}\right) \mu\left(P_{I}\right) & \leq \sum_{P_{I} \in \mathcal{P}_{1}} \omega\left(f, Q_{J}\right) \mu\left(P_{I}\right)+\sum_{P_{I} \in \mathcal{P}_{2}} 2 M \mu\left(P_{I}\right) \\
& \leq \sum_{P_{I} \in \mathcal{P}_{1}} \omega\left(f, Q_{J}\right) \mu\left(Q_{J}\right)+2 M \sum_{P_{I} \in \mathcal{P}_{2}} \mu\left(P_{I}\right) \\
& <\frac{\varepsilon}{2}+2 M \sum_{P_{I} \in \mathcal{P}_{2}} \mu\left(P_{I}\right) .
\end{aligned}
$$

Therefore we only need to estimate the volume of all rectangles in $\mathcal{P}_{2}$.

Write $Q=\left(Q_{1}, \ldots, Q_{d}\right)$ where $Q_{i}$ partitions $[0,1]$ into $0=b_{0, i}<b_{1, i}<\cdots<b_{r_{i}, i}=1$. Every rectangle in $\mathcal{P}_{2}$ intersects at least one hyperplane of the form $[0,1] \times \cdots \times\left\{b_{j, i}\right\} \times$ $\cdots \times[0,1]$. Then it makes sense to estimate the volume of all rectangles intersecting such a hyperplane. Since $\operatorname{diam}\left(P_{I}\right)<\delta$, any side of $P_{I}$ measures less than $\delta$. Thus, the volume is less than $\delta$. Because are $r_{1}+\cdots+r_{d}$ points $b_{i, j}$ and therefore $r_{1}+\cdots+r_{d}$ hyperplanes, we 
obtain

$$
\sum_{P_{I} \in \mathcal{P}_{2}} \mu\left(P_{I}\right)<\delta\left(r_{1}+\cdots+r_{d}\right)
$$

Taking $\delta=\frac{\varepsilon}{4 M\left(r_{1}+\cdots+r_{d}\right)}$, we have

$$
\sum_{I \in\left[t_{1}\right] \times \cdots \times\left[t_{d}\right]} \omega\left(f, P_{I}\right) \mu\left(P_{I}\right) \leq \frac{\varepsilon}{2}+2 M \sum_{P_{I} \in \mathcal{P}_{2}} \mu\left(P_{I}\right)<\frac{\varepsilon}{2}+\frac{\varepsilon}{2}=\varepsilon .
$$

Now we are able to prove Lemma 2.5.

Proof of Lemma 2.5. For every $m \in \mathbb{N}$, let $P^{m}=\left(P_{1}^{m}, \ldots, P_{2 n}^{m}\right)$ be a partition of $[0,1]^{2 n}$, where $P_{i}^{m}=\{0,1 / m, \ldots,(m-1) / m, 1\}$ for every $1 \leq i \leq 2 n$. Thus, every rectangle in $P^{m}$ is a hypercube of sides $1 / \mathrm{m}$ with diameter $\sqrt{3} / \mathrm{m}$. Theorem 2.8 and Corollary 2.7 yields that

$$
\int_{[0,1]^{2 n}} \mathbb{1}_{\mathcal{B}} \mathrm{d} \mu=\lim _{m \rightarrow \infty} \sum_{I \in[m]^{2 n}} \mathbb{1}_{\mathcal{B}}\left(a_{I}\right) \mu\left(P_{I}^{m}\right)
$$

for any choice of $a_{I}$ inside the rectangle $P_{I}^{m}$.

On the other hand, for every integer $m$ let $G_{m}$ be the set of centers of the $m \times m$ grid of size $1 / m$, i.e.,

$$
G_{m}=\left\{\left(\frac{2 i-1}{2 m}, \frac{2 j-1}{2 m}\right): 1 \leq i, j \leq m\right\}
$$

The probability $\mathbb{P}\left(V_{m} \cong B\right)$ is equal to the probability of choosing uniformly and independently $n$ points of the set $G_{m}$. Write $V_{m}=\left\{x_{1}, \ldots, x_{n}\right\}$ with $x_{i} \in G_{m}$, and let $x=$ $\left(x_{1}, \ldots, x_{n}\right) \in[m]^{2 n}$. It turns out that $x$ is the center of some hypercube in $P$. Thus,

$$
\mathbb{P}\left(V_{m} \cong B\right)=\sum_{I \in[m]^{2 n}} \mathbb{1}_{\mathcal{B}}\left(c_{I}\right) \mu\left(P_{I}^{m}\right)
$$

where $c_{I}$ is the center of the hypercube $P_{I}^{m}$. Then by the previous paragraph,

$$
\mathbb{P}(U \cong B)=\int_{[0,1]^{2 n}} \mathbb{1}_{\mathcal{B}} \mathrm{d} \mu=\lim _{m \rightarrow \infty} \sum_{I \in[m]^{2 n}} \mathbb{1}_{\mathcal{B}}\left(c_{I}\right) \mu\left(P_{I}^{m}\right)=\lim _{m \rightarrow \infty} \mathbb{P}\left(V_{m} \cong B\right) .
$$

\subsubsection{Geometric lemma}

Another consequence of $\Gamma[B]$ being open, for $B$ in general position, is that for every configuration $A \cong B$ there exists a small neighbourhood in each point of $A$ such that any transversal is also in $\Gamma[B]$, i.e, isomorphic to $B$. This leads to the following natural definition. 
Definition 2.9. A configuration $X$ is an $\varepsilon$-perturbation of $Y$, if there exists a bijection $\iota$ : $X \rightarrow Y$ such that $\|x-\iota(x)\|<\varepsilon$ for every $x \in X$. For ordered $n$-tuples $U, V$ we say that $U$ is an $\varepsilon$-perturbation of $V$ if $\left\|u_{i}-v_{i}\right\|<\varepsilon$ for every $1 \leq i \leq n$.

One can use Definition 2.9 to restate the definition of $\Gamma[B]$ being open. Indeed, $\Gamma[B]$ is open if for every $A \in \Gamma[B]$, there exists $\varepsilon:=\varepsilon(A)>0$ such that every $\varepsilon$-perturbation of $A$ is in $\Gamma[B]$. That is, every $\varepsilon$-perturbation of $A$ is isomorphic to $A$

Our main lemma in this subsection gives an estimate on the size of $\varepsilon$. For a configuration $X$ define $\Delta_{\min }(X)$ as the minimum area of a triangle with vertices in $X$. This value will be positive because $B$ is in general position.

Lemma 2.10. Let $X \subset[0, L]^{2}$ be a configutation in general position. Then every $\left(\frac{\Delta_{\min }(X)}{L \sqrt{2}}\right)$ perturbation of $X$ is isomorphic to $X$.

The proof relies on the following geometric fact.

Proposition 2.11. Let $A B C$ be a triangle and $l$ a straight line. Define $d(P, l)$ as the minimum distance from a point $P$ to $l$. If $h$ is the minimal height of $A B C$, then

$$
\max \{d(A, l), d(B, l), d(C, l)\} \geq \frac{h}{2} .
$$

Proof. Fix a direction and consider all lines in this direction. Out of all these lines, there are two of them $l_{1}, l_{2}$, each one touching a vertex of $A B C$, such that the gap between them contains $A B C$. It is not hard to see that the optimal line, in the fixed direction, which minimizes the desired function is the line equally spaced to $l_{1}$ and $l_{2}$ (see Figure 2.2).

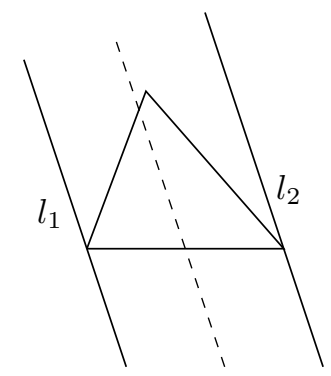

Figure 2.2: The optimal line in a fixed direction (dashed)

Then it follows that for any line $l$ in the fixed direction, $\max \{d(A, l), d(B, l), d(C, l)\} \geq$ $d\left(l_{1}, l_{2}\right) / 2$, where $d\left(l_{1}, l_{2}\right)$ is the distance between lines $l_{1}$ and $l_{2}$. It remains now to prove that $d\left(l_{1}, l_{2}\right) \geq h$. Suppose without loss of generality that $l_{1}$ and $l_{2}$ touch vertices $A$ and $B$, respectively, and that $C$ is on the half upper plane defined by the line passing through $A$ and $B$. Moreover, suppose that $B$ and $C$ are not in the same half open plane determined by the perpendicular line $s$ to $l_{1}, l_{2}$ passing through $A$. We can divide into two cases:

Case 1: $B$ is on $s$.

If $B$ is on $s$, it follows that $d\left(l_{1}, l_{2}\right)=l(A B)$. Since the minimum height is less than or equal the length of any side of $A B C$ we are done. 
Case 2: $B$ is on the lower half open plane determined by $s$.

Let $P$ be the intersection of $s$ with $l_{2}$ and $D$ the foot of the altitude from vertex $A$ (Figure 2.3). It is evident that $\angle A B D \leq \angle A B P$. Using this and that $A B D$ and $A B P$ share the same hypotenuse, we obtain $d\left(l_{1}, l_{2}\right)=A P \geq A D \geq h$.

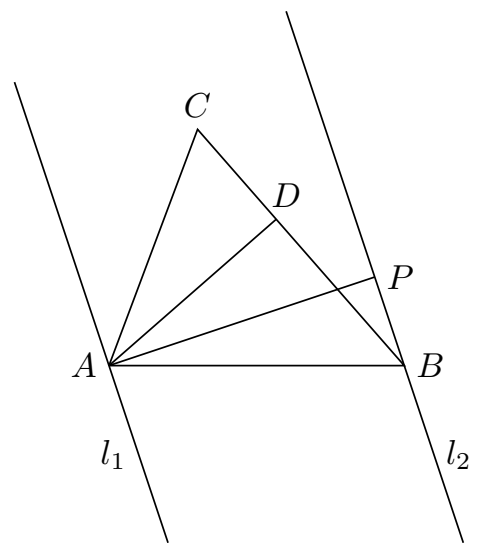

Figure 2.3: $B$ is on the lower half plane of $s$

Proposition 2.11 allows us to show that any sufficient small perturbation of a triangle maintains the signed area. This is sufficient to prove Lemma 2.10 .

Proof of Lemma 2.10. Let $\varepsilon=\frac{\Delta_{\min }(X)}{L \sqrt{2}}$ and write $X=\left\{x_{1}, \ldots, x_{n}\right\}$. Let $Y=\left\{y_{1}, \ldots, y_{n}\right\}$ be an $\varepsilon$-perturbation of $X$, i.e., a configuration of points such that $\left\|x_{i}-y_{i}\right\|<\varepsilon$ for all $1 \leq i \leq n$. Our aim is to show that $\operatorname{sgn}\left[x_{i} x_{j} x_{k}\right]=\operatorname{sgn}\left[y_{i} y_{j} y_{k}\right]$ for every $1 \leq i, j, k \leq n$, therefore $X \cong Y$. Since $X$ is in general position, this is exactly the same as proving that $\left[x_{i} x_{j} x_{k}\right]\left[y_{i} y_{j} y_{k}\right]>0$ for every $1 \leq i, j, k \leq n$. Suppose that it is not true. Then there exist $1 \leq i, j, k \leq n$ such that $\left[x_{i} x_{j} x_{k}\right]\left[y_{i} y_{j} y_{k}\right] \leq 0$. We may assume without loss of generality that $\left[x_{1} x_{2} x_{3}\right]\left[y_{1} y_{2} y_{3}\right] \leq 0$.

For $t \in[0,1]$ and $1 \leq i \leq 3$, let $z_{i}(t)=(1-t) x_{i}+t y_{i}$. This continuous curve represents the straight line trajectory from point $x_{i}=z_{i}(0)$ to point $y_{i}=z_{i}(1)$. Consider the continuous function $\phi:[0,1] \rightarrow \mathbb{R}$ given by

$$
\phi(t)=\left[x_{1} x_{2} x_{3}\right]\left[z_{1}(t) z_{2}(t) z_{3}(t)\right] .
$$

Since $\left[x_{1} x_{2} x_{3}\right] \neq 0$, we have $\phi(0)=\left[x_{1} x_{2} x_{3}\right]^{2}>0$ and $\phi(1)=\left[x_{1} x_{2} x_{3}\right]\left[y_{1} y_{2} y_{3}\right] \leq 0$. Thus, by continuity of $\phi$, there exists $t_{0} \in(0,1]$ such that $\phi\left(t_{0}\right)=0$.

This means that $\left[z_{1}\left(t_{0}\right) z_{2}\left(t_{0}\right) z_{3}\left(t_{0}\right)\right]=0$, or in other words, that $z_{1}\left(t_{0}\right), z_{2}\left(t_{0}\right)$ and $z_{3}\left(t_{0}\right)$ are collinear. Let $l$ be the line passing through these three points. An easy calculation shows that

$$
d\left(x_{i}, l\right) \leq\left\|x_{i}-z_{i}\left(t_{0}\right)\right\| \leq\left\|x_{i}-y_{i}\right\|<\varepsilon, \quad 1 \leq i \leq 3 .
$$


By Proposition 2.11 we have $h / 2 \leq \min \left\{d\left(x_{1}, l\right), d\left(x_{2}, l\right), d\left(x_{3}, l\right)\right\}<\varepsilon$, where $h$ is the minimum height of $x_{1} x_{2} x_{3}$. Thus,

$$
\Delta_{\min }(X) \leq\left|\left[x_{1} x_{2} x_{3}\right]\right|=\frac{h \cdot \max _{1 \leq i, j \leq 3}\left\|x_{i}-x_{j}\right\|}{2} \leq \frac{L h \sqrt{2}}{2},
$$

Hence, we obtain $\varepsilon>\frac{\Delta_{\min }(X)}{L \sqrt{2}}$, which is a contradiction.

\subsection{Transformations preserving order types}

In the last section we discussed a little about the space of realizations of an order type in general position being open (Proposition 2.4). This led to the observation that any small perturbation of a configuration does not change its order type and we were able to estimate how much we can perturb (Lemma 2.5). However, these perturbations are only local and do not give us information when the configurations are far from each other. Thus it is necessary to find other ways to preserve an order type. In this section we will study two families of transformations that have this property.

\subsubsection{Affine transformation}

An affine transformation $T: \mathbb{R}^{2} \rightarrow \mathbb{R}^{2}$ is given by

$$
T(x)=A x+b, \quad \forall x \in \mathbb{R}^{2},
$$

where $A$ is a non-singular $2 \times 2$ real matrix and $b \in \mathbb{R}^{2}$. Types of affine transformations includes homothety, stretching and translation.

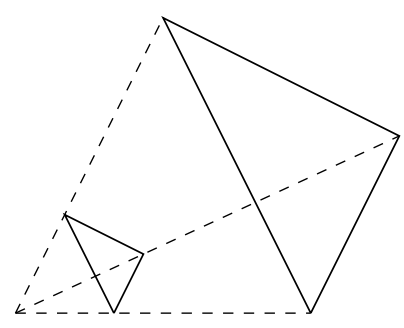

Figure 2.4: A homothety

Affine transformations preserve ratio between area of triangles. One can see that by noticing that

$$
[T(x) T(y) T(z)]=\operatorname{det}(A) \cdot[x y z], \quad \forall x, y, z \in \mathbb{R}^{2} .
$$


Indeed, writing that

$$
A=\left[\begin{array}{ll}
a & b \\
c & d
\end{array}\right], \quad a, b, c, d \in \mathbb{R}
$$

we have

$$
\begin{aligned}
{[T(x) T(y) T(z)] } & =\frac{1}{2}\left|\begin{array}{lll}
(T(x))_{1} & (T(x))_{2} & 1 \\
(T(y))_{1} & (T(y))_{2} & 1 \\
(T(z))_{1} & (T(z))_{2} & 1
\end{array}\right|=\frac{1}{2}\left|\begin{array}{lll}
a x_{1}+b x_{2} & c x_{1}+d x_{2} & 1 \\
a y_{1}+b y_{2} & c y_{1}+d y_{2} & 1 \\
a z_{1}+b z_{2} & c z_{1}+d z_{2} & 1
\end{array}\right| \\
& =\frac{1}{2}\left|\begin{array}{lll}
x_{1} & x_{2} & 1 \\
y_{1} & y_{2} & 1 \\
z_{1} & z_{2} & 1
\end{array}\right|\left|\begin{array}{lll}
a & c & 0 \\
b & d & 0 \\
0 & 0 & 1
\end{array}\right|=\operatorname{det}(A)[x y z] .
\end{aligned}
$$

Therefore, if $x, y, z, u, v, w \in \mathbb{R}^{2}$, then

$$
\frac{[T(x) T(y) T(z)]}{[T(u) T(v) T(w)]}=\frac{\operatorname{det}(A)[x y z]}{\operatorname{det}(A)[u v w]}=\frac{[x y z]}{[u v w]} .
$$

We say that an affine transformation $T$ is positive if $\operatorname{det}(A)>0$. Otherwise, we say that $T$ is negative. Another important observation is that positive affine transformations preserve order types.

Proposition 2.12. If $T: \mathbb{R}^{2} \rightarrow \mathbb{R}^{2}$ is a positive affine transformation, then for every configuration $X$ we have that $T(X) \cong X$.

Proof. Let $X=\left\{x_{1}, \ldots, x_{n}\right\} \subset \mathbb{R}^{2}$ be a configuration of size $n$ and write $T(x)=A x+b$ for $A$ non-singular and $b \in \mathbb{R}^{2}$. We want to prove that $T(X) \cong X$ or that

$$
\chi\left(T\left(x_{i}\right), T\left(x_{j}\right), T\left(x_{k}\right)\right)=\chi\left(x_{i}, x_{j}, x_{k}\right), \quad \forall 1 \leq i, j, k \leq n .
$$

However, this is true since

$$
\chi\left(T\left(x_{i}\right), T\left(x_{j}\right), T\left(x_{k}\right)\right)=\operatorname{sgn}\left[T\left(x_{i}\right) T\left(x_{j}\right) T\left(x_{k}\right)\right]=\operatorname{sgn}\left(\operatorname{det}(A)\left[x_{i} x_{j} x_{k}\right]\right)=\operatorname{sgn}\left[x_{i} x_{j} x_{k}\right],
$$

for $\operatorname{det}(A)>0$.

\subsubsection{Projective transformation}

Given two points $x, y \in \mathbb{R}^{3}$, we say that $x \sim y$ if and only if there exists $\lambda \in \mathbb{R}$ such that $x_{i}=\lambda y_{i}$ for every $1 \leq i \leq 3$. The relation $\sim$ is an equivalence relation and then it makes sense to define the quotient $\mathbb{R}^{3} / \sim$. This quotient is called the real projective plane and is usually denoted by $\mathbb{R} P^{2}$. By this definition, points and lines in the projective plane are the quotient of lines and planes passing through the origin in $\mathbb{R}^{3}$, respectively. Also points and lines are represented in $\mathbb{R} P^{2}$ by triples in $\mathbb{R}^{3}$ and a line $[a, b, c]$ in $\mathbb{R} P^{2}$ passes through a point $[x, y, z] \in \mathbb{R} P^{2}$ if $a x+b y+c z=0$. 
One can view the projective plane as the real plane plus an extra line, which is called the infinity line. In fact, there exists a bijection between the points of the form $[a, b, 1], a, b \in \mathbb{R}$ with the real plane $\mathbb{R}^{2}$. The infinity line consists of all points of the form $[a, b, 0]$ and therefore it is the quotient of the plane $z=0$. One of the major differences created by adding the infinity line is that in projective geometry every two lines intersect in a point and the intersection of parallel lines occurs in the infinity line. For a more detailed and precise introduction we suggest [30] and [32].

A projective transformation $S: \mathbb{R} P^{2} \rightarrow \mathbb{R} P^{2}$ is a transformation given by

$$
S\left(\left[x_{1}, x_{2}, x_{3}\right]\right)=\left[\begin{array}{lll}
a_{11} & a_{12} & a_{13} \\
a_{21} & a_{22} & a_{23} \\
a_{31} & a_{32} & a_{33}
\end{array}\right]\left[\begin{array}{l}
x_{1} \\
x_{2} \\
x_{3}
\end{array}\right]
$$

where $A=\left(a_{i j}\right)$ is a non-singular matrix. That is, a projective transformation is the quotient of a linear transformation in $\mathbb{R}^{3}$.

Projective transformations preserve incidence. They send concurrent lines to concurrent lines, collinear points to collinear points and intersections to their corresponding intersection. However, they do not preserve ratio between lengths and areas. A visual example of such transformations can be found in Figure 2.5. In Figure 2.5 we have a projective transformation that sends plane $P$ to plane $Q$. This transformation also sends the square in $P$ to a convex quadrilateral in $Q$.

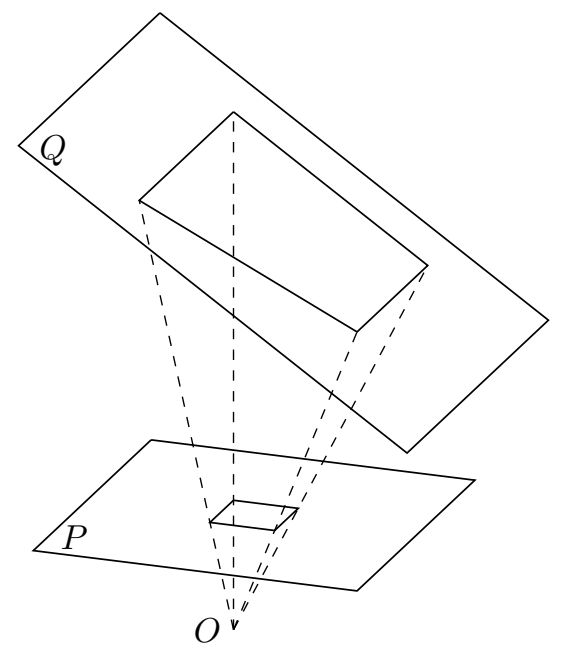

Figure 2.5: A projective transformation

The immersion $i: \mathbb{R}^{2} \hookrightarrow \mathbb{R} P^{2}$ of the real plane in the projective plane given by

$$
i(x, y)=[x, y, 1], \quad \forall x, y \in \mathbb{R}
$$

allows us to consider the real plane as a subset of the projective plane. With this in mind, we consider affine transformations as a particular case of projective transformation. In fact, 
given an affine transformation $T: \mathbb{R}^{2} \rightarrow \mathbb{R}^{2}$,

$$
T(x, y)=\left[\begin{array}{ll}
a & c \\
b & d
\end{array}\right]\left[\begin{array}{l}
x \\
y
\end{array}\right]+\left[\begin{array}{l}
e \\
f
\end{array}\right]=(a x+c y+e, b x+d y+f), \quad \forall x, y \in \mathbb{R}
$$

where the $2 \times 2$ matrix is non-singular and $a, b, c, d, e, f \in \mathbb{R}$. Then the projective transformation $S: \mathbb{R} P^{2} \rightarrow \mathbb{R} P^{2}$ given by

$$
S\left(\left[x_{1}, x_{2}, x_{3}\right]\right)=\left[\begin{array}{lll}
a & c & e \\
b & d & f \\
0 & 0 & 1
\end{array}\right]\left[\begin{array}{l}
x_{1} \\
x_{2} \\
x_{3}
\end{array}\right]=\left[a x_{1}+c x_{2}+e x_{3}, b x_{1}+d x_{2}+f x_{3}, x_{3}\right]
$$

sends points of the form $[x, y, 1]$ to $[a x+c y+e, b x+d y+f, 1]$ and points of the form $[x, y, 0]$ to $[a x+c y, b x+d y, 0]$. That is, restricet to the real plane is the affine transformation $T$ and sends the infinity line to itself.

We will be interested in projective transformations that send configurations in the real plane to configurations also in the real plane. Since our definition of order type deals with arrangement of points and lines and projective transformations preserve incidence, it is natural to consider them as good candidates to preserve order types. Unfortunately, this is not always true. The next proposition shows that every four points general position in $\mathbb{R}^{2} \subset \mathbb{R} P^{2}$ can be sent to the unit square in $\mathbb{R}^{2} \subset \mathbb{R} P^{2}$. The unit square is the square in $\mathbb{R} P^{2}$ whose vertices are $[0,0,1],[1,0,1],[1,1,1],[0,1,1]$.

Proposition 2.13. For every four points in general position in the real plane, there exists a projective transformation that sends them to the unit square.

Proof. Le $A, B, C, D \in \mathbb{R}^{2}$ be four points in general position and let $P, Q$ be the intersection of $A B$ with $C D$ and $A D$ with $B C$, respectively. Consider a projective transformation $S_{1}$ that sends the line $P Q$ to the infinity line. This is always possible, since the line $P Q$ and the infinity line are subspaces of rank 2 in $\mathbb{R}^{3}$.

Note that the configuration $A^{\prime}=S_{1}(A), B^{\prime}=S_{1}(B), C^{\prime}=S_{1}(C), D^{\prime}=S_{1}(D)$ is in the real plane, because $A, B, C, D$ do not belong to the line $P Q$. Let $P^{\prime}=S_{1}(P)$ and $Q^{\prime}=S(Q)$ be the corresponding points to $P$ and $Q$ in the infnity line. Since $S_{1}$ preserves incidence, we obtain that $A^{\prime} B^{\prime}$ and $C^{\prime} D^{\prime}$ intersect on the infinity line. Therefore, $A^{\prime} B^{\prime} \| C^{\prime} D^{\prime}$. Similarly, we obtain $A^{\prime} D^{\prime} \| B^{\prime} C^{\prime}$ and $A^{\prime} B^{\prime} C^{\prime} D^{\prime}$ is a parallelogram. Consider now the affine trasnformation $T$ that sends $B^{\prime}-A^{\prime}$ to $(1,0), D^{\prime}-A^{\prime}$ to $(0,1)$ and $A^{\prime}$ to $(0,0)$. Then, $T$ sends $A^{\prime} B^{\prime} C^{\prime} D^{\prime}$ to the unit square (Here we consider $A^{\prime}, B^{\prime}, C^{\prime}, D^{\prime}$ as points in $\mathbb{R}^{2}$ ). Let $S_{2}$ be the projective transformation that when restricting to the real plane is the affine transformation $T$. Thus $S_{1} \circ S_{2}$ is a projective transformation that sends $A B C D$ into the unit square.

As a consequence, Proposition 2.13 implies that for every convex quadrilateral and triangle with an interior point, there exists a projective transformation sending one to another.

Although the last result shows that not every projective transformation preserves order types, we could still hope that the converse is true, that for every pair of isomorphic con- 
figurations there exists a projective transformation sending one to another. However, this is also not the case. One can notice that the space of configurations obtained by projective transformations of a fixed configuration $X$ is connected, but there exists order types with space of realization disonnected. For instance, in [35] N. White provides a configuration $X$ of size 42 such that $\Gamma[X]$ is disconnected.

Despite this fact, we shall construct a family of order types such that for every pair of configurations, there exists a projective transformation sending one to another. This construction will be helpful later to obtain a lower bound for the Chazelle's encoding problem (see Lemma 2.21).

Definition 2.14. A order type $X=\left\{x_{1}, x_{2}, \ldots, x_{n}\right\}$ with $n \geq 4$ is said to be constructible if $\left\{x_{1}, x_{2}, x_{3}, x_{4}\right\}$ are in general position and for $4<i \leq n, x_{i}$ is the intersection point of exactly two previous lines $x_{p} x_{q}$ and $x_{r} x_{s}$, for $1 \leq p, q, r, s<i$.

For instance, by Definition 2.14 every configuration with 4 poinst in general position is constructible and the configuration in Figure 2.6 is also constructible. However, a convex pentagon is not constructible. In general, for $n>4$ every constructible configuration with $n$ points is not in general position.

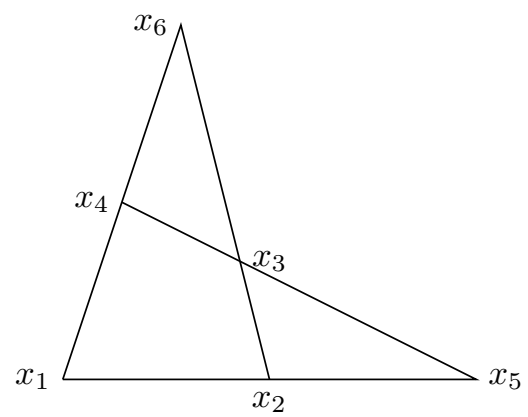

Figure 2.6: A constructible configuration

As we said previously, constructible order types are a family of examples of order types such that for every two isomorphic configuration there exists an projective transformation connecting them.

Proposition 2.15. Let $X$ and $Y$ be isomorphic constructible configuration. Then there exists a projective transformation $S$ such that $Y=S(X)$.

Proof. Let $X=\left\{x_{1}, \ldots, x_{n}\right\}, Y=\left\{y_{1}, \ldots, y_{n}\right\}$ and suppose that

$$
\chi\left(x_{i}, x_{j}, x_{k}\right)=\chi\left(y_{i}, y_{j}, y_{k}\right), \quad \forall 1 \leq i, j, k \leq n .
$$

Since $X$ and $Y$ are constructible, they are determined only by the first four points $\left\{x_{1}, \ldots, x_{4}\right\}$ and $\left\{y_{1}, \ldots, y_{4}\right\}$. Thus we just need to prove that there exists a projective transformation sending $\left\{x_{1}, \ldots, x_{4}\right\}$ to $\left\{y_{1}, \ldots, y_{4}\right\}$. Because these two sets of points are in general position, then by Proposition 2.13 there exists projective transformations $S_{X}, S_{Y}$ send- 
ing $\left\{x_{1}, \ldots, x_{4}\right\},\left\{y_{1}, \ldots, y_{4}\right\}$ to the unit square, respectively. Thus the projective transformation $S=S_{Y}^{-1} \circ S_{X}$ sends $X$ to $Y$.

We already stated that projective transformations does not preserve the ratio between areas of triangles or lenghts of sides. However, they preserve the cross ratio between these measures. Given three points $x, y, z \in \mathbb{R}^{3}$ we can extend the concept of signed area of $x y z$ by

$$
[x y z]=\frac{1}{2}\left[\begin{array}{lll}
x_{1} & x_{2} & x_{3} \\
y_{1} & y_{2} & y_{3} \\
z_{1} & z_{2} & z_{3}
\end{array}\right]
$$

Definition 2.16. Given five points in the projective plane $[x],[y],[z],[u],[v] \in \mathbb{R} P^{2}$ we define the cross-ratio area of these points by

$$
\mathrm{CR}([x] ;[y],[z],[u],[v])=\frac{[x y z][x u v]}{[x y u][x z v]}
$$

where $x, y, z, u, v$ are representatives in $\mathbb{R}^{3}$.

Note that $\mathrm{CR}$ is well defined on $\mathbb{R} P^{2}$. In fact, suppose that we choose other representatives $x^{\prime} \sim x, y^{\prime} \sim y, z^{\prime} \sim z, u^{\prime} \sim u, v^{\prime} \sim v$. Thus, there exists $\lambda_{x}, \lambda_{y}, \lambda_{z}, \lambda_{u}, \lambda_{v} \in \mathbb{R}$ such that $x^{\prime}=\lambda_{x} x, y^{\prime}=\lambda_{y} y, z^{\prime}=\lambda_{z} z, u^{\prime}=\lambda_{u} u, v^{\prime}=\lambda_{v} v$ and then it follows that

$$
\begin{aligned}
\mathrm{CR}\left(\left[x^{\prime}\right] ;\left[y^{\prime}\right],\left[z^{\prime}\right],\left[u^{\prime}\right],\left[v^{\prime}\right]\right) & =\frac{\left[x^{\prime} y^{\prime} z^{\prime}\right]\left[x^{\prime} u^{\prime} v^{\prime}\right]}{\left[x^{\prime} y^{\prime} u^{\prime}\right]\left[x^{\prime} z^{\prime} v^{\prime}\right]}=\frac{\left(\lambda_{x} \lambda_{y} \lambda_{z}[x y z]\right)\left(\lambda_{x} \lambda_{u} \lambda_{v}[x u v]\right)}{\left(\lambda_{x} \lambda_{y} \lambda_{u}[x y u]\right)\left(\lambda_{x} \lambda_{z} \lambda_{v}[x z v]\right)} \\
& =\frac{[x y z][x u v]}{[x y u][x z v]}=\operatorname{CR}([x] ;[y],[z],[u],[v]) .
\end{aligned}
$$

If the points are in the real plane, we can always choose a representative of the form $[a, b, 1]$ with $a, b \in \mathbb{R}$, and in this case the cross-ratio area is exactly the cross ratio area between triangles $x y z, x u v, x y u, x z v$.

Definition 2.16 is similar to the definition of cross-ratio for four points in a line. Let $A, B, C, D$ be four points in a line, then we can define their cross-ratio as

$$
[A, B, C, D]=\frac{A B \cdot C D}{A C \cdot B D}
$$

The next proposition shows that cross-ratio area is preserved by projective transformations.

Proposition 2.17. Let $S$ be a projective transformation and $[x],[y],[z],[u],[v] \in \mathbb{R} P^{2}$ points in the projective plane. Then it follows that

$$
\mathrm{CR}([x] ;[y],[z],[u],[v])=\mathrm{CR}(S([x]) ; S([y]), S([z]), S([u]), S([v]))
$$

Proof. Let $A$ be the non-singular $3 \times 3$ matrix that defines the projective transformation $S$, i.e., $A$ is the matrix such that $S([x])=[A x]$ for every $x \in \mathbb{R}^{3}$. Then by a simple calculation 
we obtain

$$
\begin{aligned}
\mathrm{CR}(S([x]) ; S([y]), S([z]), S([u]), S([v])) & =\mathrm{CR}([A x] ;[A y],[A z],[A u],[A v]) \\
& =\frac{[A x A y A z][A x A u A v]}{[A x A y A u][A x A z A v]}=\frac{\operatorname{det}(A)^{2}[x y z][x u v]}{\operatorname{det}(A)^{2}[x y u][x z v]} \\
& =\frac{[x y z][x u v]}{[x y u][x z v]}=\mathrm{CR}([x] ;[y],[z],[u],[v]) .
\end{aligned}
$$

For a configuration $X$ in the real plane, let

$$
\mathrm{CR}(X)=\max _{x_{i}, x_{j}, x_{k}, x_{l}, x_{m} \in X} \mathrm{CR}\left(x_{i} ; x_{j}, x_{k}, x_{l}, x_{m}\right),
$$

be the cross-ratio of $X$. For an order type $X$ we let

$$
\overline{\mathrm{CR}}(X)=\inf _{Y \cong X} \mathrm{CR}(Y),
$$

be its cross-ratio. One immediate consequence of Proposition 2.17 is that if a projective transformation preserves the order type of a configuration, then it also preserves its crossratio.

\subsection{Chazelle's encoding}

A consequence of Theorem 2.3 is that we can store all order types of size $n$ in $O(n \log n)$ bits. However, it seems difficult to store this information in $O(n \log n)$ bits without losing the geometric and combinatorial properties of order types. Interested in such a problem, B. Chazelle proposed the question of finding the minimum size of a grid that contains all order types in general position of size $n$. Let

$$
\operatorname{gr}(n)=\max \{\operatorname{gr}(B): B \text { order type of size } n\}
$$

be the grid size parameter for the integer $n$.

Note that we shall only consider order types $B$ in general position since there exists order types not in general position with no rational realization. That is, there exists an order type with no configurations with only rational coordinates (see [19], p.33). The problem of determining $\operatorname{gr}(n)$ is known as the Chazelle's encoding problem and it was solved by Goodman, Pollack and Sturmfels [13]. They showed that $\operatorname{gr}(n)$ is double exponential, which is quite unexpected.

Theorem 2.18 ([13]). There exist positive real numbers $c_{1}$ and $c_{2}$ such that for every sufficiently large $n$ the following holds,

$$
2^{2^{c_{1} n}} \leq \operatorname{gr}(n) \leq 2^{2^{c_{2} n}}
$$


In this section, we show their proof of Theorem 2.18. The proof is divided into two parts (Lemmas 2.23 and 2.25). In the first part, we exhibit an order type with gr parameter double exponential in $n$. In the second part, we use a result of algebraic geometry to provide an upper bound.

\subsubsection{The lower bound}

We start by giving an overview of the proof. First we construct a constructible (Definition 2.14) order type $C$ such that its cross-ratio is double exponential in the size of $C$, this is the content of Lemma 2.19. However, the order type $C$ will not be in general position. In order to solve that, we use a method developed by Sturmfels and White [20] to create an order type $B$ in general position called scattering (Lemma 2.21). By Proposition 2.22 we obtain that the cross-ratio of $B$ is as big as the cross-ratio of $C$. Finally, we provide a relation between cross-ratios and the grid parameter, which will imply our lower bound. Now we give more details.

Lemma 2.19. For every integer $r$, there exists a constructible order type $C$ of size $3 r+8$ with $\overline{\mathrm{CR}}(C) \geq 2^{2^{r}}$.

Proof. Consider the configuration $X_{4}=\left\{x_{1}, x_{2}, x_{3}, x_{4}\right\}$ in the projective plane, where $x_{1}=$ $[1,0,0], x_{2}=[0,1,0], x_{3}=[1,0,1]$ and $x_{4}=[0,1,1]$. The configuration $X_{4}$ is in general position since no three points are collinear. We construct $X_{8}$ by adding 4 new points in $X_{4}$. These new points are defined as follows

$$
\begin{aligned}
& x_{5}=[0,0,1]=[1,0,0][1,0,1] \cap[0,1,0][0,1,1], \\
& x_{6}=[1,1,1]=[1,0,0][0,1,1] \cap[0,1,0][1,0,1], \\
& x_{7}=[1,-1,0]=[1,0,1][1,1,0] \cap[1,0,0][0,1,0], \\
& x_{8}=[2,0,1]=[1,1,1][1,-1,0] \cap[1,0,1][1,0,0] .
\end{aligned}
$$

Now note that from $[1,0,0],[0,1,0],[1,0,1],[0,1,1],[1,-1,0]$ and $[a, 0,1]$ we can construct the point $\left[a^{2}, 0,1\right]$. This is done by constructing

$$
\begin{aligned}
{[0, a, 1] } & =[a, 0,1][1,-1,0] \cap[0,1,0][0,1,1], \\
{[a,-1,0] } & =[a, 0,1][0,1,1] \cap[1,0,0][0,1,0], \\
{\left[a^{2}, 0,1\right] } & =[0, a, 1][a,-1,0] \cap[1,0,0][1,0,1],
\end{aligned}
$$

as shown in Figure 2.7.

Thus we can define recursively $3 r+8$ points in the following way. For every $1 \leq t \leq r$, we have

$$
\begin{aligned}
& x_{3 t+6}=x_{3(t-1)+8} x_{7} \cap x_{2} x_{4}=x_{3(t-1)+8}[1,-1,0] \cap[0,1,0][0,1,1], \\
& x_{3 t+7}=x_{3(t-1)+8} x_{4} \cap x_{1} x_{2}=x_{3(t-1)+8}[0,1,1] \cap[1,0,0][0,1,0], \\
& x_{3 t+8}=x_{3 t+6} x_{3 t+7} \cap x_{1} x_{3}=x_{3 t+6} x_{3 t+7} \cap[1,0,0][1,0,1] .
\end{aligned}
$$




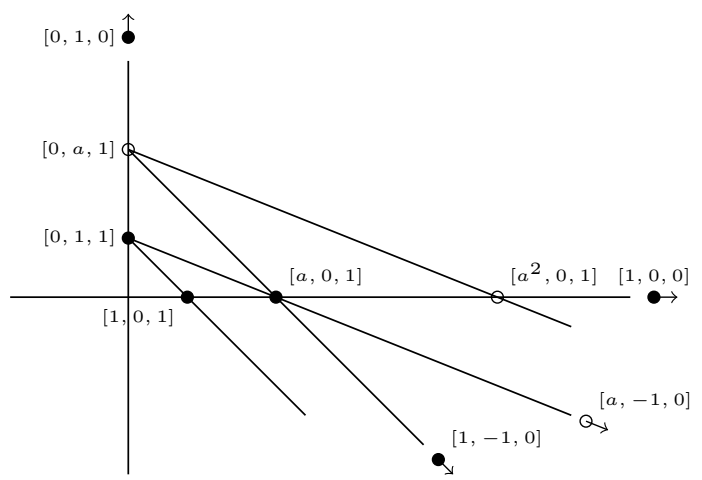

Figure 2.7: Constructing $\left[a^{2}, 0,1\right]$.

Let $X_{3 r+8}$ be the configuration obtained by these $3 r+8$ points. A simple induction using the contruction of Figure 2.7 shows that $X_{3 r+8}$ contains the points $x_{4}=[0,1,1], x_{5}=$ $[0,0,1], x_{3 r+8}=\left[2^{2^{r}}, 0,1\right], x_{3}=[1,0,1]$ and $x_{1}=[1,0,0]$. Then we can estimate the cross-ratio of $X_{3 r+8}$ by

$$
\begin{aligned}
\mathrm{CR}\left(X_{3 r+8}\right) & \geq \operatorname{CR}\left(x_{4} ; x_{5}, x_{3 r+8}, x_{3}, x_{1}\right)=\frac{\left[x_{4} x_{5} x_{3 r+8}\right]\left[x_{4} x_{3} x_{1}\right]}{\left[x_{4} x_{5} x_{3}\right]\left[x_{4} x_{3 r+8} x_{1}\right]} \\
& =\left|\begin{array}{ccc}
0 & 1 & 1 \\
0 & 0 & 1 \\
2^{2^{r}} & 0 & 1
\end{array}\right|\left|\begin{array}{lll}
0 & 1 & 1 \\
1 & 0 & 1 \\
1 & 0 & 0
\end{array}\right| /\left|\begin{array}{ccc}
0 & 1 & 1 \\
0 & 0 & 1 \\
1 & 0 & 1
\end{array}\right|\left|\begin{array}{ccc}
0 & 1 & 1 \\
2^{2^{r}} & 0 & 1 \\
1 & 0 & 0
\end{array}\right|=2^{2^{r}} .
\end{aligned}
$$

Finally, let $s$ be a line in $\mathbb{R} P^{2}$ such that $s$ contains no points of $X_{3 r+8}$. The projective transformation $S: \mathbb{R} P^{2} \rightarrow \mathbb{R} P^{2}$ that sends $s$ to the infinity line, sends $X_{3 r+8}$ to a configuration $C$ in the real plane. We claim that every configuration $A$ isomorphic to $C$ is such that $\operatorname{CR}(A) \geq 2^{2^{r}}$. Indeed, since $X_{3 r+8}$ is determined by its first four points and projective transformations preserve incidence we have that the order type of $C$ is constructible. Thus, by Proposition 2.15, there exists a projective transformation $T: \mathbb{R} P^{2} \rightarrow \mathbb{R} P^{2}$ such that $A=T(C)$. By Proposition 2.17, we obtain

$$
\mathrm{CR}(A)=\mathrm{CR}(T(C))=\mathrm{CR}\left((T \circ S)\left(X_{3 r+8}\right)\right)=\mathrm{CR}\left(X_{3 r+8}\right) \geq 2^{2^{r}}
$$

therefore $\overline{\mathrm{CR}}(C) \geq 2^{2^{r}}$.

The order type $C$ obtained in Lemma 2.19 is not in general position. In order to obtain a order type in general position we will use a method developed by Sturmfels and White [20] called scattering. The method basically consists of replacing every point of a constructible order type by four points such that the original point is contained in the convex hull of the new ones.

Definition 2.20. A scattering of a point $x \in \mathbb{R}^{2}$ is the process of replacing $x$ by four points $x_{1}, x_{2}, x_{3}, x_{4}$ such that $x$ is in the convex hull of $\left\{x_{1}, x_{2}, x_{3}, x_{4}\right\}$. We say that a configuration $Y$ is scattered from $X$ if $Y$ is obtained from $X$ by scattering some of its points. 
We say that an order type $Y$ is scattered from $X$ if for every configuration $A \cong Y$, there exists a configuration $B \cong X$ such that $A$ is scattered from $B$.

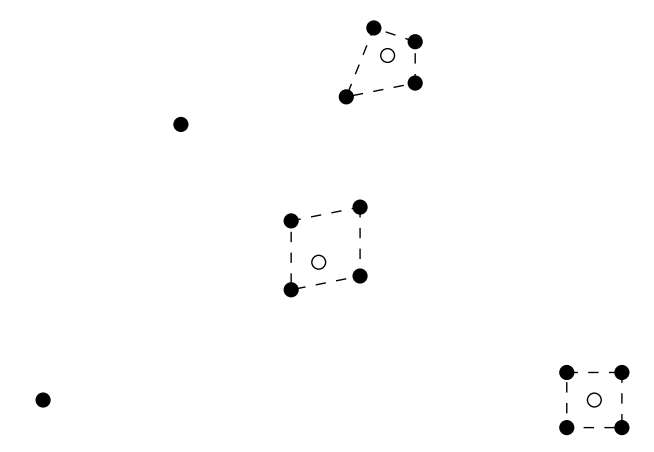

Figure 2.8: Scattering of a configuration

Figure 2.8 shows a scattering of a configuration. The white points are the points of the original configuration that were replaced by scattering. The next lemma shows that for every constructible order type $X$ we can always create an order type $X^{\prime}$ in general position by this process.

Lemma 2.21. Let $X$ be a constructible order type of size $n$. Then there exists an order type $X^{\prime}$ in general position, of size $4 n-12$, scattered from $X$.

Proof. Write $X=\left\{x_{1}, \ldots, x_{n}\right\}$. We will construct a sequence of order types $Y_{n}, Y_{n-1}, \ldots, Y_{4}$ in the following way. Let $Y_{n} \cong X$. For every $4 \leq i \leq n-1$, we construct an order type $Y_{i}$ of size $4 n-3 i$ scattered from $Y_{i+1}$ by replacing $x_{i+1}$ with new points $x_{i+1,1}, x_{i+1,2}, x_{i+1,3}, x_{i+1,4}$ such that $x_{i+1}$ is in the interior of the convex hull of those points. The order type $Y_{i}$ also has the property that any collinear triple of points is in $\left\{x_{1}, \ldots, x_{i}\right\}$.

In order to do that, consider the configuration $Y_{i+1}=\left\{x_{1}, \ldots, x_{i+1}, x_{i+2,1}, \ldots, \ldots, x_{n, 4}\right\}$. Since $X$ is constructible and $i+1>4$, we have that $x_{i+1}$ is the intersection point of two lines $x_{p} x_{q} \cap x_{r} x_{s}$ for $1 \leq p, q, r, s \leq i$. Let $Y_{i}$ be the configuration obtained by replacing $x_{i+1}$ with

$$
\begin{aligned}
& x_{i+1,1}=x_{i+1}+\varepsilon_{1}\left(x_{p}-x_{q}\right)+\varepsilon_{2}\left(x_{r}-x_{s}\right), \\
& x_{i+1,2}=x_{i+1}-\varepsilon_{3}\left(x_{p}-x_{q}\right)+\varepsilon_{4}\left(x_{r}-x_{s}\right), \\
& x_{i+1,3}=x_{i+1}-\varepsilon_{5}\left(x_{p}-x_{q}\right)-\varepsilon_{6}\left(x_{r}-x_{s}\right), \\
& x_{i+1,4}=x_{i+1}+\varepsilon_{7}\left(x_{p}-x_{q}\right)-\varepsilon_{8}\left(x_{r}-x_{s}\right),
\end{aligned}
$$

where $\varepsilon_{1}, \ldots, \varepsilon_{8}>0$ are small enough such that $\chi\left(x_{i+1, j}, y, z\right)=\chi\left(x_{i+1}, y, z\right)$ for every $1 \leq j \leq 4$ and $\{y, z\} \subset Y_{i+1} \backslash\left\{x_{i+1}\right\}$ such that $\left\{x_{i+1}, y, z\right\}$ are not collinear. Also we choose $\varepsilon_{1}, \ldots, \varepsilon_{8}$ in such a way that every line determined by the set $\left\{x_{i+1,1}, \ldots, x_{i+1,4}\right\}$ does not intersect any point of $Y_{i}$. This can be done, for instance, by choosing $\varepsilon_{1} \gg \varepsilon_{2} \gg$ $\cdots \gg \varepsilon_{8}$. If $\chi\left(x_{i+1}, y, z\right)=0$, then because $\varepsilon_{1}, \ldots, \varepsilon_{8}$ are all greater than zero we have that $\chi\left(x_{i+1, j}, y, z\right) \neq 0$, for $1 \leq j \leq 4$. This implies that every collinear triple in $Y_{i}$ does 
not contain $x_{i+1,1}, \ldots, x_{i+1,4}$ as a element and therefore by induction every collinear triple of $Y_{i}$ is in $\left\{x_{1}, \ldots, x_{i}\right\}$. It remains to prove that the order type $Y_{i}$ is scattered from $Y_{i+1}$, that is, for every configuration $A \cong Y_{i}$, there exists a configuration $B \cong Y_{i+1}$ such that $A$ is scattered from $B$.

We define a function $\phi: \Gamma\left[Y_{i}\right] \rightarrow\left(\mathbb{R}^{2}\right)^{4 n-3 i-3}$ in the following way. Let $A \in \Gamma\left[Y_{i}\right]$ and $a_{p}, a_{q}, a_{r}, a_{s}, a_{i+1,1}, a_{i+1,2}, a_{i+1,3}, a_{i+1,4} \in A$ be the points corresponding to $x_{p}, x_{q}$, $x_{r}, x_{s}, x_{i+1,1}, x_{i+1,2}, x_{i+1,3}, x_{i+1,4} \in Y_{i}$, respectively. Define $\phi(A)$ as the configuration obtained by removing $a_{i+1,1}, a_{i+1,2}, a_{i+1,3}$ and $a_{i+1,4}$ from $A$ and adding the point $a_{i+1}=$ $a_{p} a_{q} \cap a_{r} a_{s}$. First note that $A$ is scattered from $\phi(A)$. Indeed, because $A \cong Y_{i}$ we have that $\left\{a_{i+1,1}, \ldots, a_{i+1,4}\right\}$ is a convex quadrilateral and $a_{p} a_{q}, a_{r} a_{s}$ intersects in its interior. Therefore $\left\{a_{i+1,1}, \ldots, a_{i+1,4}\right\}$ is a scattering of $a_{i+1}$.

Now we claim that $\phi(A) \in \Gamma\left[Y_{i+1}\right]$. Let $\iota: Y_{i} \rightarrow A$ be a bijection such that $\chi(x, y, z)=$ $\chi(\iota(x), \iota(y), \iota(z))$ for every $(x, y, z) \in Y_{i}^{3}$. Then since that $\chi\left(x_{i+1}, y, z\right)=\chi\left(a_{i+1,1}, \iota(y), \iota(z)\right)=$ $\cdots=\chi\left(a_{i+1,4}, \iota(y), \iota(z)\right)$ for every $y, z \in Y_{i+1} \backslash\left\{x_{i+1}\right\}$ such that $\left\{x_{i+1}, y, z \neq 0\right\}$ are not collinear and $a_{i+1}$ is in the interior of $\left\{a_{i+1,1}, \ldots, a_{i+1,4}\right\}$, we obtain that $\chi\left(a_{i+1}, \iota(y), \iota(z)\right)=$ $\chi\left(x_{i+1}, y, z\right)$. If $\chi\left(x_{i+1}, y, z\right)=0$ and $y, z \in Y_{i+1} \backslash\left\{x_{i+1}\right\}$, then $\left\{x_{i+1}, y, z\right\}$ are collinear. By Definition 2.14, this implies that $y, z$ are on the lines $x_{p} x_{q}$ or $x_{s} x_{r}$. Therefore $\iota(y), \iota(z)$ are on the lines $a_{p} a_{q}$ or $a_{s} a_{r}$, which yields $\chi\left(a_{i+1}, \iota(y), \iota(z)\right)=0$. Thus the function $\tilde{\iota}: Y_{i+1} \rightarrow \phi(A)$ defined by $\tilde{\iota}\left(x_{i+1}\right)=a_{i+1}$ and $\tilde{\iota}(y)=\iota(y)$ for $y \in Y_{i+1} \backslash\left\{x_{i+1}\right\}$ is such that

$$
\chi(x, y, z)=\chi(\tilde{\iota}(x), \tilde{\iota}(y), \tilde{\iota}(z)), \quad \forall x, y, z \in Y_{i+1}
$$

and $\phi(A) \cong Y_{i+1}$. This concludes that $Y_{i}$ is scattered from $Y_{i+1}$.

Finally, let $X^{\prime} \cong Y_{4}$. By induction, we obtain that a collinear triple in $X^{\prime}$ only contain points of $\left\{x_{1}, x_{2}, x_{3}, x_{4}\right\}$. However, the subconfiguration $\left\{x_{1}, x_{2}, x_{3}, x_{4}\right\}$ is in general position. Therefore, $X^{\prime}$ is in general position. Also because scattering is transitive, we obtain that $X^{\prime}$ is scattered from $X$ and $X^{\prime}$ has size $n+3(n-4)=4 n-12$.

The next proposition shows that scattering configurations only increases its cross-ratio.

Proposition 2.22. Let $x, y, z, u, v \in \mathbb{R}^{2}$ such that $y, z, u, v$ are collinear. Consider the twenty points $\left\{x_{i}\right\}_{1 \leq i \leq 4},\left\{y_{i}\right\}_{1 \leq i \leq 4},\left\{z_{i}\right\}_{1 \leq i \leq 4},\left\{u_{i}\right\}_{1 \leq i \leq 4},\left\{v_{i}\right\}_{1 \leq i \leq 4}$ obtained by scattering $x, y, z, u, v$. Then there exists $1 \leq i, j, k, l, m \leq 4$ such that

$$
\mathrm{CR}(x ; y, z, u, v) \leq \mathrm{CR}\left(x_{i} ; y_{j}, z_{k}, u_{l}, v_{m}\right)
$$

Proof. First note that because $y, z, u, v$ are collinear, we have that

$$
\mathrm{CR}(x ; y, z, u, v)=\frac{[x y z][x u v]}{[x y u][x z v]}=\frac{|y-z| \cdot|u-v|}{|y-u| \cdot|z-v|}=[y, z, u, v]
$$

where $[y, z, u, v]$ is the cross-ratio between these four points. This is a consequence of the fact that $x y z, x y u, x u v$ and $x z v$ have the same height. Therefore, the cross-ratio does not 
depend of $x$ and we obtain that

$$
\mathrm{CR}(x ; y, z, u, v)=\mathrm{CR}\left(x_{i} ; y, z, u, v\right), \quad \forall 1 \leq i \leq 4 .
$$

Another important observation is that we can compute the cross-ratio only using the angles determined by $x$. Let $\theta_{y z}, \theta_{u v}, \theta_{y u}, \theta_{z v}$ be the angles determined by the lines $x y$ with $x z, x u$ with $x v, x y$ with $x u$ and $x z$ with $x v$, respectively. Thus,

$$
\begin{aligned}
\mathrm{CR}(x ; y, z, u, v) & =\frac{[x y z][x u v]}{[x y u][x z v]}=\frac{\left(|x-y| \cdot|x-z| \sin \theta_{y z}\right)\left(|x-u| \cdot|x-v| \sin \theta_{u v}\right)}{\left(|x-y| \cdot|x-u| \sin \theta_{y u}\right)\left(|x-z| \cdot|x-v| \sin \theta_{z v}\right)} \\
& =\frac{\sin \theta_{y z} \sin \theta_{u v}}{\sin \theta_{y u} \sin \theta_{z v}} .
\end{aligned}
$$

Fix the point $x_{1}$. Suppose without loss of generality that $y, z, u, v$ are in the order shown in Figure 2.9. Since $y$ is in the convex hull determined by $y_{1}, y_{2}, y_{3}, y_{4}$, there exists a choice of $y_{j}$ that increases $\theta_{y z}, \theta_{y u}$ and a choice of $y_{j}$ that decreases $\theta_{y z}, \theta_{y u}$. It turns out, by a quick computation, that one of those choices does not decrease the ratio $\sin \theta_{y z} / \sin \theta_{y u}$. Therefore, for the chosen $j$, we have

$$
\mathrm{CR}\left(x_{1} ; y, z, u, v\right) \leq \mathrm{CR}\left(x_{1} ; y_{j}, z, u, v\right) .
$$

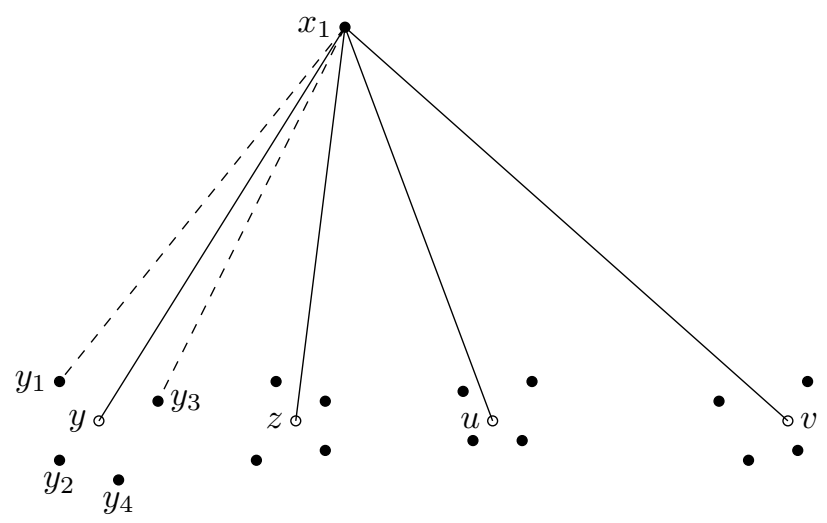

Figure 2.9: Choosing $y_{j}$

Fixing $y_{j}$ and repeating the process for $z, u, v$ we can choose $z_{k}, u_{l}, v_{m}$ such that

$$
\mathrm{CR}(x ; y, z, u, v) \leq \mathrm{CR}\left(x_{1} ; y_{j}, z, u, v\right) \leq \mathrm{CR}\left(x_{1} ; y_{j}, z_{k}, u_{l}, v_{m}\right),
$$

which concludes the proof.

Now we are able to provide a lower bound for $\operatorname{gr}(n)$.

Lemma 2.23. For $n \geq 240$, there exists an order type $B$ of size $n$ such that

$$
\operatorname{gr}(B) \geq 2^{2^{n / 15}}
$$


Proof. The proof now is just a compilation of the previous results. Consider the order type $C$ of $3 r+8$ points obtained by Lemma 2.19. By Lemma 2.21, there exists an order type $B$ in general position, of $12 r+20$ points, such that $B$ is scattered from $C$.

Let $X \cong B$ be a configuration of order type $B$. Since $B$ is scattered from $C$, there exists a configuration $Y \cong C$ such that $X$ is scattered from $Y$. However, as we already saw in the proof of Lemma 2.19, there exists a projective transformation $S: \mathbb{R} P^{2} \rightarrow \mathbb{R} P^{2}$ such that $Y=S\left(X_{3 r+8}\right)$. Let $x=S([0,1,1]), y=S([0,0,1]), z=S\left(\left[2^{2^{r}}, 0,1\right]\right), u=$ $S([1,0,1]), v=S([1,0,0])$ be the corresponding points in $Y$. By Proposition 2.17, we obtain that

$$
\mathrm{CR}(Y) \geq \operatorname{CR}(x ; y, z, u, v)=2^{2^{r}} .
$$

Also $y, z, u, v$ are collinear because projective transformations preserve incidence. Therefore, by Proposition 2.22, there exists points $x_{i}, y_{j}, z_{k}, u_{l}, v_{m} \in X$ such that

$$
\mathrm{CR}(X) \geq \mathrm{CR}\left(x_{i} ; y_{j}, z_{k}, u_{l}, v_{m}\right) \geq \mathrm{CR}(x ; y, z, u, v)=2^{2^{r}} .
$$

Thus, by the generality of $X$, we obtain $\overline{C R}(B) \geq 2^{2^{r}}$.

Now let $M=\operatorname{gr}(B)$ and suppose that $X \cong B$ is a configuration isomorphic to $B$ with vertices in $[M]^{2}$. Then it is possible to estimate $\mathrm{CR}(X)$ by the grid. Indeed,

$$
\mathrm{CR}(X)=\max _{x, y, z, u, v \in X} \frac{[x y z][x u v]}{[x y u][x z v]} \leq \frac{\frac{M^{2}}{2} \cdot \frac{M^{2}}{2}}{\frac{1}{2} \cdot \frac{1}{2}}=M^{4} .
$$

Therefore,

$$
M \geq \mathrm{CR}(X)^{1 / 4} \geq \overline{\mathrm{CR}}(B)^{1 / 4} \geq 2^{2^{r-2}}
$$

and we are done by setting $r=\frac{1}{12}(n-20)$.

\subsubsection{The upper bound}

For the upper bound we will use the following result by Grigor'ev and Vorobjov [18] on solutions of simultaneous inequalities of a semi-algebraic set.

Theorem 2.24 ([18], Lemma 10). Suppose the polynomials $h_{1}, \ldots, h_{k} \in \mathbb{Z}\left[X_{1}, \ldots, X_{n}\right]$ satisfy the bounds deg$\left(h_{i}\right) \leq d$ and all coefficients are smaller in absolute value than $M$. If $\mathcal{W}$ is any connected component of the semi-algebraic set defined by the system $h_{1} \geq$ $0, \ldots, h_{k} \geq 0$, then $\mathcal{W}$ intersects the ball in $\mathbb{R}^{n}$ of radius $R=\exp \left((\log M+\log k)\left(d^{q n}\right)\right)$ centered at the origin for some natural number $q$ that does not depend on $h_{1}, \ldots, h_{k}$.

As a consequence we can prove the following result.

Lemma 2.25. There exists a positive constant $c$ such that for sufficiently large $n$ the fol- 
lowing holds. If $X$ is an order type of size $n$, then it follows that

$$
\operatorname{gr}(X) \leq 2^{2^{c n}}
$$

Proof. Let $X$ be an order type of size $n$. Our goal is to describe the set $\Gamma[X]$ as a semialgebraic set as in Theorem 2.24. However, the space $\Gamma[X]$ is defined as a union of systems composed by strict inequalities. Nevertheless, one can circumvent this issue by considering a smaller subset of $\Gamma[X]$.

Fix an ordering $X=\left(x_{1}, \ldots, x_{n}\right)$ and consider the subset $\mathcal{W}^{\prime}=\left\{A \in \mathbb{R}^{2 n}: \chi_{A}=\chi_{X}\right\} \subset$ $\Gamma[X]$. Note that the set $\mathcal{W}^{\prime}$ is represented by a system of the form

$$
\chi\left(a_{i}, a_{j}, a_{k}\right)=\chi\left(x_{i}, x_{j}, x_{k}\right), \quad \forall a_{i}, a_{j}, a_{k} \in A,
$$

where these functions assume values $\{-,+\}$ when the indices are distinct. In other words, we can represent $\mathcal{W}^{\prime}$ by a system of $\left(\begin{array}{l}n \\ 3\end{array}\right)$ strict inequalities given by

$$
h_{i j k}\left(a_{i}, a_{j}, a_{k}\right)>0, \quad \forall 1 \leq i<j<k \leq n
$$

where $h_{i j k} \in \mathbb{Z}\left[X_{1}, \ldots, X_{6}\right]$ are polynomials such that $\left|h_{i j k}(x, y, z)\right|=|[x y z]|$ for $x, y, z \in \mathbb{R}^{2}$. Therefore, by the definition of $\chi, h_{i j k}$ is a polynomial with 6 variables, maximum absolute value of a coefficient 1 and degree 2 .

Now let $\mathcal{W}$ be the set of solutions of the following system

$$
h_{i j k}\left(a_{i}, a_{j}, a_{k}\right) \geq 1, \quad \forall 1 \leq i<j<k \leq n,
$$

for the same polynomials defined earlier. Note that a solution to this system is a solution to the previous one. That is, $\mathcal{W} \subset \mathcal{W}^{\prime}$. Therefore we can use Theorem 2.24 to obtain a configuration $A \in \mathcal{W} \subset \mathcal{W}^{\prime} \subset \Gamma[X]$ with bounded size. Indeed, by Theorem 2.24, we have that $\mathcal{W}$ intersects a ball centered at the origin of radius

$$
R=e^{(\log M+\log k)\left(d^{2 q n}\right)} \leq e^{3 \cdot 2^{2 q n} \log n} \leq 2^{2^{3 q n}}
$$

for sufficiently large $n$. This implies that there exists a configuration $A \cong X$ inside $[-R, R]^{2}$. By doing a translation, we can actually assume that $A$ is inside the $[0,2 R]^{2}$ square. Also because $A \subset \mathcal{W}$, we have that

$$
\left|\left[a_{i} a_{j} a_{k}\right]\right|=\left|h_{i j k}\left(a_{i}, a_{j}, a_{k}\right)\right| \geq 1,
$$

and therefore $\Delta_{\min }(A) \geq 1$, where $\Delta_{\min }(A)$ is the minimum area of a triangle in $A$.

Consider the configuration obtained by a dilation $A^{\prime}=\{2 R \cdot a: a \in A\}$. Since dilation is an affine transformation, we have that $A^{\prime} \cong A$. Also note that $A \subset\left[0,4 R^{2}\right]$ and $\Delta_{\min }\left(A^{\prime}\right) \geq$ $4 R^{2}$. Therefore, by Lemma 2.10, every $\left(\frac{\sqrt{2}}{2}\right)$-perturbation of $A^{\prime}$ is isomorphic to $X$. Since every point in $\mathbb{R}^{2}$ is at distance less than $\frac{\sqrt{2}}{2}$ from a lattice point, there exists a configuration 
in $\left[\left\lceil 4 R^{2}\right\rceil+1\right]^{2}$ isomorphic to $X$. Thus we conlude that

$$
\operatorname{gr}(X) \leq 4 R^{2}+2 \leq 5 R^{2} \leq 2^{2^{7 q n}} .
$$

for sufficiently large $n$ 


\section{Chapter 3}

\section{Extremal Results}

We study now extremal aspects of order types. One of the main topics in extremal combinatorics is to study how large or small a collection of finite objects satisfying certain property can be. There are many examples of objects and properties that can be studied in this way. For instance, one of the major theorems in the field is the Erdös-Stone theorem [11], which determines the maximal number of edges in a graph $G$ without containing a fixed subgraph $H$. In this work, our objects will be order types in general position and from now on, unless otherwise stated, we assume that all configuration and order types are in general position. We are interested in two different extremal questions.

The first one deals with the minimal size of configuration such that every dense subset contains a copy of a fixed order type.

Definition 3.1. Given two configuration $A, B \in \mathbb{R}^{2}$ and a real number $\alpha>0$, we say that $A \rightarrow_{\alpha} B$ if and only if for every $X \subset A$ with $|X| \geq \alpha|A|$, there exists a subset $Y \subset X$ such that $B \cong Y$. In other words, $A \rightarrow_{\alpha} B$ if and only if every $\alpha$-proportion of $A$ contains a copy of $B$.

Let $\alpha=2 / 3$ and $B$ be the order type of a triangle with an interior point. Then Figure 3.1 is an example of configuration $A$ such that $A \rightarrow_{\alpha} B$. The reader is invited to check that every subset of size 6 of $A$ contains a triangle with an interior point.

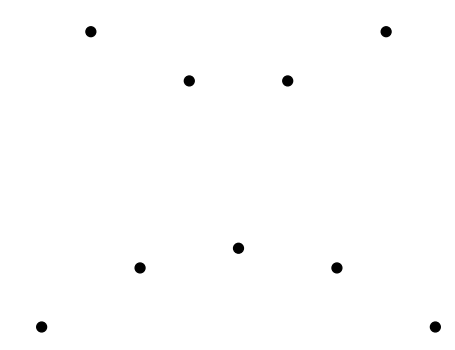

Figure 3.1: Configuration $A$

For an order type $B$ of $n$ points and a real number $\alpha>0$, let

$$
f(B, \alpha)=\min \left\{|A|: A \rightarrow_{\alpha} B\right\}
$$


be the minimum size of an configuration such that every $\alpha$-proportion contains a copy of $B$. Also let

$$
f(n, \alpha)=\max \{f(B, \alpha): B \text { order type with } n \text { points }\}
$$

be the maximum of $f$ over all order types of size $n$. A natural problem is to estimate those two functions.

For instance, if $B$ is a convex $n$-gon, then $f(B, \alpha)=\left\lceil\frac{n}{\alpha}\right\rceil$. In fact, if $A$ is a configuration such that $A \rightarrow_{\alpha} B$, then every $\alpha$-proportion of $A$ contains a copy of $B$. Therefore $\alpha|A| \geq$ $|B|=n$ and we obtain that $f(B, \alpha) \geq\left\lceil\frac{n}{\alpha}\right\rceil$. Now let $C$ be a convex $\left\lceil\frac{n}{\alpha}\right\rceil$-gon. Then every $\alpha$ proportion of $C$ is convex and has size at least $n$. Therefore $C \rightarrow_{\alpha} B$ and $f(B, \alpha) \leq\left\lceil\frac{n}{\alpha}\right\rceil$. In Section 3.1 we shall explore more on this problem, providing general bounds and also studying some specific configurations.

The second problem deals with the maximal subset of the grid without containing a copy of a fixed order type. For an order type $B$ and an integer $N$ let

$$
\operatorname{ex}(N, B)=\max \left\{|A|: B \not \subset A \subset[N]^{2}\right\}
$$

be the maximum size of a subset of $[N]^{2}$ without a copy of $B$. The natural question is to determine bounds for $\operatorname{ex}(N, B)$.

For instance, if $N=3$ and $B$ is a triangle with an interior point, then Figure 3.2 shows a subset with 8 points without a copy of $B$. This proves that $\operatorname{ex}(3, B)=8$.

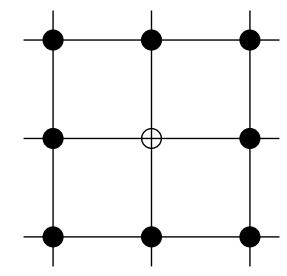

Figure 3.2: $\operatorname{ex}(3, B)=8$

In Section 3.2 we shall give a general bound for the extremal number of a configuration and a sharper bound for the convex case. We also draw a connection between these two extremal problems, showing that ideas on the first one can be helpful to the second one.

\subsection{Density problem in the plane}

Now we give a proof of Theorem 1.2. We divide the proof into two lemmas, which correspond to the upper and lower bound. The proof of the upper bound is based on the observation that order types admit blow-ups, while the lower bound is based on the corresponding graph problem studied by Brown and Rödl [6]. At the end, we provide a family of configurations which shows that the asymptotic behavior of $f$ as a function of $n$ depends on $\alpha$. 


\subsubsection{The upper bound}

In section 2.1 it was shown that the space of realizations of an order type in general position is open (Proposition 2.4). In particular, for every configuration $B$ there exists $\varepsilon$ such that every $\varepsilon$-perturbation of $B$ is isomorphic to it. This remark allows us to show the existence of blow-ups of configurations.

Definition 3.2. A configuration $Y$ is a $k$-blow-up of a configuration $X$ of size $n$ if $Y$ can be partitioned into sets $Y=Y_{1} \cup \cdots \cup Y_{n}$ with $\left|Y_{1}\right|=\cdots=\left|Y_{n}\right|=k$ such that every transversal is isomorphic to $X$, i.e., every set $\left\{y_{1}, \ldots, y_{n}\right\} \subset Y$, with $y_{i} \in Y_{i}$, is isomorphic to $X$.

$A$ configuration $Z$ is a $Y$-blow-up of $X$ if $Z$ can be partitioned into sets $Z=Z_{1} \cup \cdots \cup$ $Z_{n}$ with $Z_{1} \cong \ldots \cong Z_{n} \cong Y$ such that every transversal is isomorphic to $X$, i.e., every set $\left\{z_{1}, \ldots, z_{n}\right\} \subset Z$, with $z_{i} \in Z_{i}$, is isomorphic to $X$.

Given a configuration $X$, there is an easy way to construct blow-ups from it. Let $\varepsilon$ be a positive real number such that every $\varepsilon$-perturbation of $X$ is isomorphic to $X$ and choose $Z_{k}$ as any $k$-subset of $B_{\varepsilon}\left(x_{k}\right)$, the open ball of radius $\varepsilon$ centered at $x_{k}$. Then $Z=Z_{1} \cup \cdots \cup Z_{n}$ is a $k$-blow-up of $X$. Moreover, if we choose $Z_{k}$ as a configuration isomorphic to $Y$, then $Z$ is a $Y$-blow-up of $X$. This is always possible because any positive affine transformation preserves order type and therefore we can choose a copy of $Y$ inside any open ball.

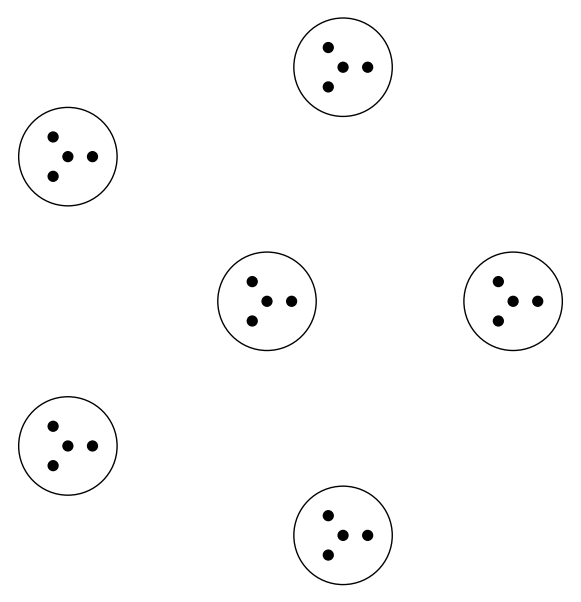

Figure 3.3: A blow-up

Figure 3.3 shows an $Y$-blow-up of $X$ when $X$ is a convex pentagon with a point in the center and $Y$ is a triangle with an interior point. Although $Y$-blow-ups of $X$ always exist for any $X$ and $Y$ in general position, they are not uniquely determined. In fact, in order to have such a blow-up $Z=Z_{1} \cup \cdots \cup Z_{n}$ we are only interested in the orientations of triples that are contained entirely in $Z_{i}$ or have intersection at most one with $Z_{i}$. Thus we are free to choose the orientation between triples that has intersection of size 2 with $Z_{i}$ and obtain non-isomorphic $Y$-blow-ups of $X$. With this in mind, define $X \otimes Y$ as the set of all order types that are $Y$-blowups of $X$. Note that an element of $X \otimes Y$ has $|X| \cdot|Y|$ points.

We extend this notation to products of more than two elements. Let $X_{1}, \ldots, X_{d} \in \mathbb{R}^{2}$ be $d$ configurations of sizes $n_{1}, \ldots, n_{d}$, respectively. We define $\bigotimes_{i=1}^{d} X_{i}$ inductively over $d$. 
If $d=2$, then $\bigotimes_{i=1}^{d} X_{i}$ is just $X_{1} \otimes X_{2}$. For $d>2$, we define $\bigotimes_{i=1}^{d} X_{i}$ as the set of order types $Y$ such that there exists a partition $Y=Y_{1} \cup \cdots \cup Y_{n_{1}}$ with $Y_{k} \in \bigotimes_{i=2}^{d} X_{i}$, for every $1 \leq k \leq n_{1}$, and such that every transversal is isomorphic to $X_{1}$. Note, by the definition, that $\bigotimes_{i=1}^{d} X_{i}$ contains $n_{1} \cdots n_{d}$ points. It turns out that those extended product are helpful to provide upper bounds for $f(B, \alpha)$.

Lemma 3.3. Let $B$ be a configuration of size $n \geq 2$ and $0<\alpha \leq 1$. Then,

$$
f(B, \alpha) \leq n^{\lceil n \log (1 / \alpha)\rceil} .
$$

Proof. Let $A \in \bigotimes_{i=1}^{d} B$ for a fixed $d$. We claim that for every $\alpha>0$ if $X$ is an $\alpha$-proportion of $A$, then $X$ contains a $\beta$-proportion of $B$ with

$$
\beta=\max \left\{1, \alpha\left(\frac{n}{n-1}\right)^{d-1}\right\} .
$$

The proof is by induction on $d$. If $d=1$, then $A \cong B$ and any $\alpha$-proportion of $A$ contains an $\alpha$-proportion of $B$. For $d>1$, let $X$ be an $\alpha$-proportion of $A$. By definition, the configuration $A$ have a partition $A=A_{1} \cup \cdots \cup A_{n}$ such that $A_{k} \in \bigotimes_{i=1}^{d-1} B$ for every $1 \leq i \leq n$ and such that every transversal is isomorphic to $B$. If $X$ contains one element of each $A_{i}$, then $X$ contains a copy of $B$ and $\beta=1$. Otherwise there exists at least one index $k$ such that $X \cap A_{k}=\emptyset$. Then by an averaging argument, there exists an index $j \neq k$ such that

$$
\left|X \cap A_{j}\right| \geq \frac{|X|}{(n-1)} \geq \frac{\alpha|A|}{n-1}=\frac{\alpha n}{n-1}\left|A_{j}\right|
$$

Therefore, $X \cap A_{j}$ is an $\frac{\alpha n}{n-1}$-proportion of $A_{j}$. Since $A_{j} \in \bigotimes_{i=1}^{d-1} B$, there exists, by induction, a subset $Y \subset X \cap A_{j} \subset X$ such that $Y$ contains a $\beta$-proportion of $B$ with

$$
\beta=\max \left\{1,\left(\frac{\alpha n}{n-1}\right) \cdot\left(\frac{n}{n-1}\right)^{d-2}\right\}=\max \left\{1, \alpha\left(\frac{n}{n-1}\right)^{d-1}\right\} .
$$

Note that the inequality $1 / t^{2}<1 / t$ holds for $t>1$. Thus, by integrating the inequality, we obtain

$$
1-\frac{1}{1+x}=\int_{1}^{1+x} \frac{1}{t^{2}} \mathrm{~d} t<\int_{1}^{1+x} \frac{1}{t} \mathrm{~d} t=\log (1+x)
$$

for $x>0$. Applying the last inequality for $x=1 /(n-1)$, we have that $\log n-\log (n-1)>1 / n$. Let $d=\lceil n \log (1 / \alpha)\rceil$. Thus, any $\alpha$-proportion of an element of $\bigotimes_{i=1}^{d} B$ contains a betaproportion of a copy of $B$ with

$$
\beta=\alpha\left(\frac{n}{n-1}\right)^{d-1}>\frac{\alpha(n-1)}{n} \cdot\left(\frac{n}{n-1}\right)^{\frac{\log (1 / \alpha)}{\log n-\log (n-1)}}=\frac{n-1}{n},
$$

which implies that it actually contains a copy of $B$. Since any configuration in $\bigotimes_{i=1}^{d} B$ has $n^{d}$ points, we obtain the desired bound. 


\subsubsection{The lower bound}

For the lower bound we use the Erdős-Szekeres theorem. Let $\operatorname{ES}(n)$ be the minimum integer $N$ such that any configuration of size $N$ in general position contains $n$ points in convex position. Erdős and Szekeres proved in [9] that $\operatorname{ES}(n)$ always exists and in [10] they proved that $\operatorname{ES}(n) \geq 2^{n-2}+1$ and conjectured this to be sharp. A recent breakthrough due to Andrew Suk [33] asymptotically solves the conjecture.

Theorem 3.4 ([33]). For $n$ sufficiently large, the following holds

$$
2^{n-2}+1 \leq \operatorname{ES}(n) \leq 2^{n+O\left(n^{2 / 3} \log n\right)}
$$

The lower bound of $\operatorname{ES}(n)$ is particularly interesting for us. It shows that there exists an order type of size $2^{n-2}$ that does not contain $n$ points in convex position. Of course, if one take a subset of this configuration it still does not contain $n$ points in convex position. Thus, there exists an order type of size $N$ that does not contain $\lceil\log N\rceil+2$ points in convex position. Let $E_{N}$ be this order type. The following proof is based on [6].

Lemma 3.5. For $n \geq 3$ and $\alpha \leq 1 / 2$ the following holds. If $B$ is a configuration in general position of size $n$, then

$$
\frac{n^{2}}{20 \alpha \log n} \leq f(B, \alpha)
$$

Proof. Let $B$ be a configuration such that $B$ is a union of two sets $B=B_{1} \cup B_{2}$ with $B_{1} \cong$ $E_{\lceil n / 2\rceil}$ and $B_{2}$ a convex $\lfloor n / 2\rfloor$-gon. There are several ways that one can combine $B_{1}$ and $B_{2}$ to form an order type $B$. This ways depends on the orientation of triples intersecting both $B_{1}$ and $B_{2}$. Choose an arbitrary combination as the order type of $B$. We claim that $B$ satisfies the statement.

Let $A$ be a configuration such that $A \rightarrow_{\alpha} B$. We construct a set $X$ by repeatedly removing convex sets of size $\lfloor n / 2\rfloor$ from $A$ and adding them to $X$. Let $X_{1} \subset A$ be a convex set of size $\lfloor n / 2\rfloor$ inside $A$. Such a set must exist because $A$ contains at least one copy of $B$. Suppose the sets $X_{1}, \ldots, X_{k}$ are already defined and now we want to define $X_{k+1}$. If the set $A \backslash\left(\bigcup_{i=1}^{k} X_{i}\right)$ contains $\lfloor n / 2\rfloor$ points in convex position, then let $X_{k+1}$ be this $\lfloor n / 2\rfloor$ points. Otherwise stop and let $X=\bigcup_{i=1}^{k} X_{i}$. Since $A$ is finite, this process eventually terminates and we end up with a set $X=X_{1} \cup \cdots \cup X_{d} \subset A$ for some integer $d$, where $X_{i}$ is a convex set of size $\lfloor n / 2\rfloor$ for every $1 \leq i \leq d$.

By the construction of $X$, the set $A \backslash X$ does not contain $\lfloor n / 2\rfloor$ points in convex position. Then $A \backslash X$ does not contain a copy of $B$ and, by hypothesis, it is less than an $\alpha$-proportion of $A$. This implies that

$$
|X|=|A|-|A \backslash X|>(1-\alpha)|A| \geq \alpha|A|,
$$

which is true for $\alpha \leq 1 / 2$. Thus $X$ is an $\alpha$-proportion and it contains a copy of $B$.

Now we do a little refinement. Let $d^{\prime}$ be the minimum integer such that $X_{1} \cup \cdots \cup X_{d^{\prime}}$ is an $\alpha$-proportion of $A$ and set $X^{\prime}=X_{1} \cup \cdots \cup X_{d^{\prime}}$. Since any $X_{i}$ has size $\lfloor n / 2\rfloor$, a calculation 
shows that

$$
\alpha|A| \leq\left|X^{\prime}\right| \leq \alpha|A|+\lfloor n / 2\rfloor \leq \alpha|A|+\frac{n}{2}=\alpha|A|+\frac{\alpha}{2} \cdot \frac{n}{\alpha} \leq \alpha|A|+\frac{\alpha}{2}|A|=\frac{3 \alpha}{2}|A|,
$$

which is true because $\alpha|A| \geq n$.

By hypothesis, $X^{\prime}$ contains a copy of $B$ and in particular a copy of $E_{\lceil n / 2\rceil}$. We claim that $\left|X_{i} \cap E_{\lceil n / 2\rceil}\right| \leq \log n+2$, for all $1 \leq i \leq d^{\prime}$ This is a consequence from the fact that any subset of a convex set is also a convex set and that the biggest convex subset in $E_{\lceil n / 2\rceil}$ has size less than $\log \lceil n / 2\rceil+2 \leq \log n+2$. Thus,

$$
\left\lceil\frac{n}{2}\right\rceil=\left|E_{\lceil n / 2\rceil}\right|=\sum_{i=1}^{d^{\prime}}\left|X_{i} \cap E_{\lceil n / 2\rceil}\right| \leq d^{\prime}(\log n+2),
$$

which implies that

$$
d^{\prime} \geq \frac{1}{\log n+2}\left\lceil\frac{n}{2}\right\rceil \text {. }
$$

Therefore we can estimate the size of $X^{\prime}$ and consequently of $A$ by

$$
|A| \geq \frac{2}{3 \alpha}\left|X^{\prime}\right|=\frac{2}{3 \alpha} d^{\prime}\left\lfloor\frac{n}{2}\right\rfloor \geq \frac{2}{3 \alpha(\log n+2)}\left\lfloor\frac{n^{2}}{4}\right\rfloor \geq \frac{n^{2}}{20 \alpha \log n}
$$

giving the desired lower bound for $n \geq 3$.

\subsubsection{A family of configuration with polynomial bounds}

Now we study a particular family of configurations that have polynomial bounds in $n$ and $1 / \alpha$. These configurations were introduced by Károlyi and Solymosi [22]. For an order type $B$ and an integer $N>0$. Let $\operatorname{ES}(B, N)$ be the maximum integer $m$ such that there exists a configuration of $m$ points in general position without a copy of $B$ and a copy of a convex $N$-gon. In their work they proved that there exist order types $B$ such that $\operatorname{ES}(B, N)=2^{N-2}$, which is of the same order of $\operatorname{ES}(N)$. However, they provided examples of $B$ such that $\operatorname{ES}(B, N)$ has polynomial bounds on $N$. These examples are special cases of the following family of order types.

Definition 3.6. Let $d \geq 1$ be an integer and $K=\left(k_{1}, \ldots, k_{d}\right), L=\left(l_{1}, \ldots, l_{d}\right)$ with $k_{i} \geq 2$, $l_{i} \geq 3$, for $1 \leq i \leq d$ be ordered sets of integers. We define the order type

$$
G_{K, L}^{d}=\left\{a_{i, j}: 1 \leq i \leq d, 1 \leq j \leq k_{i}\right\} \cup\left\{b_{i, j}: 1 \leq i \leq d, 1 \leq j \leq l_{i}\right\}
$$

as the order type such that $a_{1,1} \ldots a_{1, k_{1}} a_{2,1} \ldots a_{d, 1} \ldots a_{d, k_{d}}$ is a convex $\left(k_{1}+\cdots+k_{d}\right)$-gon listed in clockwise order, $b_{i, 1}=a_{i, k_{i}}, b_{i, l_{i}}=a_{i+1,1}$ and $b_{i, 1} \ldots b_{i, l_{i}}$ is a convex set on the interior of the triangles $a_{i, k_{i}-1} b_{i, 1} b_{i, l_{i}}$ and $b_{i, 1} b_{i, l_{i}} a_{i+1,2}$, where all indices are taken modulo $d$.

It follows from the definition that $G_{K, L}^{d}$ has $k_{1}+\cdots+k_{d}+l_{1}+\cdots+l_{d}-2 d$ points. The 
assumption that $b_{i, 1} \ldots b_{i, l_{i}}$ are inside the triangles $a_{i, k_{i}-1} b_{i, 1} b_{i, l_{i}}$ and $b_{i, 1} b_{i, l_{i}} a_{i+1,2}$ assures us that the order type of $G_{K, L}^{d}$ is unique and well defined.

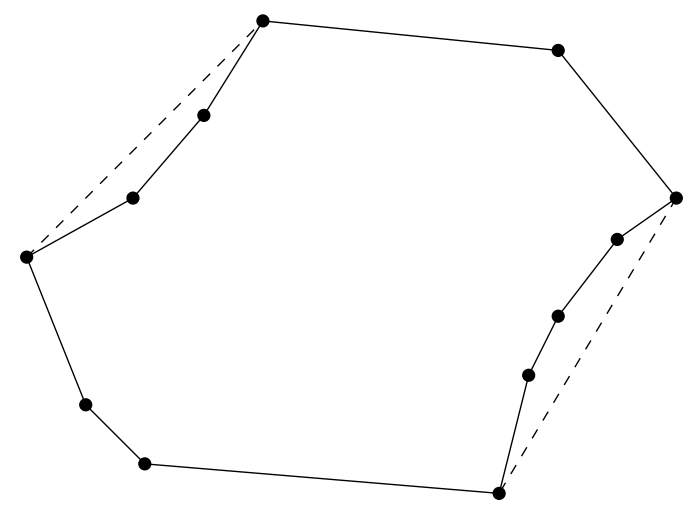

Figure 3.4: A configuration $G_{(4,3),(4,5)}^{2}$

Another important observation is that any subset of a $G_{K, L}^{d}$ is a $G_{K^{\prime}, L^{\prime}}^{d^{\prime}}$ for some $d^{\prime} \leq d$ and ordered sets $K^{\prime}, L^{\prime}$ of size $d^{\prime}$. We will prove that $f\left(G_{K, L}^{d}, \alpha\right)$ is at most quadratic in both parameters $n$ and $1 / \alpha$. In order to do that, we need the following lemma.

Lemma 3.7. Let $A=A_{1} \cup \cdots \cup A_{n}$ be a partition of a finite set $A$ such that $\left|A_{1}\right|=\cdots=\left|A_{n}\right|$ and $\alpha>0$ a real number. Then for every $X \subset A$ with $|X| \geq \alpha|A|$, there are at least $\alpha n / 2$ sets $A_{i}$ such that $\left|X \cap A_{i}\right| \geq \frac{\alpha}{2}\left|A_{i}\right|$.

Proof. Define $I=\left\{i \in[n]:\left|X \cap A_{i}\right| \geq \frac{\alpha}{2}\left|A_{i}\right|\right\}$. We want to estimate the size of $I$. This can be done by noticing that

$$
\begin{aligned}
& \alpha|A| \leq|X|=\sum_{i=1}^{n}\left|X \cap A_{i}\right|=\sum_{i \in I}\left|X \cap A_{i}\right|+\sum_{i \notin I}\left|X \cap A_{i}\right| \\
& <\sum_{i \in I}\left|A_{i}\right|+\sum_{i \notin I} \frac{\alpha\left|A_{i}\right|}{2}=\frac{|A|}{n}\left(|I|+\frac{\alpha}{2}(n-|I|)\right) .
\end{aligned}
$$

Therefore,

$$
|I|>\frac{\frac{\alpha}{2} n}{1-\frac{\alpha}{2}}>\frac{\alpha}{2} n
$$

Theorem 3.8. Let $G_{K, L}^{d}$ be the order type with parameters $d \geq 1, K=\left(k_{1}, \ldots, k_{d}\right)$, $L=\left(l_{1}, \ldots, l_{d}\right)$. Then the following holds,

$$
f\left(G_{K, L}^{d}, \alpha\right) \leq\left\lceil\frac{2 k}{\alpha}\right\rceil \cdot\left\lceil\frac{2 l}{\alpha}\right\rceil,
$$

where $k=k_{1}+\cdots+k_{d}$ and $l=\max _{1 \leq i \leq d}\left\{l_{i}\right\}$.

Proof. We will construct a configuration $A$ such that $A \rightarrow_{\alpha} G_{K, L}^{d}$. Because of the observation that subsets of order types in the family of Definition 3.6 are also in the family, it is 
natural to consider $A$ as a member of this family. In fact, let $A=G_{K_{1}, L_{1}}^{d_{1}}$ with $d_{1}=\left\lceil\frac{2 k}{\alpha}\right\rceil$, $K^{\prime}=(2,2, \ldots, 2)$ and $L^{\prime}=\left(\left\lceil\frac{2 l}{\alpha}\right\rceil,\left\lceil\frac{2 l}{\alpha}\right\rceil, \ldots,\left\lceil\frac{2 l}{\alpha}\right\rceil\right)$. One can see that $A$ has

$$
(2+\cdots+2)+\left(\left\lceil\frac{2 l}{\alpha}\right\rceil+\cdots+\left\lceil\frac{2 l}{\alpha}\right\rceil\right)-2\left\lceil\frac{2 k}{\alpha}\right\rceil=\left\lceil\frac{2 k}{\alpha}\right\rceil \cdot\left\lceil\frac{2 l}{\alpha}\right\rceil
$$

points.

We will prove that $A \rightarrow_{\alpha} B$, where $B=G_{K_{2}, L_{2}}^{d_{2}}$ with $d_{2}=k, K_{2}=(2, \ldots, 2)$ and $L_{2}=(l, \ldots, l)$. Let $A=A_{1} \cup \cdots \cup A_{d_{1}}$ be a partition of $A$ such that

$$
A_{i}=\left\{b_{i, j}: 1 \leq j \leq\left\lceil\frac{2 l}{\alpha}\right\rceil\right\}
$$

as in the Definition 3.6. This partition makes sense because every element in $K_{1}$ is 2 and therefore all the points of $A$ are contained in some $A_{i}$. Let $X$ be an $\alpha$-proportion of $A$. By Lemma 3.7, there exists at least $\frac{\alpha}{2} d_{1}$ sets $A_{i}$ with $\left|X \cap A_{i}\right| \geq \frac{\alpha}{2}\left|A_{i}\right|$. Consider $I \subset\left[d_{1}\right]$ a subset of size $k \leq \frac{\alpha}{2} d_{1}$ such that every $i \in I$ satisfies $\left|X \cap A_{i}\right| \geq \frac{\alpha}{2}\left|A_{i}\right|$ and for every $i \in I$ consider a set $B_{i} \subset A_{i} \cap X$ of size $l \leq \frac{\alpha}{2}\left|A_{i}\right|$. It is not difficult to verify that $B_{1} \cup \cdots \cup B_{k} \cong B$ and that $B \subset X$.

Now the theorem follows from the fact that $G_{K, L}^{d} \subset B$. In fact, if $B=B_{1} \cup \cdots \cup B_{k}$ is the partition described in the paragraph above, then one can find a copy of $G_{K, L}^{d}$ in the following way. Consider a partition $G_{K, L}^{d}=G_{1} \cup \cdots \cup G_{m}$ with $m \leq k$, where each $G_{t}$ is an $a_{i, j}$ with $2 \leq j \leq k_{i}$, or a $\left\{b_{i, 1} \ldots b_{i, l_{i}}\right\}$. Now embed $G_{i}$ inside $B_{i}$. Because $\left|G_{i}\right| \leq l_{i} \leq l=\left|B_{i}\right|$ and $B_{i}$ is convex this embedding is always possible and, by the definition of $B$, it is a copy of $G_{K, L}^{d}$. Therefore, $G_{K, L}^{d} \subset B$ and $A \rightarrow_{\alpha} G_{K, L}^{d}$.

The following is a quick corollary of the previous theorem.

Corollary 3.9. Let $G_{K, L}^{d}$ be an order type with $n$ points. Then,

$$
f\left(G_{K, L}^{d}, \alpha\right) \leq \frac{8 n^{2}}{\alpha^{2}}
$$

Proof. Because $G_{K, L}^{d}$ has $\left(k_{1}+\cdots+k_{d}\right)+\left(l_{1}+\cdots+l_{d}\right)-2 d=n$ and $k_{i} \geq 2, l_{j} \geq 3$, for every $1 \leq i, j \leq d$, we have that $k=k_{1}+\cdots+k_{d}<n$ and $l \leq l_{1}+\cdots+l_{n} \leq n<2 n$. Therefore, we obtain by Theorem 3.8 that

$$
f\left(G_{K, L}^{d}, \alpha\right) \leq\left\lceil\frac{2 k}{\alpha}\right\rceil \cdot\left\lceil\frac{2 l}{\alpha}\right\rceil \leq \frac{8 n^{2}}{\alpha^{2}}
$$

One can notice in Lemma 3.5 that the condition $\alpha \leq 1 / 2$ was needed. This suggests that the asymptotic behavior of some configurations may depend on the value of $\alpha$. We will show that some configurations described in Definition 3.6 has asymptotic behavior on $n$ depending on $\alpha$. 
Theorem 3.10. For every $n$ there exists a configuration $B$ of $n$ points such that

$$
f(B, \alpha)= \begin{cases}\Theta_{\alpha}(n), & \text { if } \alpha>1 / 2 \\ \Theta_{\alpha}\left(n^{3 / 2}\right), & \text { if } \alpha \leq 1 / 2\end{cases}
$$

Proof. Let $B=G_{K, L}^{d}$ be a configuration of $n$ points, where $d=\lfloor\sqrt{n}\rfloor, K=(\lfloor n / 2\rfloor+$ $2,2,2, \ldots, 2)$ and $L=\left(l_{1}, \ldots, l_{d}\right)$ with $l_{i} \in\left\{\left\lfloor\frac{\sqrt{n}}{2}\right\rfloor,\left\lfloor\frac{\sqrt{n}}{2}\right\rfloor+1\right\}$. We claim that this configuration $B$ satisfies the statement.

First notice that $B=B_{1} \cup B_{2}$ where $B_{1}=G_{K_{1}, L_{1}}^{d_{1}}$ with $d_{1}=d, K_{1}=(2, \ldots, 2), L_{1}=L$ and $B_{2}$ is a convex $\lfloor n / 2\rfloor$-gon. Suppose that $\alpha>1 / 2$ and let $\varepsilon>0$ be a real number such that $\alpha=1 / 2+\varepsilon$. Consider a configuration $A=G_{K^{\prime}, L^{\prime}}^{d^{\prime}}$ with $d^{\prime}=\left\lceil\frac{d}{\varepsilon}\right\rceil, K^{\prime}=(M, 2, \ldots, 2)$, $L^{\prime}=\left(\left\lceil\frac{\sqrt{n}}{\varepsilon}\right\rceil, \ldots,\left\lceil\frac{\sqrt{n}}{\varepsilon}\right\rceil\right)$ and $M=\left\lceil\frac{d}{\varepsilon}\right\rceil \cdot\left\lceil\frac{\sqrt{n}}{\varepsilon}\right\rceil$. We can also notice that $A=A_{1} \cup A_{2}$ where $A_{1}=G_{K_{1}^{\prime}, L_{1}^{\prime}}^{d^{\prime}}$ with $d_{1}^{\prime}=d^{\prime}, K_{1}^{\prime}=(2, \ldots, 2), L_{1}^{\prime}=L^{\prime}$ and $A_{2}$ is a convex $M$-gon. We will prove now that $A \rightarrow_{\alpha} B$.

Let $X$ be an $\alpha$-proportion of $A$ and $X_{1}=X \cap A_{1}, X_{2}=X \cap A_{2}$. Because $\left|A_{1}\right|=\left|A_{2}\right|$ it follows that $\left|X_{1}\right| \geq \varepsilon\left|A_{1}\right|$. In fact,

$$
\alpha|A|=\left(\frac{1}{2}+\varepsilon\right)|A| \leq|X|=\left|X_{1}\right|+\left|X_{2}\right| \leq\left|X_{1}\right|+\frac{1}{2}|A| .
$$

Therefore $\left|X_{1}\right| \geq \varepsilon|A| \geq 2 \varepsilon\left|A_{1}\right|$. In a similar way we can also prove that $\left|X_{2}\right| \geq 2 \varepsilon\left|A_{2}\right|$. Now as in the proof of Theorem 3.8 a $2 \varepsilon$-proportion of $A_{1}$ contains a $G_{K_{2}^{\prime}, L_{2}^{\prime}}^{d^{\prime}}$ with $d_{2}^{\prime}=\varepsilon d_{1} \geq d$, $K_{2}^{\prime}=(2, \ldots, 2)$ and $L_{2}^{\prime}=(\lfloor\sqrt{n}\rfloor, \ldots,\lfloor\sqrt{n}\rfloor)$. However, because $\left\lfloor\frac{\sqrt{n}}{2}\right\rfloor+1 \leq\lfloor\sqrt{n}\rfloor$, we have that $B_{1} \subset G_{K_{2}^{\prime}, L_{2}^{\prime}}^{d^{\prime}} \subset X_{1}$. A $2 \varepsilon$-proportion of $A_{2}$ contains a convex set of size $2 \varepsilon M \geq \frac{n}{\varepsilon} \geq$ $\lfloor n / 2\rfloor$ and then $B_{2} \subset X_{2}$. Using the uniqueness of Definition 3.6, we obtain that $B \subset X$.

Computing the number of points of $A$ we obtain

$$
f(B, \alpha) \leq|A|=2 M \leq \frac{4 n}{\varepsilon^{2}}=\frac{4 n}{(\alpha-1 / 2)^{2}} .
$$

Using the trivial lower bound made in the introduction of this chapter we obtain that $f(B, \alpha) \geq n / \alpha$ and $f(B, \alpha)=\Theta_{\alpha}(n)$.

If $\alpha<1 / 2$, then we can apply Theorem 3.8 to obtain that

$$
f(B, \alpha) \leq\left\lceil\frac{2 k}{\alpha}\right\rceil \cdot\left\lceil\frac{2 l}{\alpha}\right\rceil .
$$

Using that $k=k_{1}+\cdots+k_{d}=\lfloor n / 2\rfloor+2(\lfloor\sqrt{n}\rfloor+1)<n$ and $l=\max _{1 \leq i \leq d} l_{i}=\left\lfloor\frac{\sqrt{n}}{2}\right\rfloor+1<\sqrt{n}$ we have that

$$
f(B, \alpha) \leq \frac{4 n^{3 / 2}}{\alpha^{2}} .
$$

For the lower bound we use exactly the same proof as that in Lemma 3.5. The only difference here is that $B_{1}$ is not the order type provided by the Erdős-Szekeres construction. Instead $B_{1}=G_{K_{1}, L_{1}}^{d_{1}}$ as described above. It is possible to see that $B_{1}$ does not contain a 
convex set of size $2 \sqrt{n}$. This happens because in order to construct a convex set we can either pick at most two vertices from each $b_{i, 1} \ldots b_{i, l_{i}}$, or pick vertices of only one $b_{i, 1} \ldots b_{i, l_{i}}$. Now consider $A$ such that $A \rightarrow_{\alpha} B$ and an $\alpha$-proportion $X^{\prime}$ consisting of the union of $d^{\prime}$ convex $\lfloor n / 2\rfloor$-gons $X_{1}, \ldots, X_{d^{\prime}}$ exactly as in Lemma 3.5. Because $B \subset X^{\prime}$, we have that $B_{1} \subset X^{\prime}$. Therefore,

$$
\left\lceil\frac{n}{2}\right\rceil=\left|B_{1}\right|=\sum_{i=1}^{d^{\prime}}\left|X_{i} \cap B_{1}\right| \leq 2 d^{\prime} \sqrt{n}
$$

and we have

$$
d^{\prime} \geq \frac{n^{1 / 2}}{4}
$$

Using now that $X^{\prime}$ was constructed such that $|A| \geq \frac{3}{2 \alpha}\left|X^{\prime}\right|$ we conclude that

$$
|A| \geq \frac{2}{3 \alpha}\left|X^{\prime}\right|=\frac{2 d^{\prime}}{3 \alpha}\left\lfloor\frac{n}{2}\right\rfloor \geq \frac{n^{3 / 2}}{15 \alpha},
$$

which proves that $f(B, \alpha)=\Theta_{\alpha}\left(n^{3 / 2}\right)$.

\subsection{Extremal number for the grid}

For a configuration $B$ of $n$ points and a real number $\alpha>0$ define

$$
N_{0}(B, \alpha)=\min \left\{N \in \mathbb{Z}:[N]^{2} \rightarrow_{\alpha} B\right\}
$$

as the minimum integer $N$ such that an $\alpha$-proportion of $[N]^{2}$ contains a copy of $B$. As in the definition of $f(B, \alpha)$ we can also define

$$
N_{0}(n, \alpha)=\max \left\{N_{0}(B, \alpha): B \text { is an order type of size } n\right\}
$$

as the maximum over all values of $N_{0}(B, \alpha)$ for $|B|=n$. First we notice that the problem of finding bounds on $\operatorname{ex}(N, B)$ is closely related to finding bounds on $N_{0}(B, \alpha)$. Indeed, if $N \geq N_{0}(B, \alpha)$, then by the definition we have that $[N]^{2} \rightarrow_{\alpha} B$. Thus we can define a inverse function $\alpha_{0}(N, B)$ as the smallest real number $\alpha_{0}>0$ such that $N \geq N_{0}\left(B, \alpha_{0}\right)$. Because any $\alpha_{0}$-proportion of $[N]^{2}$ contains a copy of $B$, this gives the natural bound $\operatorname{ex}(N, B) \leq \alpha_{0} N^{2}$.

For instance, suppose that for a certain configuration $B$ we obtain the bound

$$
N_{0}(B, \alpha) \leq \frac{n^{2}}{\alpha^{3}} .
$$


Thus in order to obtain a bound for $\operatorname{ex}(N, B)$ we could use $\alpha_{0}$ such that

$$
N \geq \frac{n^{2}}{\alpha_{0}^{3}} \geq N_{0}\left(B, \alpha_{0}\right)
$$

This gives us

$$
\alpha_{0} \geq \frac{n^{2 / 3}}{N^{1 / 3}}
$$

and taking $\alpha_{0}=\frac{n^{2 / 3}}{N^{1 / 3}}$ we obtain that

$$
\operatorname{ex}(N, B) \leq \alpha_{0} N^{2}=n^{2 / 3} N^{5 / 3}
$$

One can derive lower bounds for $\operatorname{ex}(N, B)$ from lower bounds of $N_{0}(B, \alpha)$ in exactly the same way done above. For this reason we will focus our attention for the rest of this section on the $N_{0}(B, \alpha)$ problem. As we shall see, this formulation of the problem is close to the $f(B, \alpha)$ problem, which will be helpful later.

We start by noticing that one can give bounds on $N_{0}$ using the multidimensional Szemerédi theorem.

Theorem 3.11. ([12], [17], [24]) For all $l \geq 2, d \geq 1$ and $\alpha \geq 0$. There exists $N_{0}=$ $N_{0}(l, r, \alpha)$ such that if $X \subset[N]^{r}$ with $N \geq N_{0}$ and $|X| \geq \alpha N^{r}$, then $X$ contains a homothetic copy of $[l]^{r}$, i.e., a set of the form $z+j[l]^{r}$ for some $z \in[N]^{r}$ and $j>0$.

In our context, Theorem 3.11 basically states that for every $N \geq N_{0}(\operatorname{gr}(B), 2, \alpha)$ any $\alpha$-proportion of $[N]^{2}$ contains a homothetic copy of $[\operatorname{gr}(B)]^{2}$. Because $[\operatorname{gr}(B)]^{2}$ contains a copy of $B$ and homothety preserves order types, we obtain that $N_{0}(B, \alpha) \leq N_{0}(\operatorname{gr}(B), 2, \alpha)$.

Theorem 3.11 was first proved by Furstenberg and Katznelson in [12]. Their proof was based on arguments in ergodic theory and gives no bounds on $N_{0}$. Later, quantitatives proofs were given by Gowers ([17]) and Nagle, Rödl, Schacht and Skokan ([28], [24]), independently. These proofs rely on regularity lemmas for hypergraphs and because of that they provide non-practical bounds on $N_{0}$ and consequently on $N_{0}(B, \alpha)$. For instance, Gowers showed in [16] a bound for $r=1$ that is an exponential tower of height 6 .

Our main intention in this section is to give an upper bound for $N_{0}(B, \alpha)$ that avoids the multidimensional Szemerédi theorem. This is done by creating a relation between the $f(B, \alpha)$ problem and the problem of embedding configuration in a grid of minimum size.

\subsubsection{Averaging lemma}

Our proof of Theorem 1.1 strongly relies on the open property of order types (Proposition 2.4). Basically, we are not looking for a homothetic copies of a given configuration as in Theorem 3.11, but for a homothetic $\varepsilon$-perturbation of a given configuration. This difference enables us to find upper bounds for $N_{0}$ using constructions in the real plane. The following lemma is crucial to realize these ideas. 
Lemma 3.12. Let $r>0$ be an integer and $X \subset[N]^{r}$ with $|X| \geq \alpha N^{r}$. For any configuration $A \subset[N]^{r}$, there exists an $z \in \mathbb{Z}^{r}$ such that $|(A+z) \cap X| \geq \frac{\alpha}{2^{r}}|A|$.

In other words, Lemma 3.12 says that for a configuration $A \in[N]^{2}$ and an $\alpha$-proportion $X$, there exists a translation of $A$, that is, a copy of $A$ in $[N]^{2}$ such that $X$ is an $\alpha / 4$ proportion of this copy. Figure 3.5 exemplifies the content of the lemma.

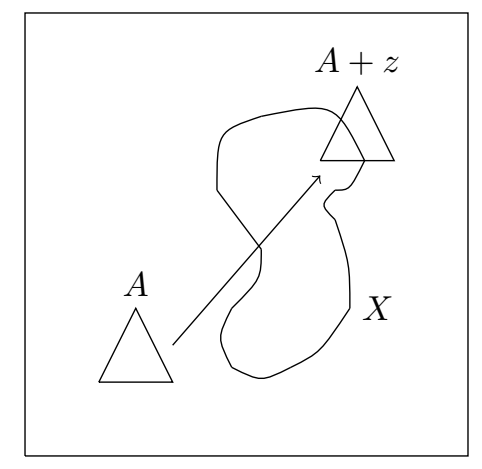

Figure 3.5: Averaging Lemma

As we can see there is a translation $A+z$ of $A$ such that the intersection of $X$ with $A+z$ is relatively big. The proof of this lemma is just an application of the averaging method.

Proof of Lemma 3.12. Consider the set $Z=[-(N-1), N]^{r} \cap \mathbb{Z}^{r}$. We will choose $z \in Z$ uniformly at random and estimate the size of $|(A+z) \cap X|$. Let $W$ be the random variable counting the size of $|(A+z) \cap X|$. By the linearity of expectation we have

$$
\mathbb{E}(W)=\sum_{x \in X} \mathbb{P}(x \in(A+z))
$$

For every point $x \in X$, there are exactly $|A|$ elements $z \in Z$ such that $x \in A+z$. In fact, $x \in(A+z)$ implies that $z \in x-A$ and because $x-A$ has size $|A|$ and is a subset of $Z$ the result follows. This means that $\mathbb{P}(x \in(A+z))=\frac{|A|}{|Z|}$ for every $x \in X$. Therefore,

$$
\mathbb{E}(W)=\sum_{x \in X} \frac{|A|}{|Z|}=\frac{|X| \cdot|A|}{|Z|} \geq \frac{\alpha N^{r}|A|}{(2 N)^{r}}=\frac{\alpha}{2^{r}}|A|
$$

and, by the definition of expectation, there exists a $z \in Z$ such that $|(A+z) \cap X| \geq \mathbb{E}(W) \geq$ $\frac{\alpha}{2^{r}}|A|$.

Lemma 3.12 allows us to give bounds on $N_{0}$ depending on configurations $A$ related to the density problem in the plane.

Theorem 3.13. Let $B$ be a configuration and $\alpha>0$. If $A$ is a configuration such that $A \rightarrow_{\alpha / 4} B$, then $N_{0}(B, \alpha) \leq \operatorname{gr}(A)$.

Proof. Let $N=\operatorname{gr}(A)$ and $X$ be an $\alpha$-proportion of $[N]^{2}$. Thus, by Lemma 3.12 there exists a copy of $A$ such that $X \cap A$ is an $\alpha / 4$-proportion of $A$. Using that $A \rightarrow_{\alpha / 4} B$ we have that $B \subset X \cap A \subset X$ and $[N]^{2} \rightarrow_{\alpha} B$, which finishes the proof. 
The last theorem gives us a method to obtain lower bounds on $N_{0}$. First we obtain a configuration in the plane such that $A \rightarrow_{\alpha / 4} B$ and then we embed this configuration in a grid $\operatorname{gr}(A)$. The problem to find small configurations $A$ in the plane was already studied in the previous section. Now we will focus on embedding these configurations in reasonably grids.

\subsubsection{Blow-up embedding}

In this subsection we will study bounds for embedding a configuration in the grid. In particular the configuration that we are interested in is one such that $A \rightarrow_{\alpha / 4} B$. In Lemma 3.3 we saw that the configurations $A \in \bigotimes_{i=1}^{d} B$ are good candidates. One possible approach is to use Theorem 2.18 to obtain an upper bound for $\operatorname{gr}(A)$ based upon the size of $|A|$.

Consider the configuration $A \in \bigotimes_{i=1}^{d} B$, with $d=\lceil n \log (4 / \alpha)\rceil$. Lemma 3.3 assures us that $A \rightarrow_{\alpha / 4} B$ and then $N_{0}(B, \alpha) \leq \operatorname{gr}(A)$. To estimate the size of the grid we use Theorem 2.18, which gives $\operatorname{gr}(A) \leq 2^{2^{c|A|}}$ for a fixed constant $c>0$. Since $A \in \bigotimes_{i=1}^{d} B$ has $n^{d}$ points, we obtain that

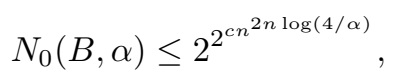

for sufficiently large $n$. This already gives a mouch better upper bound comparing to the bound by the Szemerédi's theorem. However, we can further improve it. Notice that configuration $A$ arises after iterated blow-up constructions over $B$. If we can emulate this contruction on the grid, then it is expected that we can bound $\operatorname{gr}(A)$ by $\operatorname{gr}(B)$. We will prove that this is indeed the case.

Lemma 3.14. Given configurations $X$ and $Y$, there exists a configuration $A \in X \otimes Y$ satisfying the following conditions

(i) $\operatorname{gr}(A) \leq 8 \operatorname{gr}(X)^{2} \operatorname{gr}(Y)$,

(ii) Every $\frac{1}{2 \operatorname{gr}(Y) \sqrt{2}}$-perturbation of $A$ is in $X \otimes Y$.

Proof. Lemma 2.10 will be of great importance. By the definition of $\operatorname{gr}(X)$, there exists some copy of $X$ inside the $[\operatorname{gr}(X)]^{2}$ grid. For simplicity assume that $X$ is exactly this copy. The open property assures us that there exists $\varepsilon$ such that every $\varepsilon$-perturbation of $X$ is isomorphic to $X$. Moreover, because $X$ is embedded on a grid, we have $\Delta_{\min }(X) \geq 1 / 2$. Thus by Lemma 2.10 with $L=\operatorname{gr}(X)-1$, every $\left(\frac{1}{2 \sqrt{2} \operatorname{gr}(X)}\right)$-perturbation of $X$ is isomorphic to $X$.

For an integer $k>0$, consider a homothety that dilates $X$ to a copy $X^{\prime}$ which is $k$ times greater than $X$. It is easy to see that $X^{\prime}$ can be embedded in a $[k \cdot \operatorname{gr}(X)]^{2}$ grid and that every $\left(\frac{k}{2 \sqrt{2} \operatorname{gr}(X)}\right)$-perturbation of $X^{\prime}$ is isomorphic to $X$. Let $X^{\prime}=\left\{x_{1}, \ldots, x_{n}\right\}$ and for every $x_{i} \in X^{\prime}$ let $X_{i}$ be the open ball centered at $x_{i}$ of radius $\frac{k}{2 \sqrt{2} \operatorname{gr}(X)}$. We know that for every configuration $Z=\left\{z_{1}, \ldots, z_{n}\right\}$ with $z_{i} \in X_{i}$, the congruence $Z \cong X$ holds. Our task is to embed a copy $Y_{i}$ of $Y$ inside each $X_{i} \cap[0, L]^{2}$ (We are assuming that the grid is inside 


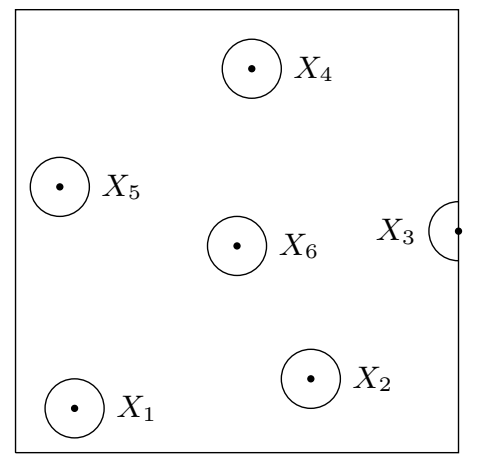

Figure 3.6: $X$ and its open balls

the $[0, L]^{2}$ square). Then by the definition of blow-up, the configuration $A=Y_{1} \cup \cdots \cup Y_{n}$ will be an element of $X \otimes Y$.

To embed a copy of $Y$ inside each $X_{i}$, each $X_{i} \cap[0, L]^{2}$ must contain a grid of size at least $\operatorname{gr}(Y)$. Because the point $x_{i}$ could be on the border of $[0, L]^{2}$, the region $X_{i} \cap[0, L]^{2}$ could be in the worst case a quarter of a circle. By Pythagoras theorem, every quadrant of an open circle of radius $r$ centered at a point of the grid contains a grid of size $\frac{r \sqrt{2}}{2}$. Thus each $X_{i} \cap[0, L]^{2}$ contains a grid of size at least $\frac{k}{4 \operatorname{gr}(X)}$. Taking $k=8 \operatorname{gr}(X) \operatorname{gr}(Y)$, each $X_{i} \cap[0, L]^{2}$ contains a grid of size $2 \operatorname{gr}(Y)$. Since $2 \operatorname{gr}(Y) \geq \operatorname{gr}(Y)+2$, it is possible to embed a copy $Y_{i}$ of $Y$ in each $X_{i}$ in a way that every 1-perturbation of this copy is inside $X_{i}$. In particular, every $\frac{1}{2 \operatorname{gr}(Y) \sqrt{2}}$-perturbation $Z_{i}$ of $Y_{i}$ is inside $X_{i}$. By Lemma 2.10, we have that $Z_{i} \cong Y$ and we conclude that $Z_{1} \cup \cdots \cup Z_{n} \in X \otimes Y$. Thus, we obtained an element $A \in X \otimes Y$ inside a grid of size $k \cdot \operatorname{gr}(X)=8 \operatorname{gr}(X)^{2} \operatorname{gr}(Y)$ such that every $\frac{1}{2 \operatorname{gr}(Y) \sqrt{2}}$-perturbation is in $X \otimes Y$.

The condition (ii) in the lemma will be important later in Section 4.2. Iterated applications of Lemma 3.14 provides the following result.

Theorem 3.15. Given a configuration $X$ and an integer $d \geq 1$, there exists a configuration $A \in \bigotimes_{i=1}^{d} X$ satisfying the following conditions

(i) $\operatorname{gr}(A) \leq 8^{d-1} \operatorname{gr}(X)^{2 d-1}$,

(ii) Every $\frac{1}{2 \operatorname{gr}(X) \sqrt{2}}$-perturbation of $A$ is in $\bigotimes_{i=1}^{d} X$.

Proof. The proof is by induction on $d$. For $d=1$, the statement is true. Now suppose that the theorem is true for every $k<d$. Let $A^{\prime} \in \bigotimes_{i=1}^{d-1} X$ be a configuration satisfying the statement for $d-1$. By Lemma 3.14, there exists a configuration $A \in \bigotimes_{i=1}^{d} X$ such that

$$
\operatorname{gr}(A) \leq 8 \operatorname{gr}(X)^{2} \operatorname{gr}\left(A^{\prime}\right) \leq 8 \operatorname{gr}(X)^{2} \cdot 8^{d-2} \operatorname{gr}(X)^{2 d-3}=8^{d-1} \operatorname{gr}(X)^{2 d-1} .
$$

The lemma also says, if we read the proof, that there exists a partition $A=A_{1} \cup \cdots \cup A_{n}$ such that every $A_{i} \cong A^{\prime}$ and such that the following holds. If $Z=Z_{1} \cup \cdots \cup Z_{n}$ is an 1-perturbation of $A$ such that $Z_{i}$ is an 1-perturbation of $A_{i}$, then every transversal of $Z$ is isomorphic to $X$. In particular, taking a $\frac{1}{2 g(X) \sqrt{2}}$-perturbation $Z=Z_{1} \cup \cdots \cup Z_{n}$ of $A$ 
we obtain by the induction hypothesis that every $Z_{i} \in \bigotimes_{i=1}^{d-1} X$ and every transversal is isomorphic to $X$. Therefore, $Z$ is an element of $\bigotimes_{i=1}^{d} X$.

\subsubsection{Extremal number estimates}

Theorem 3.15 allows us to obtain an element $A \in \bigotimes_{i=1}^{d} B$ with $\operatorname{gr}(A)$ bounded by $\operatorname{gr}(B)$. This is enough to give the following general bound.

Theorem 3.16. Given $\varepsilon>0$, there exists $n_{0}$ such that for every configuration $B$ with $n \geq n_{0}$ points the following holds,

$$
\frac{1}{\sqrt{\alpha}}(\operatorname{gr}(B)-1) \leq N_{0}(B, \alpha) \leq(3 \operatorname{gr}(B))^{3 n \log (4 / \alpha)} .
$$

Proof. The lower bound follows from the observation that a grid of $\operatorname{size} \operatorname{gr}(B)-1$ do not contain a copy of $B$ and thus can not be an $\alpha$-proportion of $\left[N_{0}\right]^{2}$. This gives

$$
(\operatorname{gr}(B)-1)^{2} \leq \alpha N_{0}^{2}
$$

and thus,

$$
N_{0} \geq \frac{1}{\sqrt{\alpha}}(\operatorname{gr}(B)-1)
$$

For the upper bound we just have to combine all the results obtained in this section so far. By Theorem 3.13 we have $N_{0}(B, \alpha) \leq \operatorname{gr}(A)$ for $A$ such that $A \rightarrow_{\alpha / 4} B$. Choose $d=\lceil n \log (4 / \alpha)\rceil$. By Lemma 3.3 we have that $A \rightarrow_{\alpha / 4} B$ for every $A \in \bigotimes_{i=1}^{d} B$. Therefore, by Theorem 3.15 , we have that

$$
N_{0}(B, \alpha) \leq \operatorname{gr}(A) \leq 8^{d-1} \operatorname{gr}(B)^{2 d-1} \leq(3 \operatorname{gr}(B))^{2 d} \leq(3 \operatorname{gr}(B))^{3 n \log (4 / \alpha)} .
$$

An immediate consquence of the last theorem is the following corollary.

Corollary 3.17. There exist $c_{1}, c_{2}>0$ such that

$$
2^{2^{c_{1} n}+\frac{1}{2} \log (1 / \alpha)} \leq N_{0}(n, \alpha) \leq 2^{2^{c_{2} n} \log (1 / \alpha)}
$$

Proof. By Theorem 3.16 we have that

$$
\frac{1}{\sqrt{\alpha}}(\operatorname{gr}(n)-1) \leq N_{0}(n, \alpha) \leq(3 \operatorname{gr}(n))^{3 n \log (4 / \alpha)},
$$

and the result follows using Theorem 2.18.

Now we proceed to obtain bounds on $\operatorname{ex}(N, B)$. As outlined previously, our approach is based on viewing the extremal problem as the inverse problem of the density problem. 
Proof of Theorem 1.1. Fix an integer $N$. We are interested in finding $\alpha_{0}$ such that

$$
N \geq(3 \operatorname{gr}(B))^{3 n \log \left(4 / \alpha_{0}\right)} \geq N_{0}\left(B, \alpha_{0}\right) .
$$

So we have

$$
\alpha_{0} \geq 4 \exp \left(-\frac{\log N}{3 n \log (3 \operatorname{gr}(B))}\right)=4 N^{-\eta}
$$

Taking $\alpha_{0}=4 N^{-\eta}$ we obtain that $[N]^{2} \rightarrow_{\alpha_{0}} B$ and therefore $\operatorname{ex}(N, B) \leq \alpha_{0} N^{2}=4 N^{2-\eta}$.

It is possible to give lower bounds for $\operatorname{ex}(N, B)$. The lower bound given in Theorem 3.16 can be translated as $\operatorname{ex}(N, B) \geq(\operatorname{gr}(B)-1)^{2}$. This lower bound does not use the parameter $N$. Another possible lower bound is the following one. Consider a grid $[N]^{2}$. One can see that the set consisting of the first $(n / 2-1)$ rows does not contain a configuration of $n$ points in general position. This is a consequence of the pigeonhole principle. Therefore, because $B$ is a configuration in general position, $N_{0}$ has to satisfy the inequality $\alpha N_{0}^{2} \geq$ $(n / 2-1) N_{0}$, that is, $N_{0} \geq \frac{n / 2-1}{\alpha}$. Translating this to the extremal number problem, we obtain $\operatorname{ex}(N, B) \geq(n / 2-1) N$. However, we did not use anything about the order type $B$. It would be interesting to find a lower bound that uses both parameters (see Chapter 5).

\subsubsection{Convex case}

Although the previous approach gives a general bound, we would like to study the case where $B$ is a convex set. In this case it is possible to give a much better bound for $f(B, \alpha)$. Just notice that every subset of a convex set is also a convex set. As we saw in the introduction, $f(B, \alpha)=\left\lceil\frac{n}{\alpha}\right\rceil$ and this is attainable by a convex set. This suggests that, in order to estimate $N_{0}(B, \alpha)$, it is probably much better to take $A$, in Theorem 3.13, as a convex set than as a blowup of $B$. The next result confirms this observation. It is important to state that a more precise result was proved by Jarník [21].

Theorem 3.18. If $B$ is a convex set of size $n$, then $\operatorname{gr}(B)=\Theta\left(n^{3 / 2}\right)$.

Proof. Suppose $B$ can be embedded in a grid of size $N$. Let $B=\left\{\left(x_{i}, y_{i}\right): 1 \leq x_{i}, y_{i} \leq\right.$ $N, \forall 1 \leq i \leq n\}$ be the coordinates of each point in the $[N]^{2}$ grid in a way that the points $\left(x_{1}, y_{1}\right), \ldots,\left(x_{n}, y_{n}\right)$ are in counterclockwise order. We define $s_{i}=\frac{y_{i+1}-y_{i}}{x_{i+1}-x_{i}}$ as the slope of the line between points $\left(x_{i}, y_{i}\right)$ and $\left(x_{i+1}, y_{i+1}\right)$. Of course, the indices are taken modulo $n$ and if $x_{i}=x_{i+1}$ we define

$$
s_{i}= \begin{cases}\infty, & \text { if } y_{i+1}-y_{i}>0 \\ 0, & \text { if } y_{i+1}-y_{i}=0 \\ -\infty, & \text { if } y_{i+1}-y_{i}<0\end{cases}
$$

For an index $i$, define $\theta_{i}$ as the measure of the angle of the slope $s_{i}$, i.e., $\theta_{i}$ is the angle formed by the lines $\left(x_{i}, y_{i}\right)\left(N+1, y_{i}\right)$ and $\left(x_{i}, y_{i}\right)\left(x_{i+1}, y_{i+1}\right)$ in counterclockwise direction. We can 
divide the indices into 8 classes depending on where $\theta_{i}$ belongs. Let $T_{k}=\left\{i \in[n]: \frac{(k-1) \pi}{4}<\right.$ $\left.\theta_{i} \leq \frac{k \pi}{4}\right\}$, for $1 \leq k \leq 8$. Clearly $[n]=T_{1} \cup \cdots \cup T_{k}$ is a partition, then there exists an index $i$ such that $\left|T_{i}\right| \geq n / 8$. Because $B$ is convex, we get that $T_{k}$ consists of consecutive elements of $[n]$ and thus, by symmetry, we can suppose without loss of generality that $T_{1}=[t]$ with $t \geq n / 8$.

The convexity of $B$ and the definition of $T_{1}$ ensure that $0<s_{1}<\cdots<s_{t} \leq 1$. Our task is to estimate the minimum size of a grid containing $B_{1}=\left\{\left(x_{i}, y_{i}\right): i \in[t+1]\right\} \subset$ $B$, this will give a bound on $\operatorname{gr}(B)$. The minimum square grid containing $B_{1}$ has size $\max \left\{x_{t+1}-x_{1}, y_{t+1}-y_{1}\right\}+1$. Because $\frac{y_{i+1}-y_{i}}{x_{i+1}-x_{i}}=s_{i} \leq 1$ we have,

$$
y_{t+1}-y_{1}=\sum_{i=1}^{t}\left(y_{i+1}-y_{i}\right) \leq \sum_{i=1}^{t}\left(x_{i+1}-x_{i}\right)=x_{t+1}-x_{1},
$$

and then $\max \left\{x_{t+1}-x_{1}, y_{t+1}-y_{1}\right\}+1=x_{t+1}-x_{1}+1$. For each $i$, write $s_{i}=\frac{a_{i}}{b_{i}}$ with $a_{i}, b_{i}$ nonnegative integers such that $\operatorname{gcd}\left(a_{i}, b_{i}\right)=1$. This means that $y_{i+1}-y_{i}=k a_{i}$ and $x_{i+1}-x_{i}=k b_{i}$ for some positive integer $k$, then it follows that $x_{i+1}-x_{i} \geq b_{i}$ and

$$
x_{t+1}-x_{1}+1=\sum_{i=1}^{t-1}\left(x_{i+1}-x_{i}\right)+1 \geq b_{1}+\cdots+b_{t}+1 .
$$

Therefore, estimating $b_{1}+\cdots+b_{t}$ is enough to give a lower bound on $\operatorname{gr}(B)$.

For a fixed integer $s$, the number of different fractions $\frac{r}{s}$ with $0<\frac{r}{s} \leq 1$ and $\operatorname{gcd}(r, s)=1$ is $\varphi(s)$ (See Appendix A). It is not hard to see that in order to minimize the sum $\sum_{i=1}^{t} b_{i}$, we have to take the fractions $\left\{\frac{r}{s}: \operatorname{gcd}(r, s)=1,0<r \leq s, 1 \leq s \leq x\right\}$ as the slopes for an appropriate value of $x$. The value of $x$ must satisfy $\sum_{s \leq x} \varphi(s) \leq t$ and the sum of the denominators of this fractions is $\sum_{s \leq x} s \varphi(s)$. Theorem A.2 gives the right estimates to proceed. Indeed, for sufficiently large $n$ and $x=\frac{\pi \sqrt{t}}{2}$ we have

$$
\sum_{s \leq x} \varphi(s)=\frac{3}{\pi^{2}} x^{2}+O(x \log x) \leq \frac{4}{\pi^{2}} x^{2}=t
$$

Thus,

$$
\operatorname{gr}(B)>b_{1}+\cdots+b_{t} \geq \sum_{s \leq x} s \varphi(s)=\frac{2}{\pi^{2}} x^{3}+O\left(x^{2} \log x\right) \geq \frac{1}{\pi^{2}} x^{3}=\frac{\pi t \sqrt{t}}{8}>\frac{\pi n^{3 / 2}}{256}
$$

The upper bound uses the same argument. Let $q$ be the smallest integer such that $h=\sum_{s \leq q} \varphi(s) \geq n-1$. We will construct a convex set of size $h+1$. For this consider the set of fractions $S=\left\{\frac{a}{b}: \operatorname{gcd}(a, b)=1,0<a \leq b, 1 \leq b \leq q\right\}$ and let $s_{1}<\cdots<s_{h}$ be an ordering of $S$. Denote $s_{i}=\frac{a_{i}}{b_{i}}$ with $\operatorname{gcd}\left(a_{i}, b_{i}\right)=1$. Set $z_{1}=(1,1)$ and $z_{i+1}=z_{i}+\left(b_{i}, a_{i}\right)$, for every $1 \leq i \leq h$. The set $\left\{z_{1}, \ldots, z_{h+1}\right\}$ is a convex set of size $h+1 \geq n$ inside a grid of size $1+b_{1}+\cdots+b_{h}$.

Now we do an estimate similar to the lower bound. By Theorem A.2, for sufficiently 
large $n$ we have

$$
h=\frac{3}{\pi^{2}} q^{2}+O(q \log q) \geq \frac{2}{\pi^{2}} q^{2},
$$

and

$$
q \leq \frac{\pi \sqrt{h}}{\sqrt{2}}
$$

Finally,

$$
\operatorname{gr}(B) \leq 1+\sum_{i=1}^{h} b_{i}=1+\sum_{s \leq q} s \varphi(s)=1+\frac{2}{\pi^{2}} q^{3}+O\left(q^{2} \log q\right) \leq \frac{3}{\pi^{2}} q^{3} \leq \frac{3 \pi h \sqrt{h}}{2 \sqrt{2}} \leq 3 \pi n^{3 / 2} .
$$

Where we use that $h=\varphi(q)+\sum_{s \leq q-1} \varphi(s) \leq \varphi(n)+n<2 n$.

Combining the last theorem with Theorem 3.13 for $A$ as a convex $\left\lfloor\frac{n}{\alpha}\right\rceil$-gon and the lower bound provided by Theorem 3.16 we obtain that

$$
c_{1} \frac{n^{3 / 2}}{\sqrt{\alpha}} \leq N_{0}(B, \alpha) \leq c_{2} \frac{n^{3 / 2}}{\alpha \sqrt{\alpha}}
$$

for $B$ convex, $c_{1}$ and $c_{2}$ constants and $n$ sufficiently large. Therefore, for convex sets $N_{0}(B, \alpha)=\Theta\left(n^{3 / 2}\right)$. Translating this bound on $N_{0}(B, \alpha)$ to a bound on $\operatorname{ex}(N, B)$ we obtain that $\operatorname{ex}(N, B) \leq c n N^{4 / 3}$ for some constant $c$. Unfortunately, this is not sharp on $N$. The reason why this is true, is because good bounds on $\operatorname{ex}(N, B)$ in terms of $N$ depends on good bounds on $N_{0}(B, \alpha)$ as a function of $\alpha$ and not as a function of $n$.

Theorem 3.19. If $B$ is a convex set of $n$ points, then $\operatorname{ex}(N, B)=\Theta_{n}(N)$.

Proof. We claim that for every positive integer $k$, there exists a configuration $A$ of $k$ points in general position such that $\operatorname{gr}(A) \leq 2 k$. First we show that for $p$ prime, there exists a configuration $A$ of $p-1$ points such that $\operatorname{gr}(A) \leq p-1$. Consider the multiplicative group $\mathbb{Z}_{p}^{\times}$. Standard results in algebra says that $\mathbb{Z}_{p}^{\times}$is cyclic and has at least one generator $g \in \mathbb{Z}_{p}$. The set $A=\left\{\left(g^{i}, g^{2 i}\right): 1 \leq i \leq p-1\right\} \subset[p-1]^{2}$ is our natural candidate. Here we are considering $g^{i}$ as $g^{i}$ (mod. $p$ ) if we think of $g$ as an element of $\mathbb{Z}$. For any three points $x=\left(g^{i}, g^{2 i}\right), y=\left(g^{j}, g^{2 j}\right)$ and $z=\left(g^{t}, g^{2 t}\right)$ it follows that

$$
\left.[x y z] \equiv\left|\begin{array}{lll}
g^{i} & g^{2 i} & 1 \\
g^{j} & g^{2 j} & 1 \\
g^{t} & g^{2 t} & 1
\end{array}\right| \equiv\left(g^{t}-g^{j}\right)\left(g^{t}-g^{i}\right)\left(g^{j}-g^{i}\right) \not \equiv 0 \text { (mod. } p\right) \text {. }
$$

This means that $[x y z] \neq 0$ and consequently this three points are not collinear. Thus $A$ is in general position and is inside a grid of size $p-1$. For general $k$, Bertrand postulate says that there always exists a prime number between $k$ and $2 k$. Then we can find a $k<p \leq 2 k$ such that there exists a configuration $A$ of $p-1$ points in general position with $\operatorname{gr}(A) \leq p-1 \leq 2 k$. In particular, there exists a configuration $A^{\prime}$ of $k$ points such that $\operatorname{gr}\left(A^{\prime}\right) \leq 2 k$. 
The idea for the upper bound is to give another bound on $N_{0}(B, \alpha)$, one that is better in the parameter $\alpha$. We will achieve this by finding a configuration $A$ such that $A \rightarrow_{\alpha / 4} B$ and $\operatorname{gr}(A)$ is linear. By the Erdős-Szekeres theorem, it is reasonable to try configurations in general position. Let $A$ be a configuration in general position with $|A|=\left\lceil\frac{2^{2 n+2}}{\alpha}\right\rceil$ points. Every $\frac{\alpha}{4}$-proportion $X$ of $A$ contains at least $2^{2 n}$ points and by Theorem 3.4, we have that $X$ contains a convex set of size $n$. This means that $A \rightarrow_{\alpha / 4} B$ for every $A$ in general position of size $\left\lceil\frac{2^{2 n+2}}{\alpha}\right\rceil$. The claim in the last paragraph says that there exists such $A$ in general position with $\operatorname{gr}(A) \leq 2\left\lceil\frac{2^{2 n+2}}{\alpha}\right\rceil$. Then by Theorem 3.13 we have

$$
N_{0}(B, \alpha) \leq \operatorname{gr}(A) \leq 2\left\lceil\frac{2^{2 n+2}}{\alpha}\right\rceil \leq \frac{2^{2 n+4}}{\alpha}
$$

For a fixed integer $N>0$, pick $\alpha_{0}=\frac{2^{2 n+4}}{N}$. Then

$$
N_{0}\left(B, \alpha_{0}\right) \leq \frac{2^{2 n+4}}{\alpha_{0}}=N
$$

and $[N]^{2} \rightarrow_{\alpha_{0}} B$. Therefore $\operatorname{ex}(N, B) \leq \alpha_{0} N^{2}=2^{2 n+4} N$. For the lower bound we use the observation that $\operatorname{ex}(N, B) \geq(n / 2-1) N$. 


\section{Chapter 4}

\section{Probabilistic Results}

In this chapter we study probabilistic results concerning order types. We are interested in a particular random process already described in the introduction and in Subsection 2.1.1. This process of choosing $n$ random points in the unit square can be viewed as the most natural way to generate an order type in the plane. Mainly because the random process of choosing independently every orientation does not work well since there are choices of orientations that are not realizable in the euclidean plane.

Of course, the very standard question is to determine the probability of obtaining a certain order type by this process. For instance, in the convex case Valtr showed the following result.

Theorem 4.1 ([34]). The set of $n$ points chosen independently and uniformly from the unit square is convex with probability

$$
\left(\frac{\left(\begin{array}{c}
2 n-2 \\
n-1
\end{array}\right)}{n !}\right)^{2}
$$

In Section 4.1 we will provide bounds for the general case. Although these bounds are far from optimal, they are good enough to determine the threshold of the $n$-universal property.

In Section 4.2 we deal with another problem. Given a configuration $B$ of $n$ points, what is the probability of $B$ being a subest of a $N$-random? Turns out that this probability gets smaller as $N$ tends to the infinity. As we shall see, by understanding this probabilities, we can obtain strong bounds on the probability of hereditary properties.

\subsection{Threshold for the $n$-universal property}

We start with a definition.

Definition 4.2. A configuration $X$ is n-universal if $X$ contains all order types of size $n$

Figure 4.1 exemplifies a 4-universal configuration. Indeed, the order types of size 4 are the triangle with an interior point and the convex quadrilateral. Both are included in this 


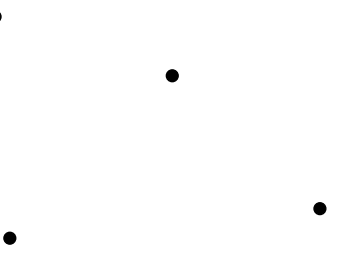

Figure 4.1: 4-universal configuration

configuration. Define

$$
R(n)=\min \{|A|: A \text { is } n \text {-universal }\}
$$

as the minimum size of an $n$-universal configuration. Determine good bounds on $R(n)$ seems to be a hard problem (see Chapter 5). However, the probabilistic version of this problem is simpler. Our aim in this section is to give a proof of Theorem 1.3, which basically says how many points we have to choose in our random process in order to obtain an $n$-universal set almost surely.

In order to prove this result, we have to give bounds for the probability of obtaining a fixed order type. One possible approach is to use Lemma 2.5 and discretize the problem of finding a probability in the unit square, to finding the probability in the integer grid. However, here we have a more elegant, and perhaps insightful, way of computing this probability. For any configuration $X$, we denote $\Delta_{\max }(X), \Delta_{\min }(X)$ as the areas of the largest and smallest triangles in $X$, respectively.

Definition 4.3. Given a configuration $X$, we define

$$
\nu(X)=\inf \left\{\frac{\Delta_{\max }(Y)}{\Delta_{\min }(Y)}: Y \cong X\right\},
$$

as the infimum of the ratios between the areas of the largest and smallest triangles for every configuration $Y$ isomorphic to $X$.

The parameter $\nu$ is well defined and $\nu(X) \geq 1$, for every configuration $X$. For instance, if $X$ is a convex quadrilateral we can show that $\nu(X)=1$ and if $X$ is a triangle with an interior point, then $\nu(X)=3$. Given an order type $B$ and a random set $U$, both of $n$ points, our aim is to estimate $\mathbb{P}(U \cong B)$ using this new parameter $\nu(B)$. Later we will find a relation between $\nu(B)$ and $\operatorname{gr}(B)$. This will provide bounds that depend only on $\operatorname{gr}(B)$.

\subsubsection{The probability of a given order type}

We divide the proof into two parts. The following is a well-known result in probabilistic combinatorics. 
Proposition 4.4. Let $A, B, C$ be three random points chosen independently inside the unit square $[0,1]^{2}$ and let $s>0$ be a real number. If $\Delta:=\Delta(A B C)$ is the area of triangle $A B C$, then the following holds,

$$
\mathbb{P}(\Delta \leq s) \leq 16 \pi s
$$

Proof. Let $b$ be the distance from $A$ to $B$. For any small $h$, we have

$$
\mathbb{P}(x \leq b \leq x+h) \leq \pi(x+h)^{2}-\pi x^{2}=\pi\left(2 x h+h^{2}\right)
$$

and taking $h \rightarrow 0$, we obtain $\mathbb{P}(x \leq b \leq x+\mathrm{d} x)=2 \pi x \mathrm{~d} x$. Now suppose that the distance between $A$ and $B$ is $x$. Then in order to have $\Delta \leq s$, the altitude of $C$ in relation to $A B$ must be at most $\frac{2 s}{x}$. This means that $C$ lies on a strip of width $\frac{4 s}{x}$ and lenght at most the diagonal of $[0,1]^{2}$. As $0 \leq x \leq \sqrt{2}$, we have

$$
\mathbb{P}(\Delta \leq s) \leq \int_{0}^{\sqrt{2}}\left(\frac{4 s \sqrt{2}}{x}\right)(2 \pi x) \mathrm{d} x=16 \pi s .
$$

Proposition 4.4 can be used to give an upper bound.

Lemma 4.5. Let $U$ be the configuration obtained by choosing $n$ points at random inside the unit square $[0,1]^{2}$. Then for any configuration $B$ of $n$ points the following holds,

$$
\mathbb{P}(U \cong B) \leq \frac{8 \pi}{\nu(B)}\left(\begin{array}{l}
n \\
3
\end{array}\right) .
$$

Proof. Let $A$ be any configuration isomorphic to $B$ inside $[0,1]^{2}$. By the definition of $\nu(B)$ and the fact that a triangle inside the unit square has area at most $1 / 2$, it follows

$$
\nu(B) \leq \frac{\Delta_{\max }(A)}{\Delta_{\min }(A)} \leq \frac{1}{2 \Delta_{\min }(A)}
$$

and we obtain that

$$
\Delta_{\min }(A) \leq \frac{1}{2 \nu(B)}
$$

This means that any configuration isomorphic to $B$ inside $[0,1]^{2}$ has a triangle with area at most $\frac{1}{2 \nu(B)}$. Proposition 4.4 says that the probability of 3 random points having area at most $\frac{1}{2 \nu(B)}$ is bounded by $\frac{8 \pi}{\nu(B)}$. Then by the union bound,

$$
\mathbb{P}(U \cong B) \leq \mathbb{P}\left(\Delta_{\min }(U) \leq \frac{1}{2 \nu(B)}\right) \leq\left(\begin{array}{l}
n \\
3
\end{array}\right) \frac{8 \pi}{\nu(B)} .
$$

The next lemma deals with the lower bound. 
Lemma 4.6. Let $U$ be the configuration obtained by choosing $n$ points at random inside the unit square $[0,1]^{2}$. Then for any configuration $B$ of $n$ points the following holds,

$$
\mathbb{P}(U \cong B) \geq \frac{n ! \pi}{512^{n} \nu(B)^{2 n}} .
$$

Proof. Let $A$ be a configuration isomorphic to $B$ such that $\frac{\Delta_{\max }(A)}{\Delta_{\min }(A)} \leq 2 \nu(B)$. This configuration exists because of the definition of $\nu(B)$. We claim that there exists a positive affine transformation $A^{\prime}$ of $A$ inside $[0,1]^{2}$ such that $\Delta_{\max }\left(A^{\prime}\right) \geq 1 / 4$. Let $P_{A}, Q_{A}$ be two points of $A$ that are at distance $\operatorname{diam}(A)$, that is, two points at the maximum possible distance. Consider the positive affine transformation $T$ that sends $A$ to $X=T(A)$ and $P_{A}, Q_{A}$ to the points $P=(0,1 / 2)$ and $Q=(1,1 / 2)$. Because of the choice of $P, Q$ we have that $X$ is inside the two lines determined by $x=0$ and $x=1$.

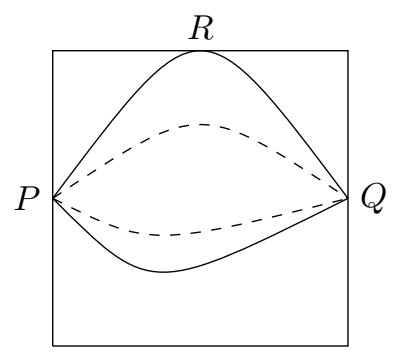

Figure 4.2: Stretching of $X$

There are two possibilities. The first one is that $X$ is not entirely inside $[0,1]^{2}$. In this case there exists a point $R \in X$ such that the distance of $R$ and the line $P Q$ is greater than $1 / 2$. However, this implies that the area of $P Q R$ is at least $1 / 4$. By a proper translation of $X$ we obtain a set $A^{\prime}$ such that $\Delta_{\max }\left(A^{\prime}\right) \geq 1 / 4$. The second case is when $X$ is inside $[0,1]^{2}$. Then we consider an affine transformation $(x, y) \mapsto\left(x, \lambda\left(y-\frac{1}{2}\right)+\frac{1}{2}\right)$ for an appropriate $\lambda>0$. This affine transformation will stretch $X$ until some point touches one of the horizontal sides of $[0,1]^{2}$, while keeping the segment $P Q$ fixed (Figure 4.2). Let $R$ be the point touching a side of $[0,1]^{2}$ and $A^{\prime}$ be the final set. The triangle $P Q R$ has area $1 / 4$ and therefore $\Delta_{\max }\left(A^{\prime}\right) \geq 1 / 4$.

The important point about positive affine transformations is that they preserve ratio about areas and order type (Proposition 2.12). Therefore, we have found a configuration $A^{\prime}$ isomorphic to $B$ such that

$$
2 \nu(B) \geq \frac{\Delta_{\max }(A)}{\Delta_{\min }(A)}=\frac{\Delta_{\max }\left(A^{\prime}\right)}{\Delta_{\min }\left(A^{\prime}\right)} \geq \frac{1}{4 \Delta_{\min }\left(A^{\prime}\right)},
$$

that gives

$$
\Delta_{\min }\left(A^{\prime}\right) \geq \frac{1}{8 \nu(B)} .
$$

This bound on the minimum area of $A^{\prime}$ allows us to apply Lemma 2.10 and obtain a region 
with positive area such that all configurations are isomorphic to $B$. Write $A^{\prime}=\left\{a_{1}, \ldots, a_{n}\right\}$ and let $A_{i}^{\prime}$ be the intersection of the open ball centered at $a_{i}$ of radius $\frac{1}{8 \nu(B) \sqrt{2}}$ and $[0,1]^{2}$. Lemma 2.10 says that every $\left(\frac{1}{8 \nu(B) \sqrt{2}}\right)$-perturbation of $A^{\prime}$ is isomorphic to $A^{\prime}$. Therefore, every transversal of $A_{1}^{\prime} \cup \cdots \cup A_{n}^{\prime}$ is isomorphic to $B$, that is, if $Y=\left\{y_{1}, \ldots, y_{n}\right\}$ is such that $y_{i} \in A_{i}^{\prime}$ for every $i$, then $Y \cong A^{\prime} \cong B$. Thus, we can bound the probability by

$$
\mathbb{P}(U \cong B) \geq \mathbb{P}\left(U \text { is a traversal of } A_{1}^{\prime} \cup \cdots \cup A_{n}^{\prime}\right)=n ! \mu\left(A_{1}^{\prime}\right) \ldots \mu\left(A_{n}^{\prime}\right)
$$

Because some points of $A^{\prime}$ can be on the border of $[0,1]^{2}$ we can only assure that $\mu\left(A_{i}^{\prime}\right) \geq$ $\frac{\pi}{4}\left(\frac{1}{8 \nu(B) \sqrt{2}}\right)^{2}$ and it follows that,

$$
\mathbb{P}(U \cong B) \geq n !\left(\frac{\pi}{512 \nu(B)^{2}}\right)^{n}=\frac{n ! \pi^{n}}{512^{n} \nu(B)^{2 n}}
$$

\subsubsection{Relationship between $\nu(B)$ and $\operatorname{gr}(B)$}

The two lemmas in the previous subsection give upper and lower bounds on $P(U \cong B)$ in terms of $\nu(B)$. However we still do not know much about this parameter $\nu(B)$. The next lemma shows that this parameter is closely related to $\operatorname{gr}(B)$, the minimum size of a grid containing a copy of $B$.

Lemma 4.7. For any configuration $B$ the following holds,

$$
\frac{\operatorname{gr}(B)}{10} \leq \nu(B) \leq \operatorname{gr}(B)^{2}
$$

Proof. By the definition of $\operatorname{gr}(B)$, there exists a copy of $B$ inside $[\operatorname{gr}(B)]^{2}$. Let $A$ be this copy. Because every point of $A$ has integral coordinates, we have $\Delta_{\min }(A) \geq 1 / 2$. On the other hand, every triangle inside $[\operatorname{gr}(B)]^{2}$ has area at most half of the area of $[\operatorname{gr}(B)]^{2}$. Thus

$$
\nu(B) \leq \frac{\Delta_{\max }(A)}{\Delta_{\min }(A)} \leq \operatorname{gr}(B)^{2} .
$$

For the lower bound consider the configuration $A^{\prime}$ as in Lemma 4.6 , i.e., a configuration $A^{\prime} \cong B$ such that $A^{\prime} \subset[0,1]^{2}, \Delta_{\min }(A) \geq \frac{1}{8 \nu(B)}$. A homothety of ratio $N=\lceil 8 \nu(B)\rceil$ centered at the origin sends $A^{\prime}$ to a configuration $X \cong B$ such that $X \subset[0, N]^{2}, \Delta_{\min }(X) \geq$ $\frac{N^{2}}{8 \nu(B)}$. Now the argument is similar to the one in the proof of Lemma 2.25. Inside the $[0, N]^{2}$ there exists a grid of size $N+1$ and every point inside $[0, N]^{2}$ is at distance at most $\frac{\sqrt{2}}{2}$ to a point of this grid. Lemma 2.10 says that every $\left(\frac{N}{8 \nu(B) \sqrt{2}}\right)$-perturbation of $X$ is isomorphic to $B$ and a simple computation shows that

$$
\frac{N}{8 \nu(B) \sqrt{2}}=\frac{\lceil 8 \nu(B)\rceil}{8 \nu(B) \sqrt{2}} \geq \frac{\sqrt{2}}{2}
$$

This means that there exists a configuration $Y$ that is isomorphic to $B$ and have integral 
coordinates. Therefore, we obtain

$$
\operatorname{gr}(B) \leq N+1=\lceil 8 \nu(B)\rceil+1 \leq 8 \nu(B)+2 \leq 10 \nu(B)
$$

where we use that $\nu(B) \geq 1$.

Combining the results so far, we obtain the following bound on $\mathbb{P}(U \cong B)$.

Corollary 4.8. Let $U$ be the configuration obtained by choosing $n$ points at random inside the unit square $[0,1]^{2}$. Then for any configuration $B$ of $n$ points the following holds,

$$
\frac{n ! \pi^{n}}{512^{n} \operatorname{gr}(B)^{4 n}} \leq \mathbb{P}(U \cong B) \leq\left(\begin{array}{l}
n \\
3
\end{array}\right) \frac{80 \pi}{\operatorname{gr}(B)} .
$$

This result is far from being sharp. The gap between the upper and lower bound can be noticed in the convex case. Indeed, Theorem 3.18 gives that $\operatorname{gr}(B) \leq 3 \pi n^{3 / 2}$ in this case and thus by Corollary 4.8 we obtain $\mathbb{P}(U \cong B) \leq c n^{3 / 2}$ for some constant $c$. This do not provide any information since $c n^{3 / 2}$ is much larger than the obvious bound $\mathbb{P}(U \cong B) \leq 1$. However the last corollary can be useful if $\operatorname{gr}(B)$ grows much above exponential. For instance, this is the case for the configuration $B$ constructed in Subsection 2.3.1. Lemmas 2.23 ad 2.25 show that for this configuration there exist $c_{1}, c_{2}>0$ constants such that

$$
2^{2^{c_{1} n}} \leq \operatorname{gr}(B) \leq 2^{2^{c_{2} n}}
$$

Therefore, another corollary of our work is the following.

Corollary 4.9. There exist constants $c_{1}, c_{2}>0$ such that the following holds. For every $n$ sufficiently large, there exists an order type $B$ of $n$ points such that

$$
2^{-2^{c_{1} n}} \leq \mathbb{P}(U \cong B) \leq 2^{-2^{c_{2} n}}
$$

where $U$ is the random set obtained by choosing $n$ points independently inside the unit square.

\subsubsection{Proof of Theorem 1.3}

Now we give a proof of the $n$-universal treshold theorem. The proof is a simple application of the second moment method and of Corollary 4.8 .

Proof of Theorem 1.3. Theorem 2.18 gives us constants $b_{1}$ and $b_{2}$ such that

$$
2^{2^{b_{1} n}} \leq \operatorname{gr}(n) \leq 2^{2^{b_{2} n}}
$$

for every $n$ sufficiently large. Let $c_{1}=b_{1} / 3$ and $c_{2}=3 b_{2}$. Let $U$ be the random set obtained by choosing $N$ points uniformly and independently inside the unit square. First we prove the 0 -statement, that is, we prove that if $N \leq 2^{2^{c_{1} n}}$, then $\lim _{n \rightarrow \infty} \mathbb{P}(U$ is $n$-universal $)=0$. 
For every $n$ sufficiently large, let $B_{n}$ be the configuration of $n$ points of Subsection 2.3.1 such that $\operatorname{gr}(B) \geq 2^{2^{b_{1} n}}$. By Corollary 4.8 , the probability of an $n$-random set being isomorphic to $B_{n}$ is

$$
p_{B_{n}}=\mathbb{P}\left(V \cong B_{n}\right) \leq 2^{-2^{b_{1} n / 2}} .
$$

Let $X_{B_{n}}$ be the random variable counting the number of copies of $B_{n}$ inside $U$. By linearity of expectation, we have

$$
\mathbb{E}\left(X_{B_{n}}\right)=\sum_{S \subset U,|S|=n} \mathbb{P}\left(S \cong B_{n}\right)=\left(\begin{array}{c}
N \\
n
\end{array}\right) p_{B_{n}} .
$$

Markov's inequality gives us that

$$
\mathbb{P}\left(X_{B_{n}}>0\right) \leq \mathbb{E}\left(X_{B_{n}}\right) \leq N^{n} p_{B_{n}} \leq 2^{n \cdot 2^{c_{1} n}} \cdot 2^{-2^{b_{1} n / 2}} \leq 2^{-2^{c_{1} n}} \rightarrow 0,
$$

as $n \rightarrow \infty$. Because a $n$-universal configuration must contain a copy of $B_{n}$, we obtain that

$$
\lim _{n \rightarrow \infty} \mathbb{P}(U \text { is } n \text {-universal }) \leq \lim _{n \rightarrow \infty} \mathbb{P}\left(X_{B_{n}}>0\right)=0
$$

For the 1-statement we will use the second moment. We suppose now that $N \geq 2^{2^{c_{2} n}}$. Let $B$ be an order type of $n$ points and $X_{B}$ be the random variable counting the number of copies of $B$ in $U$. A union bound shows that,

$$
\mathbb{P}(U \text { is not } n \text {-universal }) \leq \sum_{|B|=n} \mathbb{P}\left(X_{B}=0\right),
$$

where the right hand sum runs over all order types $B$ of size $n$. Thus if $\sum_{|B|=n} \mathbb{P}\left(X_{B}=0\right)$ is small, then with high probability $U$ is $n$-universal. Fix a configuration $B$. A standard variant of the Chebyshev's inequality says that

$$
\mathbb{P}\left(X_{B}=0\right) \leq \frac{\operatorname{Var}\left(X_{B}\right)}{\mathbb{E}\left(X_{B}\right)^{2}} .
$$

Luckily enough, the assumption on $N$ being double exponential allows us to compute the variance of $X_{B}$ with no effort. For a subset $S \subset U$, let $X_{B, S}$ denote the indicator function 
of $S \cong B$. Indeed, a simple computation shows that

$$
\begin{aligned}
& \operatorname{Var}\left(X_{B}\right)=\sum_{\substack{S, T \subset U \\
|S|=|T|=n}} \operatorname{Cov}\left(X_{B, S}, X_{B, T}\right) \leq \\
& \sum_{\substack{S, T \subset U \\
|S|=|T|=n}}\left(\mathbb{E}\left(X_{B, S} \cdot X_{B, T}\right)-\mathbb{E}\left(X_{B, S}\right) \mathbb{E}\left(X_{B, T}\right)\right) \leq \\
& \sum_{\substack{S, T \subset U \\
|S|=|T|=n \\
|S \cap T| \neq 0 \\
n}} \mathbb{P}\left(X_{B, S}=X_{B, T}=1\right) \leq \sum_{\substack{S, T \subset U \\
|S|=|T|=n \\
|S \cap T| \neq 0}} \mathbb{P}(S \cong B)= \\
& \sum_{\substack{S=1 \\
S, T \subset U \\
|S|=|T|=n \\
|S \cap T|=i}} \mathbb{P}(S \cong B) \leq p_{B}\left(\sum_{i=1}^{N^{2 n-i}}\right) \leq n N^{2 n-1} p_{B},
\end{aligned}
$$

where we use that the probability of sets $S$ and $T$ are isomorphic to $B$ is less or equal than the probability of only $S$ is isomorphic to $B$. Because $\operatorname{gr}(B) \leq 2^{2^{b_{2} n}}$, by Corollary 4.8 , we have that

$$
p_{B}=\mathbb{P}(V \cong B) \geq 2^{-2^{2 b_{2} n}}
$$

for $n$ sufficiently large. Thus, for every configuration $B$, we have

$$
\begin{aligned}
& \mathbb{P}\left(X_{B}=0\right) \leq \frac{\operatorname{Var}\left(X_{B}\right)}{\mathbb{E}\left(X_{B}\right)^{2}} \leq \frac{n N^{2 n-1} p_{B}}{\left.\left(\begin{array}{l}
N \\
n
\end{array}\right) p_{B}\right)^{2}} \\
& \leq \frac{n^{2 n+1}}{N p_{B}} \leq \frac{n^{2 n+1}}{2^{2^{c_{2}}} \cdot 2^{-2^{2 b_{2} n}}} \leq \frac{n^{2 n+1}}{2^{2^{b_{2}}}} .
\end{aligned}
$$

Finally, using Theorem 2.3 we obtain

$$
\mathbb{P}(U \text { is not } n \text {-universal }) \leq \sum_{|B|=n} \mathbb{P}\left(X_{B}=0\right) \leq \frac{n^{2 n+1}}{2^{2^{b^{2}}}} \cdot 2^{4 n \log n+O(n)} \rightarrow 0,
$$

as $n \rightarrow \infty$. Therefore $\lim _{n \rightarrow \infty} \mathbb{P}(U$ is $n$-universal $)=1$.

\subsection{Probability of not containing an order type}

In this section we will prove Theorem 1.4. The proof will be divided into several steps. First we will reduce the problem of computing the probability of not containing an order type to a counting problem in the grid (Proposition 4.10). This will be a consequence of Lemma 2.5. Then we will give bounds to this counting problem by using the recent results of Balogh, Morris, Samotij, Saxton, Thomasson [2], [29] on independents set in hypergraphs. Finally, we will apply these results to obtain bounds to the probability of satisfying a fixed hereditary property (Corollary 4.16). 


\subsubsection{Discretization of the probability}

Let us remind the notation, let $U$ be a $N$-random set obtained by choosing $N$ points uniformly and independently at random inside the unit square $[0,1]^{2}$. Similarly, define $V_{m}$ as the random set obtained by doing the same process, but now inside the $[\mathrm{m}]^{2}$ grid.

Given a configuration $B$ and integers $N, m$, let

$$
\mathcal{N}_{m, N}(B)=\left\{A \subset[m]^{2}:|A|=N, B \not \subset A\right\},
$$

be the set of actual $N$-subsets of $[m]^{2}$ without a copy of $B$. Also let

$$
\mathcal{C}_{m, N}(B)=\left\{A \subset\left([m]^{2}\right)^{N}: B \not \subset A\right\}
$$

be the set of $N$-tuples without a copy of $B$. The difference between these two sets is that the second one allows repetition and permutation of the points, while the first one $A$ is a set with distinct points.

Proposition 4.10. Let $B$ be a configuration with $n$ points and $U$ be a $N$-random set inside the unit square. Then we have,

$$
\mathbb{P}(B \not \subset U)=\lim _{m \rightarrow \infty} \frac{N !\left|\mathcal{N}_{m, N}(B)\right|}{m^{2 N}} .
$$

Proof. For a fixed configuration $B$ of $n<N$ points we define

$$
\mathcal{B}_{N}=\{A:|A|=N, B \not \subset A\}
$$

as the order types $A$, not necessarily in general position, such that $A$ does not contain a copy of $B$. This means that if $B \not \subset U$, then $U \in \mathcal{B}_{N}$. Therefore, by Lemma 2.5 and by the fact that $\mathcal{B}_{N}$ is finite, we have that

$$
\mathbb{P}(B \not \subset U)=\sum_{A \in \mathcal{B}_{N}} \mathbb{P}(A \cong U)=\sum_{A \in \mathcal{B}_{N}} \lim _{m \rightarrow \infty} \mathbb{P}\left(A \cong V_{m}\right)=\lim _{m \rightarrow \infty} \mathbb{P}\left(B \not \subset V_{m}\right) .
$$

and we just need to calculate $\mathbb{P}\left(B \not \subset V_{m}\right)$.

By the definition of $V_{m}$ we have,

$$
\mathbb{P}\left(B \not \subset V_{m}\right)=\frac{\left|\mathcal{C}_{m, N}(B)\right|}{m^{2 N}} .
$$

We can estimate the size of $\mathcal{C}_{m, N}(B)$ via $\mathcal{N}_{m, N}(B)$ by noticing that the $N$-tuples of $\mathcal{C}_{m, N}(B)$ can be divided into two classes. The first class is of the $N$-tuples which all entries are different. This class consists of all permutations of $N$-sets inside $[m]^{2}$, that is exactly the sets in $\mathcal{N}_{m, N}(B)$. The second part is of the $N$-tuple which some of the entries are equal. We estimate this part by noticing that every $N$-tuple has at leas two entries with the same element of $[m]^{2}$. Therefore, it has at most $\left(\begin{array}{c}N \\ 2\end{array}\right) m^{2}$ choices for this pair and $\left(m^{2}\right)^{N-2}$ for the 
rest. Then,

$$
N !\left|\mathcal{N}_{m, N}(B)\right| \leq\left|\mathcal{C}_{m, N}(B)\right| \leq N !\left|\mathcal{N}_{m, N}(B)\right|+\left(\begin{array}{c}
N \\
2
\end{array}\right) m^{2 N-2}
$$

Dividing everything by $m^{2 N}$ we obtain

$$
\frac{N !\left|\mathcal{N}_{m, N}(B)\right|}{m^{2 N}} \leq \mathbb{P}\left(B \not \subset V_{m}\right) \leq \frac{N !\left|\mathcal{N}_{m, N}(B)\right|+\left(\begin{array}{c}
N \\
2
\end{array}\right) m^{2 N-2}}{m^{2 N}}
$$

and the result follows by letting $m$ goes to infinty,

$$
\mathbb{P}(B \not \subset U)=\lim _{m \rightarrow \infty} \mathbb{P}\left(B \not \subset V_{m}\right)=\lim _{m \rightarrow \infty} \frac{N !\left|\mathcal{N}_{m, N}(B)\right|}{m^{2 N}} .
$$

This proposition says that in order to obtain the desired probability we have to estimate $\mathcal{N}_{m, N}(B)$, that is, the number of subsets of $[m]^{2}$ of size $N$ that do not contain a copy of $B$. Let $\mathcal{H}$ be the $n$-graph with $V(\mathcal{H})=[m]^{2}$ and $E(\mathcal{H})=\left\{A \subset[m]^{2}: A \cong B\right\}$. In this interpretation, a subset that does not contain a copy of $B$ is a independent set and $\left|\mathcal{N}_{m, N}(B)\right|$ is exactly the number of independent sets of size $N$ in $\mathcal{H}$. The problem of counting the number of independent sets of a fixed size in a hypergraph can be solved using the container method. The technical details of the method is the content of the next subsection.

\subsubsection{Hypergraph containers}

Now we present the result of hypergraph containers. The technique, developed independently by Saxton and Thomason ([29]) and Balogh, Morris and Samotij ([2]), says that independent sets of a balanced uniform hypergraph are clustered in a small number of containers. This is of great interest when we are counting such independent sets, because we can do that by estimating only in a small number of sets (containers). We will use the notation in [2]. First, we start with an important definition. A family $\mathcal{F}$ of sets of vertices is increasing if for every $A, B \subset V, A \in \mathcal{F}$ and $A \subset B$ imply that $B \in \mathcal{F}$.

Definition 4.11. Let $\mathcal{H}$ be an $r$-graph, let $\mathcal{F}$ be an increasing family of subsets of $V(\mathcal{H})$ and let $\varepsilon \in(0,1)$. We say that $\mathcal{H}$ is $(\mathcal{F}, \varepsilon)$-dense if

$$
e(\mathcal{H}[A]) \geq \varepsilon e(\mathcal{H})
$$

for every $A \in \mathcal{F}$.

There always exists a family $\mathcal{F} \in \mathcal{P}(V(\mathcal{H}))$ such that $\mathcal{H}$ is $(\mathcal{F}, \varepsilon)$-dense. For instance, consider the family

$$
\mathcal{F}=\{A \subset V(\mathcal{H}): e(\mathcal{H}[A]) \geq \varepsilon e(\mathcal{H})\}
$$


In applications of the container theorem, we are interested in increasing families $\mathcal{F}$ defined as $\mathcal{F}=\{A \in V(\mathcal{H}):|A| \geq \delta v(\mathcal{H})\}$ for some appropriate $\delta>0$. This is mainly because the theorem will guarantee that our containers are in the complement $\overline{\mathcal{F}}$. Therefore, with this choice of $\mathcal{F}$, our containers will be sets of size at most $\delta v(\mathcal{H})$. However, now we have to prove that $\mathcal{H}$ is $(\mathcal{F}, \varepsilon)$-dense. As we shall see, this is usually done with a supersaturation theorem.

Given an $r$-graph $\mathcal{H}$, denote by $d_{\mathcal{H}}(A)=|\{e \in E(\mathcal{H}): A \subset e\}|$ the number of edges that contain a subset $A \subset V(\mathcal{H})$. Then we can define

$$
\Delta_{k}(\mathcal{H})=\max \left\{d_{\mathcal{H}}(A):|A|=k\right\}
$$

A balanced $r$-graph means that there exists some $c>0$ and $p \in(0,1)$ such that

$$
\Delta_{k}(\mathcal{H}) \leq c p^{k-1} \frac{e(\mathcal{H})}{v(\mathcal{H})}, \quad \forall 1 \leq k \leq r
$$

Let $\mathcal{I}$ be the set of independent sets of a balanced $r$-graph $\mathcal{H}$ and let $\mathcal{F}$ be a family such that $\mathcal{H}$ is $(\mathcal{F}, \varepsilon)$-dense. The container theorem will assign a container $C \in \overline{\mathcal{F}}$ for each independent set $I \in \mathcal{I}$ such that $I \subset C$. For technical reasons that are interesting to us, this is done in the following way: Given a set $I$, there exists a small set $S(I) \subset I$, that is called the fingerprint of $I$ and for each fingerprint $S$ there is a container $C(S)$. This is done in a way that $S(I) \subset I \subset C(S(I))$. Since each fingerprint is small, the number of containers can be bounded in a good way.

Theorem 4.12 ([2]). For every $r \in \mathbb{N}$ and all positive $c$ and $\varepsilon$, there exists a positive constant $K$ such that the following holds. Let $\mathcal{H}$ be an $r$-graph and let $\mathcal{F} \subset \mathcal{P}(V(\mathcal{H})$ ) be an increasing family of sets such that $|A| \geq \varepsilon v(\mathcal{H})$ for all $A \in \mathcal{F}$. Suppose that $\mathcal{H}$ is $(\mathcal{F}, \varepsilon)$-dense and there exists $p \in(0,1)$ such that, for every $1 \leq k \leq r$,

$$
\Delta_{k}(\mathcal{H}) \leq c p^{k-1} \frac{e(\mathcal{H})}{v(\mathcal{H})}
$$

Then there exist a family $\mathcal{S} \subset \mathcal{P}(V(\mathcal{H}))$ and functions $f: \mathcal{I} \rightarrow \mathcal{S}$ and $g: \mathcal{S} \rightarrow \overline{\mathcal{F}}$ such that

(i) $f(I) \leq K p v(\mathcal{H})$, for every $I \in \mathcal{I}$.

(ii) $f(I) \subset I \subset g(f(I))$, for every $I \in \mathcal{I}$.

Moreover, the constant $K:=K(r, c, \varepsilon)$ can be taken as $K=K_{1} \varepsilon^{-r} \log (1 / \varepsilon)$ for some constant $K_{1}:=K_{1}(r, c)$ depending only on $c$ and $r$.

One important observation is that the constant $K$ has no relation with the size of the hypergraph $\mathcal{H}$. Thus the theorem is true even for small hypergraphs satisfying the hypothesis.

We want to apply Theorem 4.12 in our context. As we already explained, our $n$-graph $\mathcal{H}$ will have $V(\mathcal{H})=[m]^{2}$ and $E(\mathcal{H})=\left\{A \subset[m]^{2}:|A|=n, A \cong B\right\}$, that is, the vertices are the points in the grid and the edges are the $n$-sets isomorphic to $B$. Then an independent 
set is a set without a copy of $B$. It remains to check if $\mathcal{H}$ is a balanced $n$-graph and to find a family $\mathcal{F}$ such that $\mathcal{H}$ is $(\mathcal{F}, \varepsilon)$-dense. For the last one, we will use the following supersaturation result. For a configuration $A$, let $N_{B}(A)$ denote the number of copies of $B$ inside $A$.

Theorem 4.13. For a configuration $B$ of $n$ points and $\delta \in(0,1)$, there exists $\varepsilon>0$ such that for every $m$ the following holds. If $X \subset[m]^{2}$ with $|X| \geq \delta m^{2}$, then $N_{B}(X) \geq \varepsilon m^{2 n}$. Moreover, we can take $\varepsilon=\delta^{n}(3 \operatorname{gr}(B))^{-5 n^{2} \log (8 / \delta)}$.

Proof. The proof here is similar to the one of Theorem 3.16. Let $d=\lceil n \log (8 / \delta)\rceil$ and consider a configuration $A^{\prime} \in \bigotimes_{i=1}^{d} B$ as in Theorem 3.15, that is, a configuration $A^{\prime}$ such that $\operatorname{gr}\left(A^{\prime}\right) \leq 8^{d-1} \operatorname{gr}(B)^{2 d-1}$ and such that every $\frac{1}{2 \operatorname{gr}(B) \sqrt{2}}$-perturbation of $A^{\prime}$ is in $\bigotimes_{i=1}^{d} B$.

For an integer $k>0$, consider a homothety that dilates $A^{\prime}$ to a copy $A^{\prime \prime}$ which is $k$ times greater than $A^{\prime}$. The copy $A^{\prime \prime}$ can be embedded in $\left[k 8^{d-1} \operatorname{gr}(B)^{2 d-1}\right]^{2}$. Write $A^{\prime \prime}=\left\{a_{1}, \ldots, a_{n^{d}}\right\}$ and for every $a_{i}$, let $A_{i}$ be the points of $\left[k 8^{d-1} \operatorname{gr}(B)^{2 d-1}\right]^{2}$ that are inside the open ball centered at $a_{i}$ of radius $\frac{k}{2 \operatorname{gr}(B) \sqrt{2}}$. Consider the set $A=A_{1} \cup \cdots \cup A_{n^{d}}$. By the construction of $A$, every transversal is a configuration in $\bigotimes_{i=1}^{d} B$. Suppose that $k 8^{d-1} \operatorname{gr}(B)^{2 d-1} \leq m$, then $A \in[m]^{2}$. Therefore, we can apply Lemma 3.12 in the $\delta$ proportion $X$ of $[m]^{2}$ and obtain a translation of $A$ such that $X$ contains a $\frac{\delta}{4}$-proportion of it. Suppose without loss of generality that this translation is $A$.

We now prove that a $\frac{\delta}{4}$-proportion of $A$ contains many copies of $B$. Since $|X \cap A| \geq \frac{\delta}{4}|A|$, Lemma 3.7 says that there exists a set of indices $I$ of size at least $\frac{\delta n^{d}}{8}$ such that $\left|X \cap A_{i}\right| \geq$ $\frac{\delta}{8}\left|A_{i}\right|$ for every $i \in I$. Note that by Lemma 3.3 and our choice of $d$, we have that $Y \rightarrow_{\delta / 8} B$ for every $Y \in \bigotimes_{i=1}^{d} B$. Moreover, a copy of $B$ obtained by this lemma is a transversal of a configuration $Y^{\prime} \in \bigotimes_{i=1}^{d^{\prime}} B$ with $d^{\prime} \leq d$ and $Y^{\prime} \subset Y$. Consider the $\frac{\delta}{8}$-proportion of $A^{\prime \prime}$ given by choosing $a_{i} \in A^{\prime \prime}$ with $i \in I$ and let $J$ be the set of indices of the copy of $B$ inside this $\frac{\delta}{8}$-proportion of $A^{\prime \prime}$. We claim that any transversal of $\bigcup_{j \in J} A_{j}$ is isomorphic to $B$. This is because the set $\left\{a_{j}: j \in J\right\}$ is a transversal of a configuration $A^{\prime \prime \prime} \in \bigotimes_{i=1}^{d_{3}} B$ with $d_{3} \leq d$ and $A^{\prime \prime \prime} \subset A$ and because any $\frac{k}{2 \operatorname{gr}(B) \sqrt{2}}$-perturbation of $A^{\prime \prime}$ preserve the structure of a $\bigotimes_{i=1}^{d} B$.

Finally, using that $J \subset I$, we have that $\left|X \cap A_{j}\right| \geq \frac{\delta}{8}\left|A_{j}\right|$. By the same Pythagorean argument made in the proof of Lemma 3.14 , in the worst case, $A_{j}$ is a quarter of a circle and we have

$$
\left|A_{j}\right| \geq \frac{k^{2}}{16 \operatorname{gr}(B)^{2}}
$$

Therefore,

$$
N_{B}(X) \geq \prod_{j \in J}\left|X \cap A_{j}\right| \geq \prod_{j \in J} \frac{\delta}{8}\left|A_{j}\right| \geq \frac{\delta^{n} k^{2 n}}{16^{2 n} \operatorname{gr}(B)^{2 n}} .
$$


Taking $k=\left\lfloor\frac{m}{8^{d-1} \operatorname{gr}(B)^{2 d-1}}\right\rfloor$ we get,

$$
N_{B}(X) \geq \frac{\delta^{n}\left\lfloor\frac{m}{8^{d-1} \operatorname{gr}(B)^{2 d-1}}\right\rfloor^{2 n}}{16^{2 n} \operatorname{gr}(B)^{2 n}} \geq \frac{\delta^{n}}{(3 \operatorname{gr}(B))^{4 n d}} m^{2 n} \geq \frac{\delta^{n}}{(3 \operatorname{gr}(B))^{5 n^{2} \log (8 / \delta)}} m^{2 n} .
$$

Therefore $\varepsilon \leq \frac{\delta^{n}}{(3 \operatorname{gr}(B))^{5 n^{2} \log (8 / \delta)}}$ works.

Now we are able to prove the following version of the container theorem.

Theorem 4.14. Given a configuration $B$ with $n$ points and $\varepsilon>0$, there exist a constant $K:=K(B, \varepsilon)$ such that the following holds. For every integer $m \geq 2 \operatorname{gr}(B)$, let $\mathcal{I}=\{A \subset$ $\left.[m]^{2}: B \not \subset A\right\}$ be the family of subsets of $[m]^{2}$ without a copy of $B$. Then there exist families $\mathcal{C}$ and $\mathcal{S}$ of sets of $[m]^{2}$ and functions $f: \mathcal{I} \rightarrow \mathcal{S}$ and $g: \mathcal{S} \subset \mathcal{C}$ such that,

(i) $f(I) \leq K$, for every $I \in \mathcal{I}$.

(ii) $f(I) \subset I \subset g(f(I))$, for every $I \in \mathcal{I}$.

(iii) $|C|<\delta m^{2}$, for every $C \in \mathcal{C}$.

Where $\delta=8 \varepsilon^{\frac{1}{6 n^{2} \log (3 \operatorname{gr}(B))}}$ and the constant $K$ can be taken as $K=K_{1} \varepsilon^{-n} \log (1 / \varepsilon)$ for some constant $K_{1}:=K_{1}(B)$ depending only on $B$.

Proof. This is just an application of Theorem 4.12 with $\mathcal{H}$ as described before. Let $V(\mathcal{H})=$ $[m]^{2}$ and $E(\mathcal{H})=\left\{A \subset[m]^{2}: A \cong B\right\}$ the copies of $B$ inside $[m]^{2}$. Let $\mathcal{F}$ be the family of configurations $A \subset[m]^{2}$ with $|A| \geq \delta m^{2}$, where $\delta=8 \varepsilon^{\frac{1}{n^{2} \log (3 \operatorname{gr}(B))}}$. It is easy to see that $\mathcal{F}$ is increasing. By Theorem 4.13, we have that $e(\mathcal{H}[A])=N_{B}(A) \geq \delta^{n}(3 \operatorname{gr}(B))^{-5 n^{2} \log (8 / \delta)} \geq$ $\varepsilon m^{2 n} \geq \varepsilon e(\mathcal{H})$ and then $\mathcal{H}$ is $(\mathcal{F}, \varepsilon)$-dense.

It remains to prove that $\mathcal{H}$ is balanced. For this, we need a lower bound on $N_{B}\left([m]^{2}\right)$. As in Lemma 3.14, for an integer $k>0$, we can construct a copy $B^{\prime}$ of $B$ inside the $[k \cdot \operatorname{gr}(B)]^{2}$ grid such that every $\left(\frac{k}{2 \sqrt{2} \operatorname{gr}(B)}\right)$-perturbation is isomorphic to $B$. Let $B^{\prime}=\left\{b_{1}, \ldots, b_{n}\right\}$ and for every $b_{i} \in B^{\prime}$, let $B_{i}$ be the set of points in the $[k \cdot \operatorname{gr}(B)]^{2}$ that are inside the open ball centered at $b_{i}$ of radius $\frac{k}{2 \sqrt{2} \operatorname{gr}(B)}$. By the Lemma 2.10, we know that if $Y=\left\{y_{1}, \ldots, y_{n}\right\} \subset$ $[m]^{2}$ with $y_{i} \in B_{i}$, then $Y \cong B$. Therefore,

$$
N_{B}\left([k \cdot \operatorname{gr}(B)]^{2}\right) \geq \prod_{i=1}^{n}\left|B_{i}\right|
$$

In Lemma 3.14, we also saw that every set $B_{i}$ contains a grid of size $\frac{k}{4 \operatorname{gr}(B)}$, then $\left|B_{i}\right| \geq$ $\frac{k^{2}}{16 \operatorname{gr}(B)^{2}}$. All together, we have

$$
N_{B}\left([k \cdot \operatorname{gr}(B)]^{2}\right) \geq \frac{k^{2 n}}{4^{2 n} \operatorname{gr}(B)^{2 n}} .
$$

Taking $k=\left\lfloor\frac{m}{\operatorname{gr}(B)}\right\rfloor$, we obtain

$$
e(\mathcal{H})=N_{B}\left([m]^{2}\right) \geq \frac{\left\lfloor\frac{m}{\operatorname{gr}(B)}\right\rfloor^{2 n}}{4^{2 n} \operatorname{gr}(B)^{2 n}} \geq \frac{1}{(3 \operatorname{gr}(B))^{4 n}} m^{2 n} .
$$


The last estimate shows that $\mathcal{H}$ is a dense $n$-graph, i.e., $e(\mathcal{H}) \geq \alpha v(\mathcal{H})^{n}$ for some $\alpha>0$. This suffices to prove that $\mathcal{H}$ is balanced. Indeed, for any $1 \leq k \leq n$, we have

$$
\Delta_{k}(\mathcal{H}) \leq\left(\begin{array}{c}
v(\mathcal{H})-k \\
n-k
\end{array}\right) \leq v(\mathcal{H})^{n-k} \leq m^{2(n-k)}
$$

Choosing $p=\frac{1}{m^{2}}$ and $c=(3 \operatorname{gr}(B))^{4 n}$,

$$
\Delta_{k}(\mathcal{H}) \leq m^{2(n-k)}=c p^{k-1} \frac{m^{2(n-1)}}{(3 \operatorname{gr}(B))^{4 n}} \leq c p^{k-1} \frac{e(\mathcal{H})}{v(\mathcal{H})}
$$

Thus, applying Theorem 4.12 for $c=(3 \operatorname{gr}(B))^{4 n}$ and $\varepsilon>0$, we obtain a constant $K$ a family $\mathcal{S}$ and functions $f: \mathcal{I} \rightarrow \mathcal{C}, g: \mathcal{S} \rightarrow \overline{\mathcal{F}}$ such that,

(i) $f(I) \leq K p v(\mathcal{H})=K$, for every $I \in \mathcal{I}$.

(ii) $f(I) \subset I \subset g(f(I))$, for every $I \in \mathcal{I}$.

Take $\mathcal{C}=\overline{\mathcal{F}}$. By the definition of $\mathcal{F}$, we have that $|C| \leq \delta m^{2}$ for every $C \in \mathcal{C}$. Finally, in Theorem 4.12, the constant $K$ can be taken as $K=K^{\prime} \varepsilon^{-n} \log (1 / \varepsilon)$, where $K_{1}:=K_{1}(c, n)$ is a constant that depends only on $c$ and $n$. However, because $c=(3 \operatorname{gr}(B))^{4 n}$, we can view $K_{1}:=K_{1}(B)$.

\subsubsection{Counting theorem and proof of Theorem 1.4}

With the technical machinery of the container method, we are able to count the number of independent sets of a fixed size.

Theorem 4.15. For $\alpha \in(0,1$ ( and a configuration $B$ with $n$ points, there exists an integer $M:=M(B, \alpha)$ such that the following holds. For every sufficiently large $m$ and $N \geq M$ the number of $N$-subsets of $[\mathrm{m}]^{2}$ that do not contain a copy of $B$ is at most

$$
\left(\begin{array}{c}
\alpha m^{2} \\
N
\end{array}\right)
$$

Proof. Choose $\varepsilon=(\alpha / 800)^{6 n^{2} \log (3 \operatorname{gr}(B))}$, then $\delta=8 \varepsilon^{\frac{1}{6 n^{2} \log (3 \operatorname{gr}(B))}}=\alpha / 100$. Applying Theorem 4.14, we obtain a constant $K:=K(B, \varepsilon)$, families $\mathcal{S}, \mathcal{F}$ and functions $f: \mathcal{I} \rightarrow \mathcal{S}$, $g: \mathcal{I} \rightarrow \mathcal{C}$ satisfying items $(i),($ ii $)$ and $($ iii $)$. Set $M=\max \left\{\left\lceil\frac{K}{\delta}\right\rceil, 10 \log (1 / \delta)\right\}$ and suppose $A \subset[m]^{2}$ with $|A|=N \geq M$. If $A$ does not contain a copy of $B$, then by Theorem 4.14, there exist sets $f(A) \in \mathcal{S}$ and $g(f(A)) \in \mathcal{C}$ such that $|f(A)| \leq K,|g(f(A))| \leq \delta m^{2}$ and $f(A) \subset A \subset g(f(A))$. One can count all such sets $A$ using the elements of $\mathcal{S}$. In fact, recalling that $\mathcal{N}_{m, N}(B)=\left\{A \subset[m]^{2}: B \not \subset A,|A|=N\right\}$, we have,

$$
\left|\mathcal{N}_{m, N}(B)\right| \leq \sum_{S \in \mathcal{S}}\left(\begin{array}{c}
|g(S)| \\
N-|S|
\end{array}\right) \leq \sum_{s \leq K}\left(\begin{array}{c}
m^{2} \\
s
\end{array}\right)\left(\begin{array}{c}
\delta m^{2} \\
N-s
\end{array}\right) \leq \sum_{s \leq K}\left(\frac{e m^{2}}{s}\right)^{s}\left(\frac{N}{\delta m^{2}-N}\right)^{s}\left(\begin{array}{c}
\delta m^{2} \\
N
\end{array}\right)
$$

where here we use that $\left(\begin{array}{l}n \\ k\end{array}\right) \leq\left(\frac{e n}{k}\right)^{k}$ and that $\left(\begin{array}{c}\delta m^{2} \\ N\end{array}\right)=\frac{\left(\delta m^{2}-N+s\right) \ldots\left(\delta m^{2}-N+1\right)}{N(N-1) \ldots(N-s+1)}\left(\begin{array}{l}\delta m^{2} \\ N-s\end{array}\right) \geq$ $\left(\frac{\delta m^{2}-N}{N}\right)^{s}\left(\begin{array}{c}\delta m^{2} \\ N-s\end{array}\right)$. Noting that the function $x \mapsto(y / x)^{x}$ is increasing on $(0, y / e)$ and that for 
sufficiently large $m$ we have $\frac{\delta m^{2}}{2}>N$, it follows that

$$
\left|\mathcal{N}_{m, N}(B)\right| \leq \sum_{s \leq K}\left(\frac{2 e N}{\delta s}\right)^{s}\left(\begin{array}{c}
\delta m^{2} \\
N
\end{array}\right) \leq K\left(\frac{2 e N}{\delta K}\right)^{K}\left(\begin{array}{c}
\delta m^{2} \\
N
\end{array}\right)
$$

Using that $K \leq \delta N$ and that $\left(\begin{array}{c}\delta m^{2} \\ N\end{array}\right) \leq 2^{-N}\left(\begin{array}{c}\alpha m^{2} \\ N\end{array}\right)$ we have,

$$
\left|\mathcal{N}_{m, N}(B)\right| \leq\left(\frac{\delta N}{2^{N}}\right)\left(\frac{2 e}{\delta^{2}}\right)^{\delta N}\left(\begin{array}{c}
\alpha m^{2} \\
N
\end{array}\right)
$$

Because $\delta=\alpha / 100<1 / 100$, we have that $\frac{2 e}{\delta^{2}} \leq 2^{1 / 2 \delta}$ and then

$$
\left|\mathcal{N}_{m, N}(B)\right| \leq \frac{\delta N}{2^{N / 2}}\left(\begin{array}{c}
\alpha m^{2} \\
N
\end{array}\right) \leq\left(\begin{array}{c}
\alpha m^{2} \\
N
\end{array}\right)
$$

which is true for $N \geq 10 \log (1 / \delta)$.

To prove Theorem 1.4 we perform the same trick used in the extremal number problem. For a fixed $N$ we pick an appropriate $\alpha$ such that $M(B, \alpha) \leq N$. Here it is important to know the behavior of $K$ as a function of $\varepsilon$.

Proof of Theorem 1.4. Set $\alpha=N^{-c}$, where $c:=c(B)=\frac{1}{10 n^{3} \log (3 \operatorname{gr}(B))}$. For this value of $\alpha$, let $\varepsilon=(\alpha / 800)^{6 n^{2} \log (3 \operatorname{gr}(B))}$ and $\delta=\alpha / 100$. Also let $K:=K(B, \varepsilon)$ and $M:=M(B, \alpha)$ be the constants obtained by applying Theorems $4.14,4.15$ for parameters $B$, $\varepsilon$ and $B, \alpha$, respectively. An easy computation using that $K=K_{1} \varepsilon^{-n} \log (1 / \varepsilon)$ shows that,

$$
\begin{aligned}
& M(B, \alpha)=\max \left\{\left\lceil\frac{2 K(B, \varepsilon)}{\delta}\right\rceil, 100 \log (1 / \delta)\right\} \leq 3 K_{1} \delta^{-1} \varepsilon^{-n} \log (1 / \varepsilon) \leq 3 K_{1} \delta^{-1} \varepsilon^{-(n+1)} \\
& \leq K_{2}\left(\frac{16}{\alpha}\right)^{6 n^{2}(n+1) \log (3 \operatorname{gr}(B))+1} \leq K_{3}\left(\frac{1}{\alpha}\right)^{8 n^{3} \log (3 \operatorname{gr}(B))}=K_{3} N^{4 / 5} \leq N
\end{aligned}
$$

for sufficiently large $N$ and constants $K_{1}, K_{2}, K_{3}$ depending only on $B$. Therefore, by Theorem 4.15 we have that,

$$
\left|\mathcal{N}_{m, N}(B)\right| \leq\left(\begin{array}{c}
\alpha m^{2} \\
N
\end{array}\right) \leq \frac{\alpha^{N} m^{2 N}}{N !}
$$

and by Proposition 4.10,

$$
\mathbb{P}(B \not \subset U)=\lim _{m \rightarrow \infty} \frac{N !\left|\mathcal{N}_{m, N}(B)\right|}{m^{2 N}} \leq \alpha^{N}=\left(\frac{1}{N}\right)^{c N} .
$$

We conclude this section with an application of the last theorem. We will compute the probability of an $N$-random set $U$ being in a certain hereditary property. A hereditary property of order types is a family $\mathcal{P}$ of order types satisfying the condition that if $X \in \mathcal{P}$ and $Y \subset X$, then $Y \in \mathcal{P}$, that is, a hereditary property is a decreasing family. 
Given a configuration $X$, define

$$
\operatorname{Forb}(X)=\{A: X \not \subset A\}
$$

as the family of order types that do not contain a copy of $X$. For a family $\mathcal{F}$ of order types we define $\operatorname{Forb}(\mathcal{F})$ as the family of order types that do not contain a copy of any element of $\mathcal{F}$.

It is not difficult to see that every hereditary property $\mathcal{P}$ can be defined as $\operatorname{Forb}(\mathcal{F})$ for some family $\mathcal{F}$. For instance, the family $C O N V$ of convex sets is a hereditary property. This is because any subset of a convex set is also convex. One can see, that another way to formulate this family is by considering the family $\operatorname{Forb}(T)$, where $T$ is the triangle with an interior point. Because any convex set does not contain interior point, we have that $C O N V \subset \operatorname{Forb}(T)$. On the other hand, if the convex hull of a configuration in $\operatorname{Forb}(T)$ contains an interior point, then a triangulation generates $T$. Therefore, $C O N V=\operatorname{Forb}(T)$.

Given a configuration $B$ of $n$ points and a random set $U$ of $N$ points inside the unit square, the problem studied in this section can be translated in the new notation, i.e., to estimate bounds for the probability of $U \in \operatorname{Forb}(B)$. However, because

$$
\mathbb{P}\left(U \in \operatorname{Forb}\left(\mathcal{F}_{1}\right)\right) \leq \mathbb{P}\left(U \in \operatorname{Forb}\left(\mathcal{F}_{2}\right)\right)
$$

if $\mathcal{F}_{2} \subset \mathcal{F}_{1}$, in order to obtain an upper bound to the probability of $\mathbb{P}(U \in \operatorname{Forb}(\mathcal{F}))$ we just need to solve the case where $\mathcal{F}$ contains only one element. This observation gives the following corollary.

Corollary 4.16. Let $\mathcal{P}$ be an infinite hereditary property of order types and let $U$ be the set obtained by choosing at random $N$ points inside the unit square. Then, there exists constants $c_{1}, c_{2}$ depending only on $\mathcal{P}$ such that,

$$
\left(\frac{1}{N}\right)^{c_{1} N} \leq \mathbb{P}(U \in \mathcal{P}) \leq\left(\frac{1}{N}\right)^{c_{2} N}
$$

Proof. Let $\mathcal{F}$ be the family such that $\mathcal{P}=\operatorname{Forb}(\mathcal{F})$. Suppose that $\mathcal{F}$ contains a convex set $C$ of $m$ points. Then by Theorem 3.4, every configuration $A$ with at least $\operatorname{ES}(m)+1$ points contains a copy of $C$. This implies that every configuration in $\mathcal{P}$ contains at most $\mathrm{ES}(m)$ points and we have that $|\mathcal{P}|$ is finite, a contradiction. Therefore, $\mathcal{F}$ does not contain a convex set. An immediate consequence is that every convex set is an element of $\mathcal{P}$. Thus, by Theorem 4.1,

$$
\mathbb{P}(U \in \mathcal{P}) \geq \mathbb{P}(U \text { is convex })=\left(\frac{\left(\begin{array}{c}
2 N-2 \\
N-1
\end{array}\right)}{N !}\right)^{2} \geq\left(\frac{1}{N}\right)^{c_{1} N}
$$

for an appropriate constant $c_{1}>0$. 
For the upper bound, just consider a configuration $B \in \mathcal{F}$. Then, by Theorem 1.4,

$$
\mathbb{P}(U \in \mathcal{P}) \leq \mathbb{P}(U \in \operatorname{Forb}(B)) \leq\left(\frac{1}{N}\right)^{c_{2} N},
$$

with $c_{2}:=c_{2}(B)$ a constant depending on $\mathcal{P}$. 


\section{Chapter 5}

\section{Final Remarks}

The combinatorial aspect of order types is a very recent field in combinatorics. Therefore, there are many unexplored territories and open problems. In this chapter we propose some problems related with our work.

In Section 3.1, we studed the problem of finding bounds for $f(B, \alpha)$ for a fixed configuration $B$ and a real number $\alpha \in(0,1)$. Our approach is based on iterating blow-ups of $B$ until we obtain an $\alpha$-proprotion whit the desired copy. This process only uses the fact that order types are open, which suggests room to improvement.

Problem 5.1. Determine whether $f(n, \alpha)$ is polynomial or exponential on $n$ and $\alpha$.

One simple case that we do not know an efficient method to compute $f(B, \alpha)$ is when $B$ is a pentagon with a center point. It would be interesting to find other bounds for this configuration that avoid the iterative blow-up method.

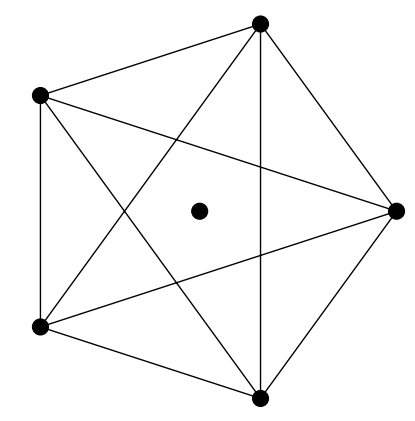

Figure 5.1: Pentagon with a center point

The following problem was proposed by Hao Huang and is based on its analogue in graph theory.

Problem 5.2. Given an order type $B$ of $n$ points and a integer $N>0$. Find the maximum number of copies of $B$ inside a configuration of $N$ points.

In Section 3.2 we developed a method to give upper bounds for $\operatorname{ex}(N, B)$. This method can give relatively sharp bounds as we can see in Theorem 3.19. However, we do not 
have a good lower bound, that is, a lower bound that in fact uses some properties of our configuration $B$. It would be interesting to find better lower bounds.

Problem 5.3. Does there exists a configuration $B$ such that $\operatorname{ex}(N, B)$ is not linear on $N$ ?

We also conjecture that the behavior of the convex case should be as follows.

Conjecture 5.4. If $B$ is a convex set of $n$ points, then $\operatorname{ex}(N, B)=\Theta\left(n^{3 / 2} N\right)$.

At last, in Chapter 4 we studied a specific random space and obtain some results on probabilities of order types and hereditary properties. All of these were motivated by the following question proposed by Yoshiharu Kohayakawa.

Problem 5.5. Let $R(n)$ be the minimum integer $N$ such that there exists a configuration $n$-universal of $N$ points. Determine bounds for $R(n)$.

An obvious application of Theorem 2.3 gives us that $R(n) \leq 2^{4 n \log n+O(n)}$. For the lower bound, we use the simple counting argument that the number of $n$-subsets of an $n$-universal is at least $2^{4 n \log n+O(n)}$. Thus,

$$
\left(\begin{array}{c}
R(n) \\
n
\end{array}\right) \geq 2^{4 n \log n+O(n)}
$$

and we obtain that

$$
R(n) \geq n^{5+o(1)}
$$

Clearly, there is a huge gap between these two bounds. Theorem 1.3 indicates that is probably not helpful to use our random process to obtain small $n$-universal order types. Despite of that, it would be interesting to know more about the probabilities in this space. We finish this chapter by conjecturing that the probability of an order type is strongly related to its $g r$ parameter.

Conjecture 5.6. There exist constants $c_{1}, c_{2}$ such that for every sufficiently large $n$ the following holds. Let $B$ be an order type of $n$ points and $U$ be the set obtained by choosing $n$ random points inside the unit square, then

$$
(\operatorname{gr}(B))^{-c_{1} n} \leq \mathbb{P}(U \cong B) \leq(\operatorname{gr}(B))^{-c_{2} n} .
$$

Theorem 4.1 states that this is true for convex sets. We also know, by Theorem 1.4, that this is true for configurations with polynomial grid size and without containing a small order type. This is the case for the double circle [3]. 


\section{Appendix A}

\section{Asymptotic Estimates}

In this appendix we show an important estimate number theoretic estimate for our work (Theorem A.2). The estimate relies on the following result known as Abel's summation formula.

Theorem A.1 ([1], Theorem 4.2). Let $\left\{a_{n}\right\}_{n \geq 1}$ be a sequence of real numbers and let $f:[1, \infty] \rightarrow \mathbb{R}$. For each real number $x \geq 1$, let

$$
A(x)=\sum_{n \leq x} a_{n}
$$

and assume that $f(x)$ has a continuous derivative for $x \geq 1$. Then

$$
\sum_{n \leq x} a_{n} f(n)=A(x) f(x)-\int_{1}^{x} A(t) f^{\prime}(t) \mathrm{d} t
$$

The next theorem involves some estimates over the sums of euler totient functions. Note that the euler totient function $\varphi$ is defined by $\varphi(n)=|\{j \in[n]: \operatorname{gcd}(n, j)=1\}|$, i.e., the number of integers in $[n]$ which coprime with $n$.

Theorem A.2. The estimates

$$
\begin{aligned}
\sum_{n \leq x} \varphi(n) & =\frac{3}{\pi^{2}} x^{2}+O(x \log x), \\
\sum_{n \leq x} n \varphi(n) & =\frac{2}{\pi^{2}} x^{3}+O\left(x^{2} \log x\right)
\end{aligned}
$$

hold for all $x$.

The proof of this theorem requires a standard result in number theory related with the Möbius function. The Möbius function $\mu$ is defined as follows. For every integer $n>1$, 
write $n=p_{1}^{\alpha_{1}} \ldots p_{k}^{\alpha_{k}}$ as its prime factorization. Then

$$
\mu(n)= \begin{cases}1, & \text { if } n=1 \\ (-1)^{k}, & \text { if } \alpha_{1}=\cdots=\alpha_{k}=1 \\ 0, & \text { otherwise }\end{cases}
$$

Notice that $\mu(n)=0$ if and only if $n$ has a squared factor.

Theorem A.3 ([1], Theorem 2.3). If $n \geq 1$ we have

$$
\varphi(n)=\sum_{d \mid n} \mu(d) \frac{n}{d}
$$

Now using Abel's summation formula we are able to prove this theorem.

Proof of Theorem A.2. First we want to estimate $\sum_{n \leq x} \frac{\varphi(n)}{n}$. Theorem A.3 gives us

$$
\frac{\varphi(n)}{n}=\sum_{d \mid n} \frac{\mu(d)}{d} .
$$

Summing for all $n \leq x$,

$$
\begin{aligned}
& \sum_{n \leq x} \frac{\varphi(n)}{n}=\sum_{n \leq x} \sum_{d \mid n} \frac{\mu(d)}{d}=\sum_{d \leq x} \frac{\mu(d)}{d} \sum_{\substack{n \leq x \\
d \mid n}} 1=\sum_{d \leq x} \frac{\mu(d)}{d}\left(\frac{x}{d}+O(1)\right)= \\
& =x \sum_{d \leq x} \frac{\mu(d)}{d^{2}}+O\left(\sum_{d \leq x} \frac{1}{d}\right)=x\left(\sum_{d=1}^{\infty} \frac{\mu(d)}{d^{2}}-\sum_{d>x} \frac{\mu(d)}{d^{2}}\right)+O\left(\sum_{d \leq x} \frac{1}{d}\right) \\
& =x \sum_{d=1}^{\infty} \frac{\mu(d)}{d^{2}}+O\left(x\left(\sum_{d>x} \frac{1}{d^{2}}\right)+\sum_{d \leq x} \frac{1}{d}\right) .
\end{aligned}
$$

Standard estimates gives that

$$
\begin{aligned}
& \sum_{d \leq x} \frac{1}{d}=O(\log x), \\
& \sum_{d>x} \frac{1}{d^{2}} \leq \int_{x-1}^{\infty} \frac{1}{t^{2}} \mathrm{~d} t=\frac{1}{x-1},
\end{aligned}
$$

and one can estimate $\sum_{d=1}^{\infty} \frac{\mu(d)}{d^{2}}$ by

$$
\begin{aligned}
& \sum_{d=1}^{\infty} \frac{\mu(d)}{d^{2}}=1+\sum_{p_{1}, \ldots, p_{k}} \frac{(-1)^{k}}{p_{1}^{2} \ldots p_{k}^{2}}=\prod_{p}\left(1-\frac{1}{p^{2}}\right)=\prod_{p}\left(\frac{1}{1-\frac{1}{p^{2}}}\right)^{-1}= \\
& \prod_{p}\left(1+\frac{1}{p^{2}}+\frac{1}{p^{4}}+\cdots+\right)^{-1}=\left(\sum_{n=1}^{\infty} \frac{1}{n^{2}}\right)^{-1}=\frac{6}{\pi^{2}},
\end{aligned}
$$


where we use that $\sum_{n=1}^{\infty} \frac{1}{n^{2}}=\frac{\pi^{2}}{6}$. All together give

$$
\sum_{n \leq x} \frac{\varphi(n)}{n}=\frac{6}{\pi^{2}} x+O(\log x) .
$$

To get the desired estimate we now apply the Abel summation formula twice. First with $a_{n}=\frac{\varphi(n)}{n}$ and $f(t)=t$. Noting that $A(x)=\sum_{n \leq x} \frac{\varphi(n)}{n}$, we obtain

$$
\begin{aligned}
& \sum_{n \leq x} \varphi(n)=\sum_{n \leq x} a_{n} f(n)=A(x) f(x)-\int_{1}^{x} A(t) f^{\prime}(t) \mathrm{d} t \\
& =\left(\frac{6}{\pi^{2}} x+O(\log x)\right) x-\int_{1}^{x}\left(\frac{6}{\pi^{2}} t+O(\log t)\right) \mathrm{d} t \\
& =\frac{6}{\pi^{2}} x^{2}+O(x \log x)-\frac{6}{\pi^{2}} \int_{1}^{x} t \mathrm{~d} t+O\left(\int_{1}^{x} \log t \mathrm{~d} t\right) \\
& =\frac{6}{\pi^{2}} x^{2}+O(x \log x)-\frac{3}{\pi^{2}} x^{2}+O(x \log x)=\frac{3}{\pi^{2}} x^{2}+O(x \log x) .
\end{aligned}
$$

Then we apply again with $a_{n}=\frac{\varphi(n)}{n}$ and $f(t)=t^{2}$,

$$
\begin{aligned}
& \sum_{n \leq x} n \varphi(n)=\sum_{n \leq x} a_{n} f(n)=A(x) f(x)-\int_{1}^{x} A(t) f^{\prime}(t) \mathrm{d} t \\
& =\left(\frac{6}{\pi^{2}} x+O(\log x)\right) x^{2}-\int_{1}^{x} 2 t\left(\frac{6}{\pi^{2}} t+O(\log t)\right) \mathrm{d} t \\
& =\frac{6}{\pi^{2}} x^{3}+O\left(x^{2} \log x\right)-\frac{12}{\pi^{2}} \int_{1}^{x} t^{2} \mathrm{~d} t+O\left(\int_{1}^{x} t \log t \mathrm{~d} t\right) \\
& =\frac{6}{\pi^{2}} x^{3}+O\left(x^{2} \log x\right)-\frac{4}{\pi^{2}} x^{3}+O\left(x^{2} \log x\right)=\frac{2}{\pi^{2}} x^{3}+O\left(x^{2} \log x\right) .
\end{aligned}
$$




\section{Bibliography}

[1] T. Apostol. Introduction to analytic number theory. Springer-Verlag, 1976. A.1, A.3

[2] József Balogh, Robert Morris, and Wojciech Samotij. Independent sets in hypergraphs. J. Amer. Math. Soc., 28(3):669-709, 2015. 4.2, 4.2.2, 4.12

[3] S. Bereg, R. Fabila-Monroy, D. Flores-Peñaloza, M. A. Lopez, and P. Pérez-Lantero. Embedding the double circle in a square grid of minimum size. Internat. J. Comput. Geom. Appl., 24(3):247-258, 2014. 5

[4] Robert G. Bland and Michel Las Vergnas. Orientability of matroids. J. Combinatorial Theory Ser. B., 24(1):94-123, 1978. 1

[5] Jürgen Bokowski and Bernd Sturmfels. Computational synthetic geometry, volume 1355 of Lecture Notes in Mathematics. Springer-Verlag, Berlin, 1989. 1

[6] Jason I. Brown and Vojtěch Rödl. A Ramsey type problem concerning vertex colourings. J. Combin. Theory Ser. B, 52(1):45-52, 1991. 3.1, 3.1.2

[7] Xiaomin Chen, János Pach, Mario Szegedy, and Gábor Tardos. Delaunay graphs of point sets in the plane with respect to axis-parallel rectangles. Random Structures Algorithms, 34(1):11-23, 2009. 1

[8] D. Eppstein. Forbidden Configurations in Discrete Geometry. Cambridge University Press, 2018. 1

[9] P. Erdős and G. Szekeres. A combinatorial problem in geometry. Compos. Math. 2, pages $463-470,1935.1,3.1 .2$

[10] P. Erdős and G. Szekeres. On some extremum problems in elementary geometry. Ann. Univ. Sci. Budapest. Eötvös Sect. Math. 3-4, pages 53-62, 1961. 3.1.2

[11] P. Erdös and A. H. Stone. On the structure of linear graphs. Bull. Amer. Math. Soc., 52:1087-1091, 1946. 3

[12] H. Furstenberg and Y. Katznelson. An ergodic Szemerédi theorem for commuting transformations. J. Analyse Math., 34:275-291 (1979), 1978. 3.11, 3.2 
[13] J. E. Goodman, R. Pollack, and B. Sturmfels. Coordinate representation of order types requires exponential storage. In Proceedings of the Twenty-first Annual ACM Symposium on Theory of Computing, STOC '89, pages 405-410, New York, NY, USA, 1989. ACM. 2.3, 2.18

[14] Jacob E. Goodman and Richard Pollack. Multidimensional sorting. SIAM J. Comput., 12(3):484-507, 1983. 2.2

[15] Jacob E. Goodman and Richard Pollack. Upper bounds for configurations and polytopes in $\mathbf{R}^{d}$. Discrete Comput. Geom., 1(3):219-227, 1986. 2.1, 2.3

[16] W. T. Gowers. A new proof of Szemerédi's theorem. Geom. Funct. Anal., 11(3):465$588,2001.3 .2$

[17] W. T. Gowers. Hypergraph regularity and the multidimensional Szemerédi theorem. Ann. of Math. (2), 166(3):897-946, 2007. 3.11, 3.2

[18] D. Yu. Grigor ev and N. N. Vorobjov, Jr. Solving systems of polynomial inequalities in subexponential time. J. Symbolic Comput., 5(1-2):37-64, 1988. 2.3.2, 2.24

[19] B. Grünbaum. Arrangements and Spreads. Amer. Math. Soc., Providence, 1972. 2.3

[20] Beat Jaggi, Peter Mani-Levitska, Bernd Sturmfels, and Neil White. Uniform oriented matroids without the isotopy property. Discrete Comput. Geom., 4(2):97-100, 1989. $2.3 .1,2.3 .1$

[21] Vojtěch Jarník. Über die Gitterpunkte auf konvexen Kurven. Math. Z., 24(1):500-518, 1926. 3.2 .4

[22] Gyula Károlyi and József Solymosi. Erdos-Szekeres theorem with forbidden order types. J. Combin. Theory Ser. A, 113(3):455-465, 2006. 1, 3.1.3

[23] Gyula Károlyi and Géza Tóth. Erdos-Szekeres theorem for point sets with forbidden subconfigurations. Discrete Comput. Geom., 48(2):441-452, 2012. 1

[24] Brendan Nagle, Vojtěch Rödl, and Mathias Schacht. The counting lemma for regular k-uniform hypergraphs. Random Structures Algorithms, 28(2):113-179, 2006. 3.11, 3.2

[25] Jaroslav Nešetřil and Pavel Valtr. A Ramsey-type theorem in the plane. Combin. Probab. Comput., 3(1):127-135, 1994. 1

[26] Jaroslav Nešetřil and Pavel Valtr. A Ramsey property of order types. J. Combin. Theory Ser. A, 81(1):88-107, 1998. 1

[27] Jürgen Richter-Gebert and Günter M. Ziegler. Oriented matroids. In Handbook of discrete and computational geometry, CRC Press Ser. Discrete Math. Appl., pages 111132. CRC, Boca Raton, FL, 1997. 1

[28] Vojtěch Rödl and Jozef Skokan. Regularity lemma for $k$-uniform hypergraphs. Random Structures Algorithms, 25(1):1-42, 2004. 3.2 
[29] David Saxton and Andrew Thomason. Hypergraph containers. Invent. Math., 201(3):925-992, 2015. 4.2, 4.2.2

[30] A. Seidenberg. Lectures in projective geometry. Van Nostrand, 1962. 2.2.2

[31] M. Spivak. Calculus on Manifolds: A modern approach to classical theorems of advanced calculus. Addison-Wesley, 1965. 2.1.1, 2.6, 2.1.1

[32] F. W. Stevenson. Projective planes. Freeman, 1972. 2.2.2

[33] Andrew Suk. On the Erdos-Szekeres convex polygon problem. J. Amer. Math. Soc., 30(4):1047-1053, 2017. 3.1.2, 3.4

[34] P. Valtr. Probability that $n$ random points are in convex position. Discrete Comput. Geom., 13(3-4):637-643, 1995. 1, 4.1

[35] Neil L. White. A nonuniform matroid which violates the isotopy conjecture. Discrete Comput. Geom., 4(1):1-2, 1989. 2.2.2 